Supporting Information

\title{
Divergent Synthesis of Natural Benzyl Salicylate and Benzyl Gentisate Glucosides
}

Dariya D. Fedorova, ${ }^{\dagger}$ Dariya S. Nazarova, ${ }^{\dagger}$ David L. Avetyan, ${ }^{\dagger}{ }^{\ddagger}$ Andrey Shatskiy, ${ }^{\dagger, \S}$ Maxim L. Belyanin, ${ }^{\dagger}$ Markus D. Kärkäs, ${ }^{\S}$ and Elena V. Stepanova*,+,^

† Tomsk Polytechnic University, Lenin Avenue 30, Tomsk 634050, Russia

‡ Siberian State Medical University, Moskovskiy Trakt 2, Tomsk 634050, Russia

$\S$ Department of Chemistry, KTH Royal Institute of Technology, Stockholm 10044, Sweden

$\wedge$ N. D. Zelinsky Institute of Organic Chemistry of the Russian Academy of Sciences, Leninsky Prospect 47, Moscow 119991, Russia

E-mail: eline m@mail.ru, glycoside.m@gmail.com 


\section{Table of Contents}

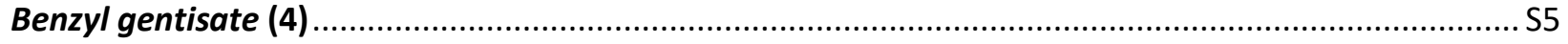

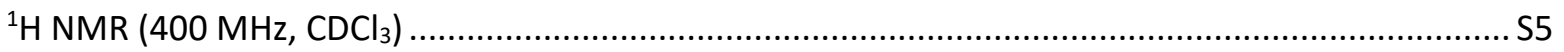

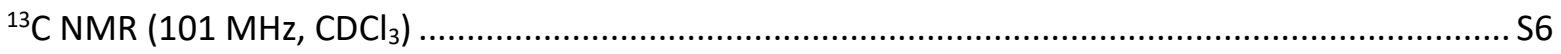

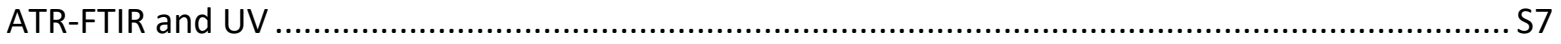

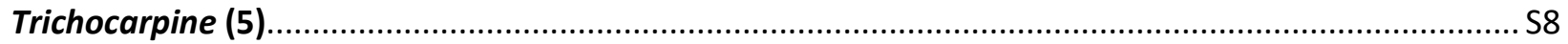

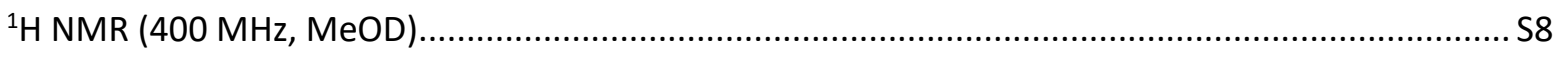

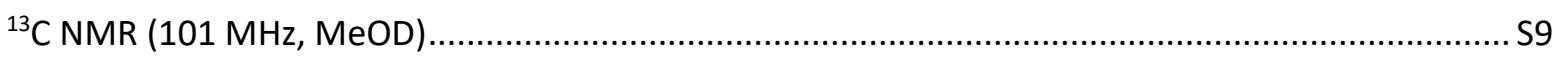

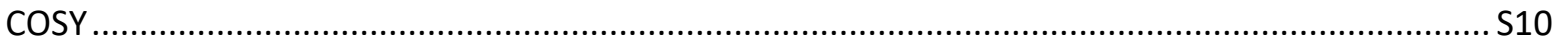

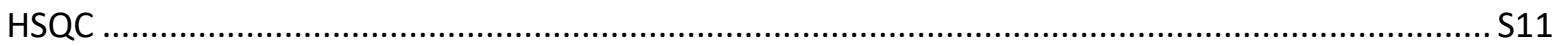

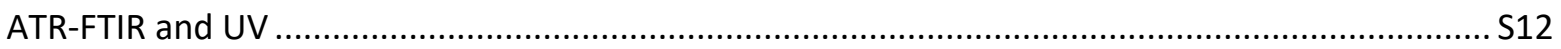

2-O-Acetyltrichocarpine (6) ......................................................................................................

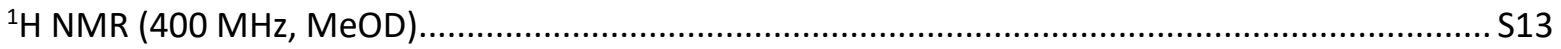

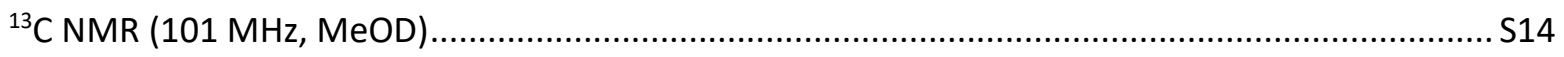

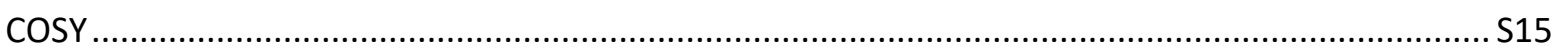

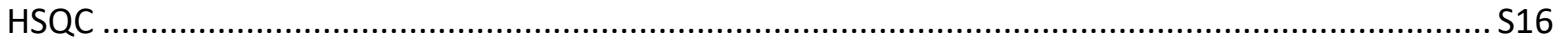

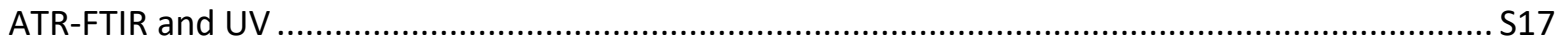

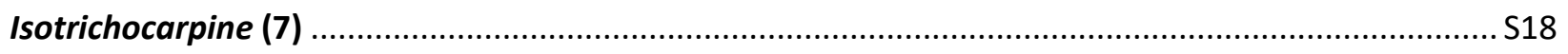

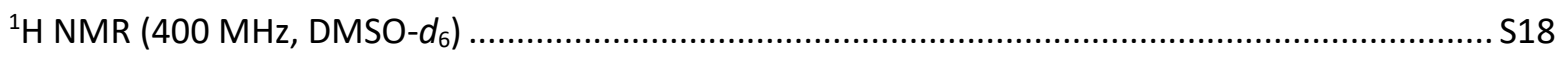

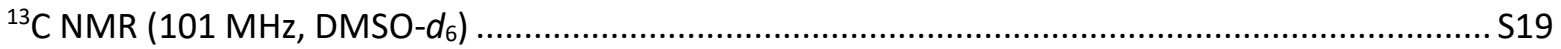

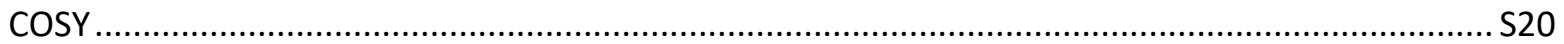

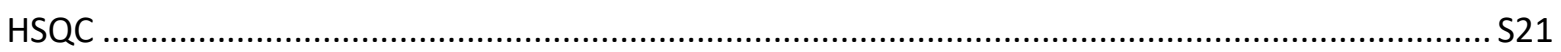

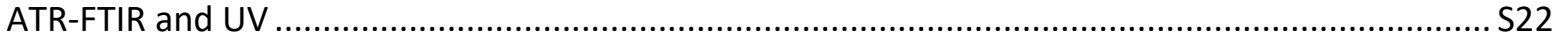

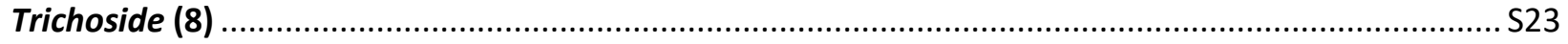

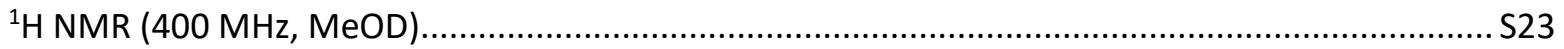

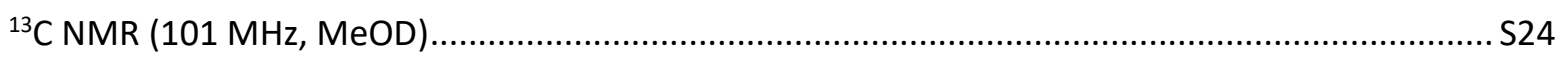

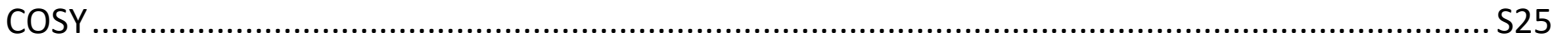

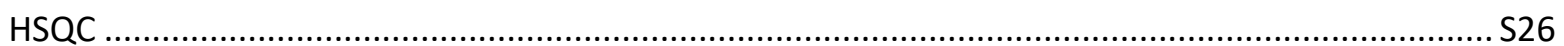

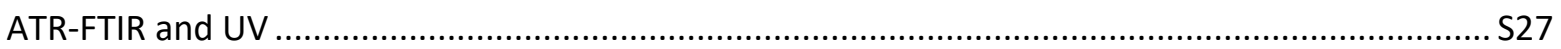

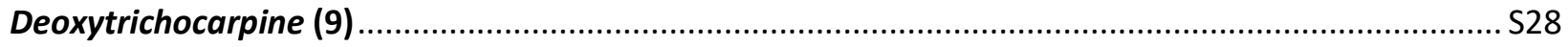

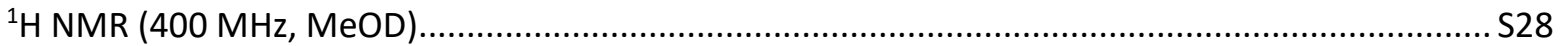

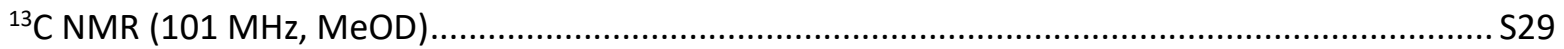

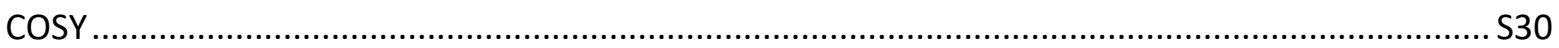


HSQC .

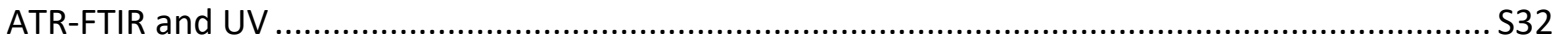

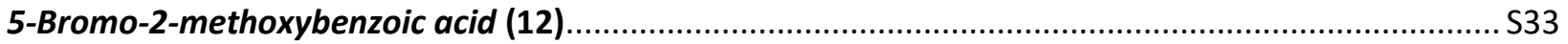

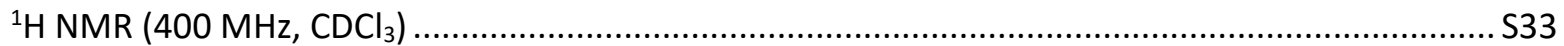

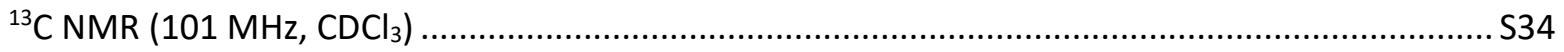

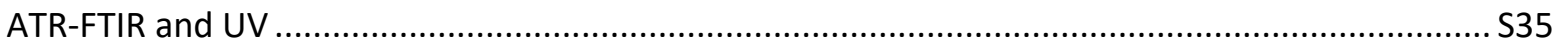

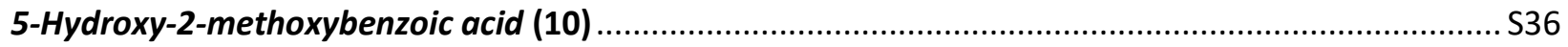

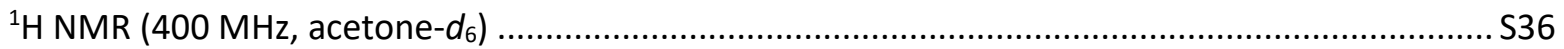

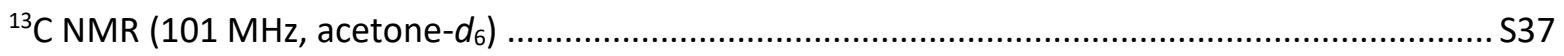

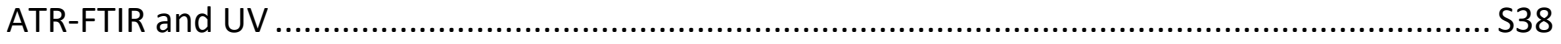

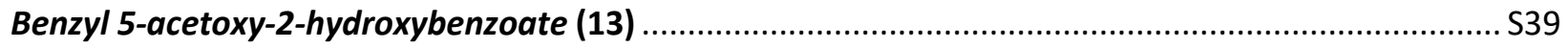

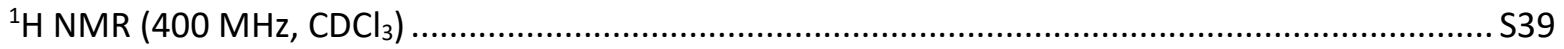

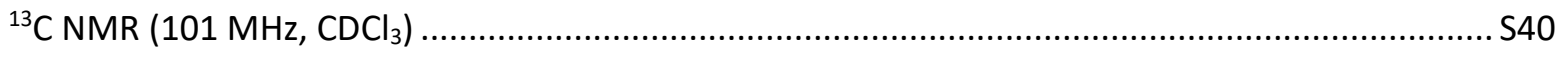

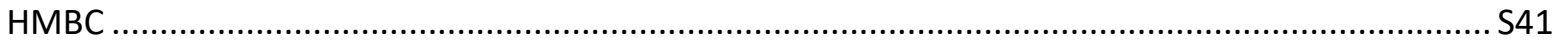

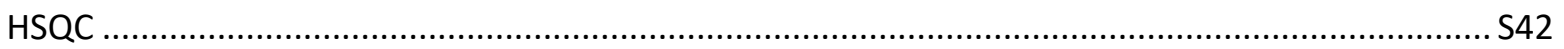

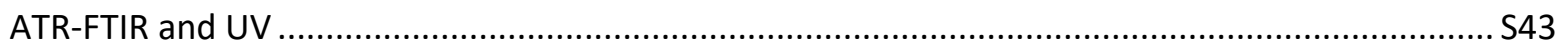

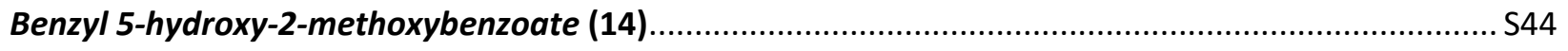

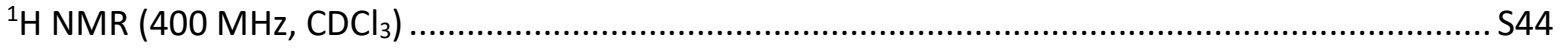

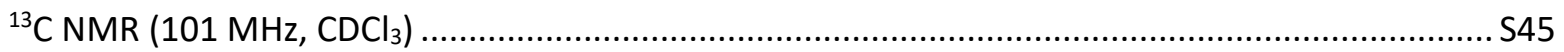

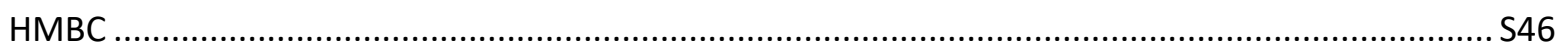

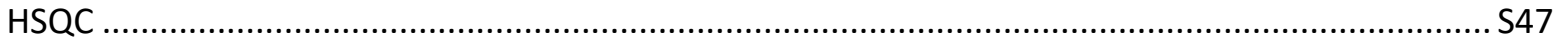

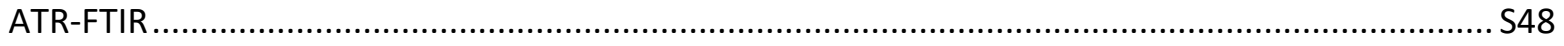

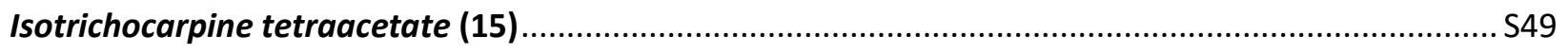

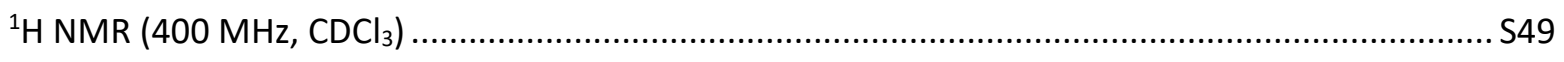

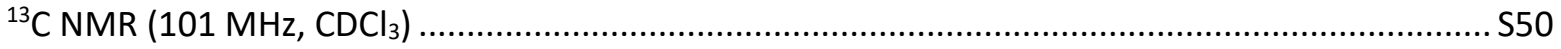

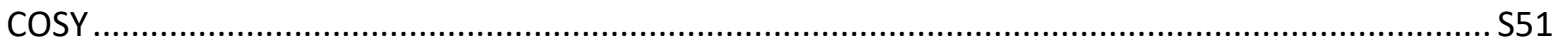

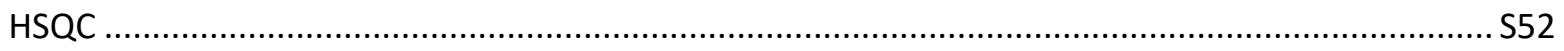

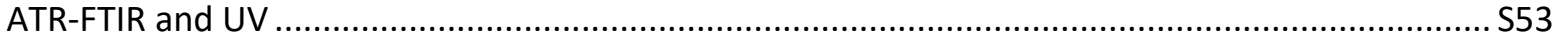

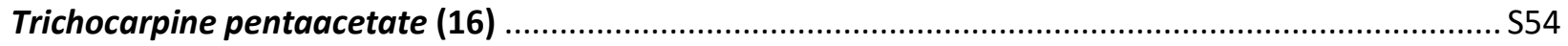

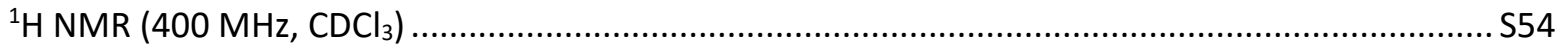

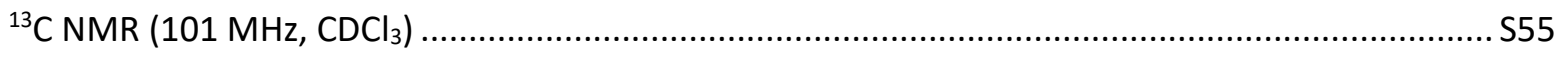

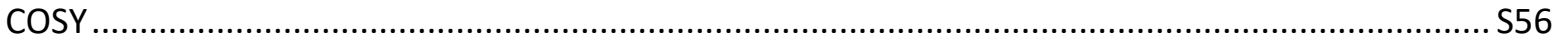

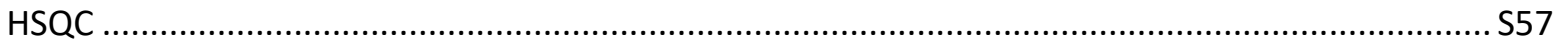




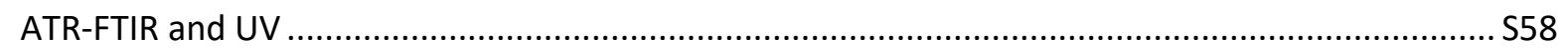

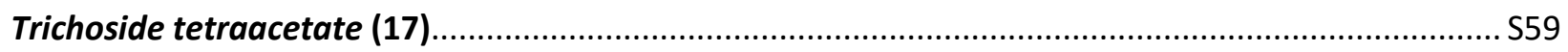

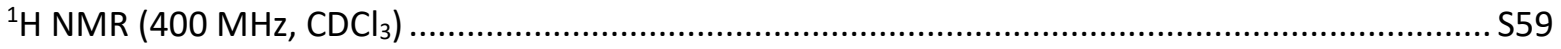

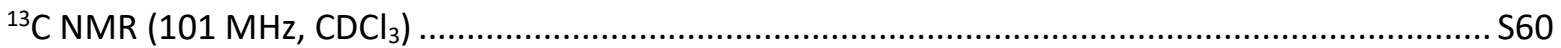

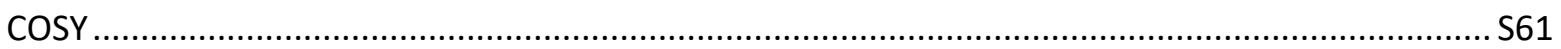

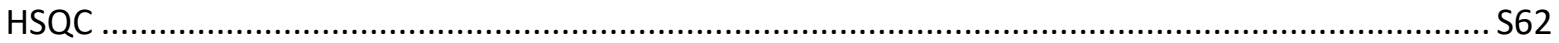

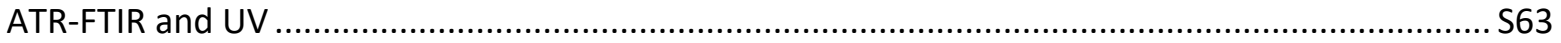

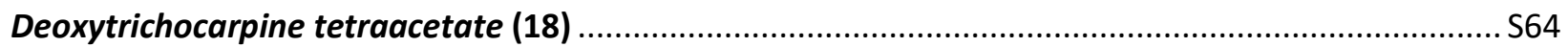

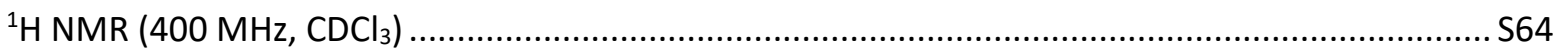

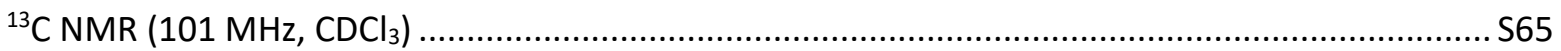

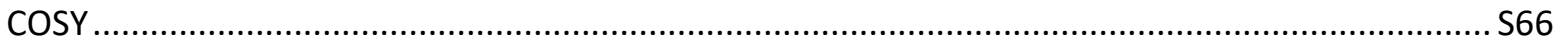

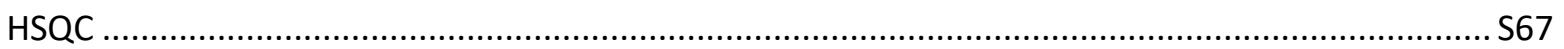

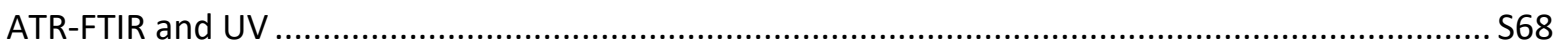

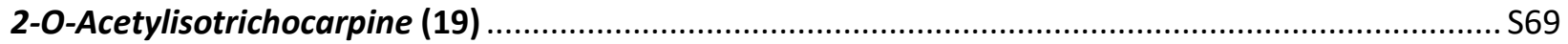

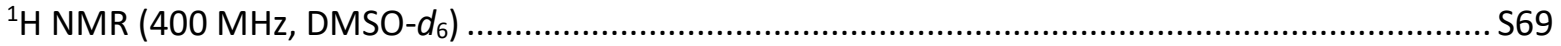

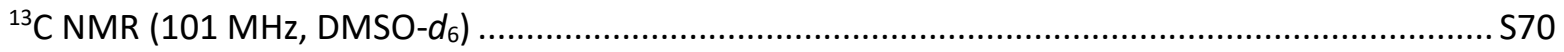

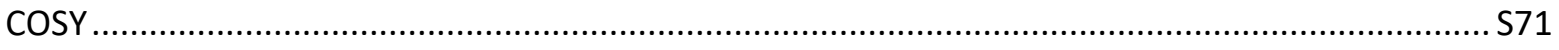

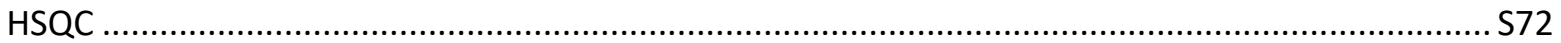

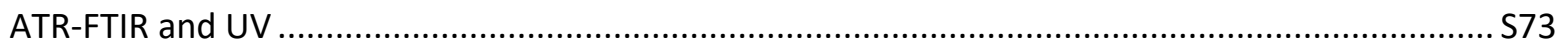

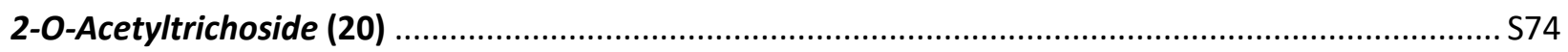

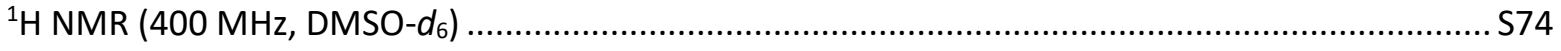

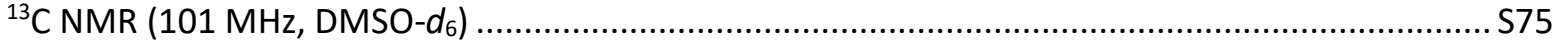

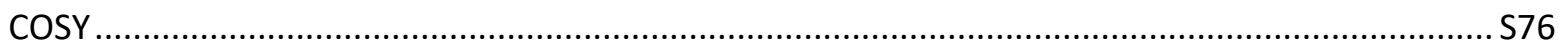

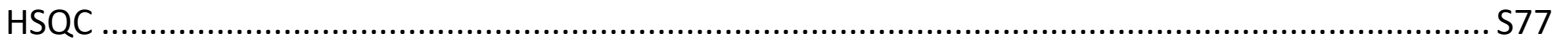

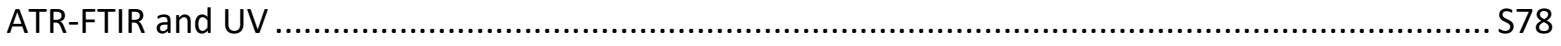

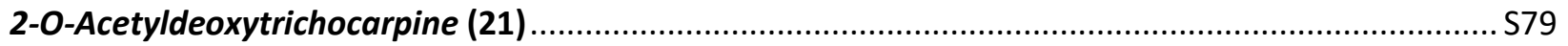

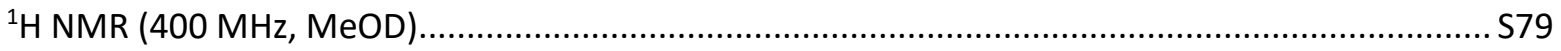

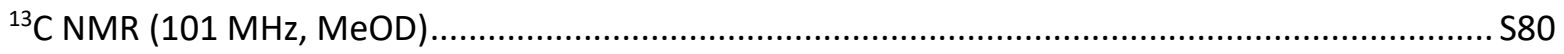

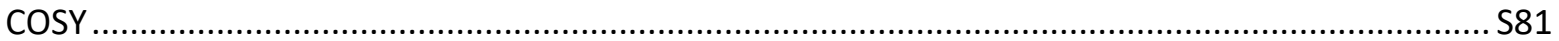

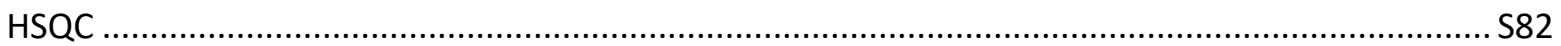

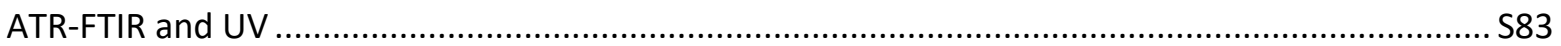




\section{Benzyl gentisate (4)}

${ }^{1} \mathrm{H}$ NMR (400 MHz, $\mathrm{CDCl}_{3}$ ) of benzyl gentisate (4)

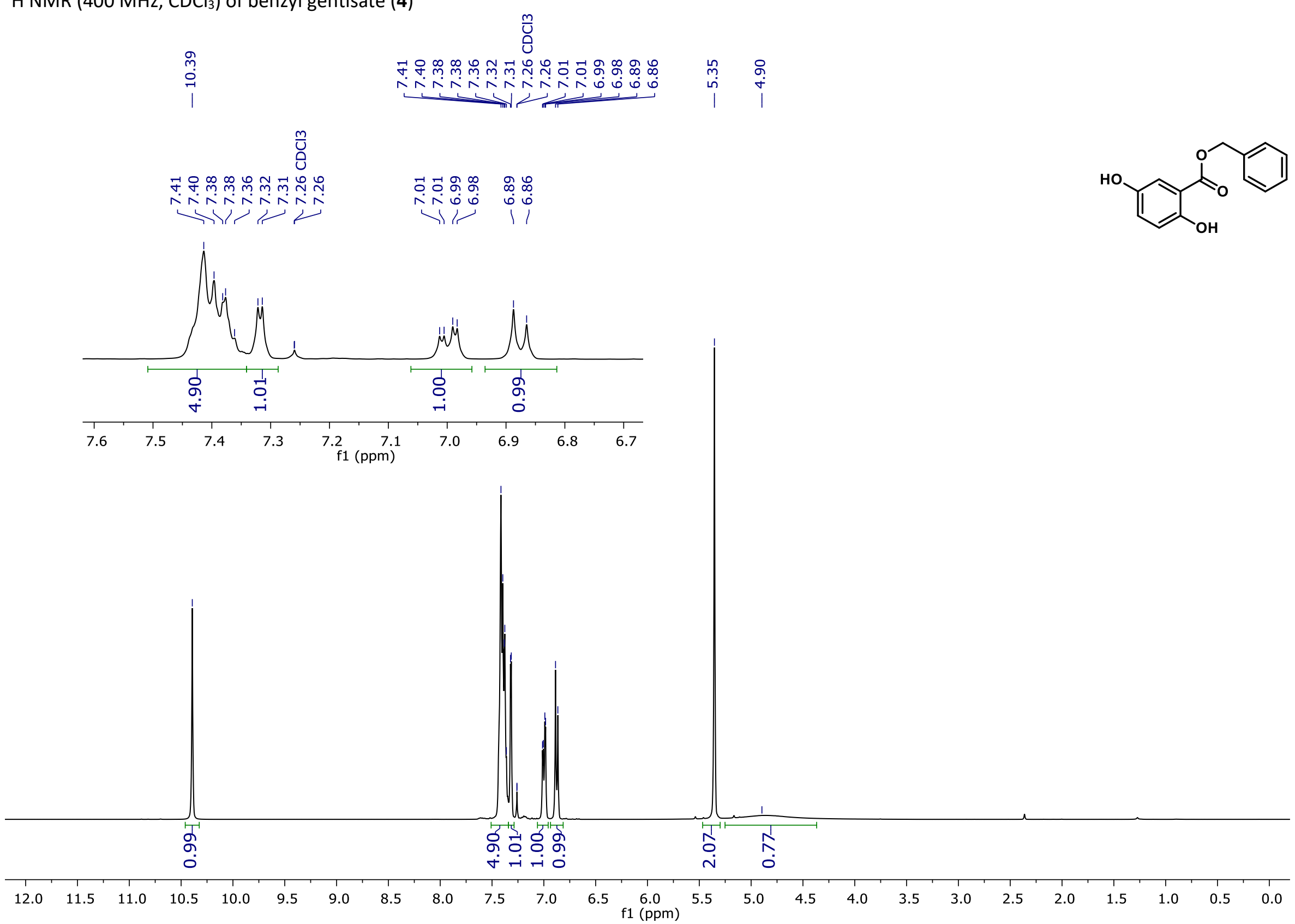


${ }^{13} \mathrm{C}$ NMR (101 MHz, $\mathrm{CDCl}_{3}$ ) of of benzyl gentisate (4)

$$
\text { م) }
$$

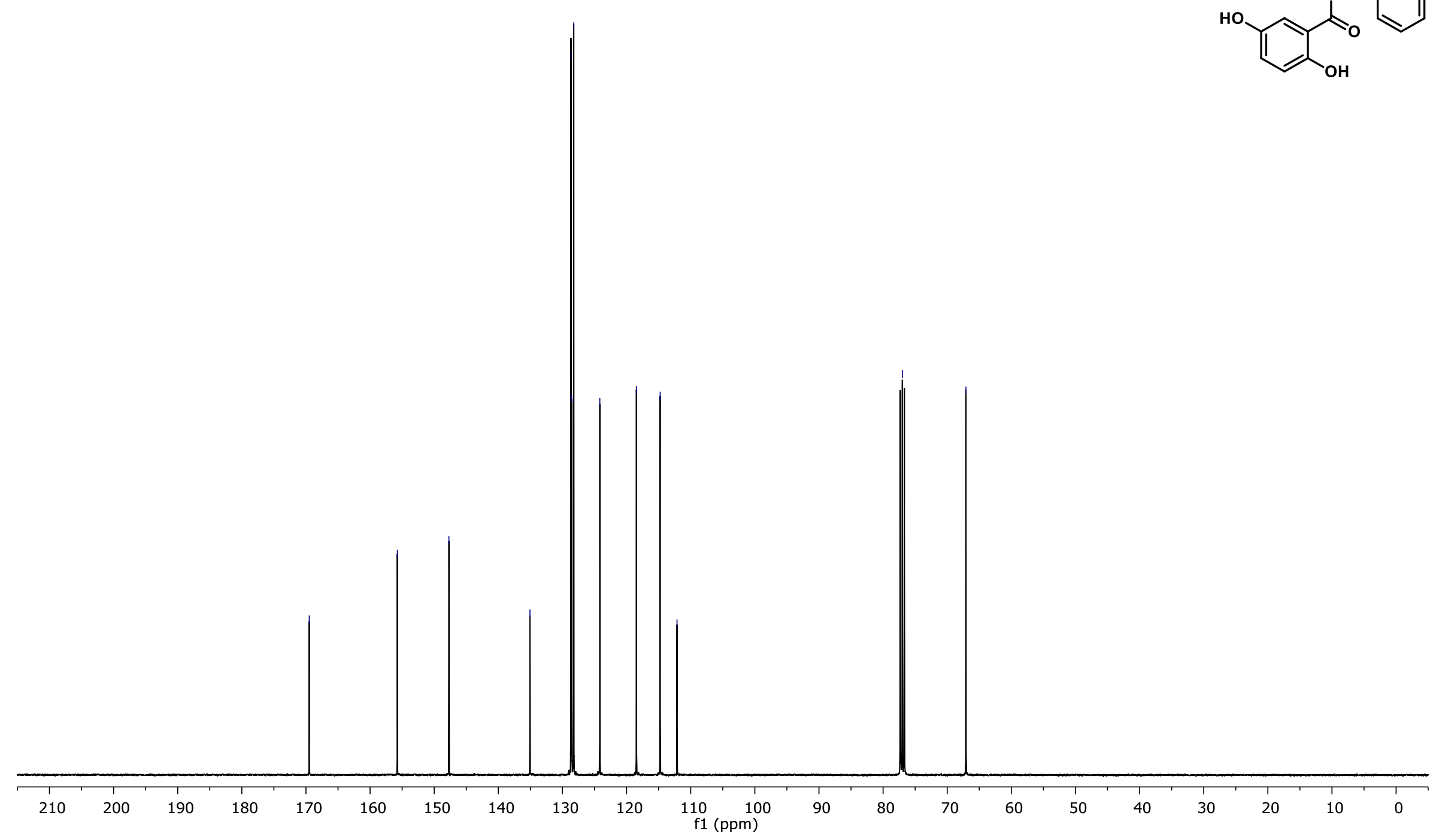


ATR-FTIR and UV of benzyl gentisate (4)
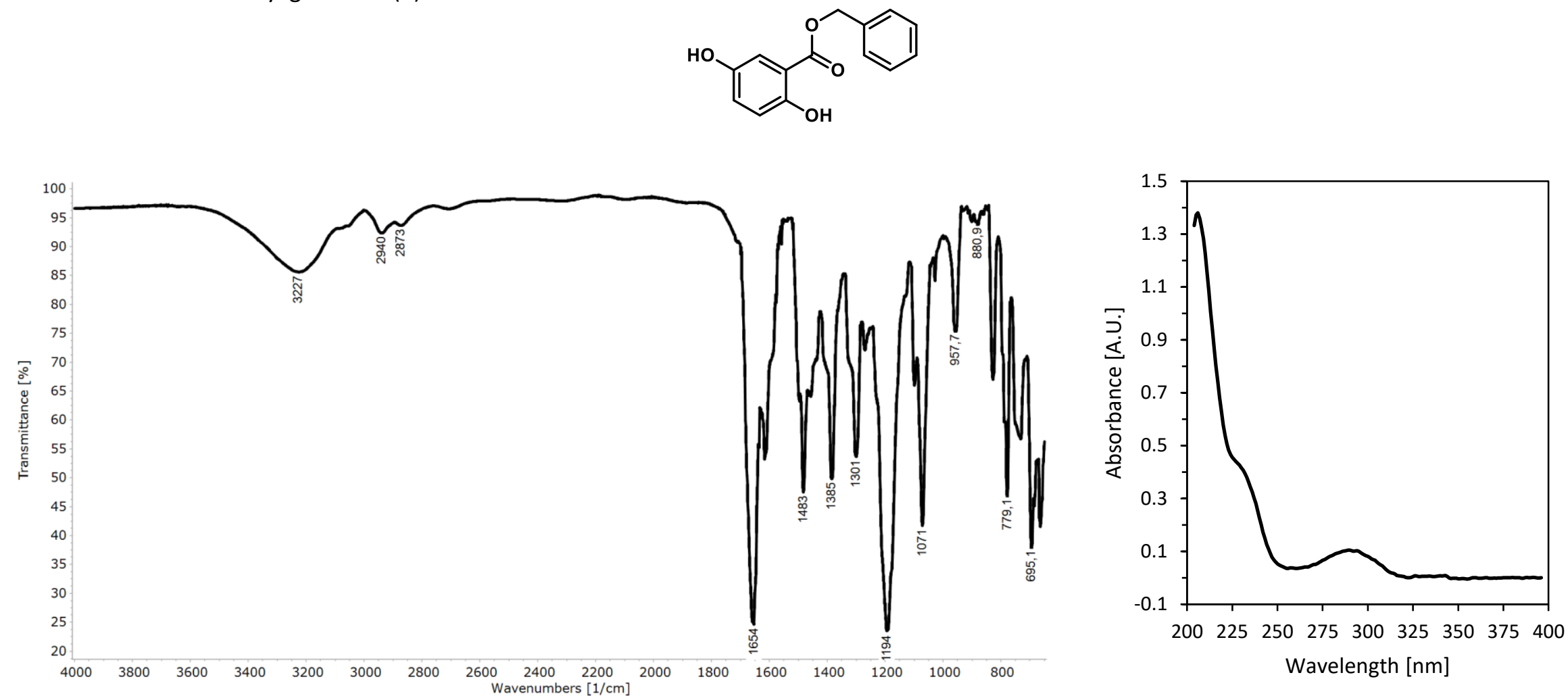
Trichocarpine (5)

${ }^{1} \mathrm{H}$ NMR (400 MHz, MeOD) of trichocarpine (5)

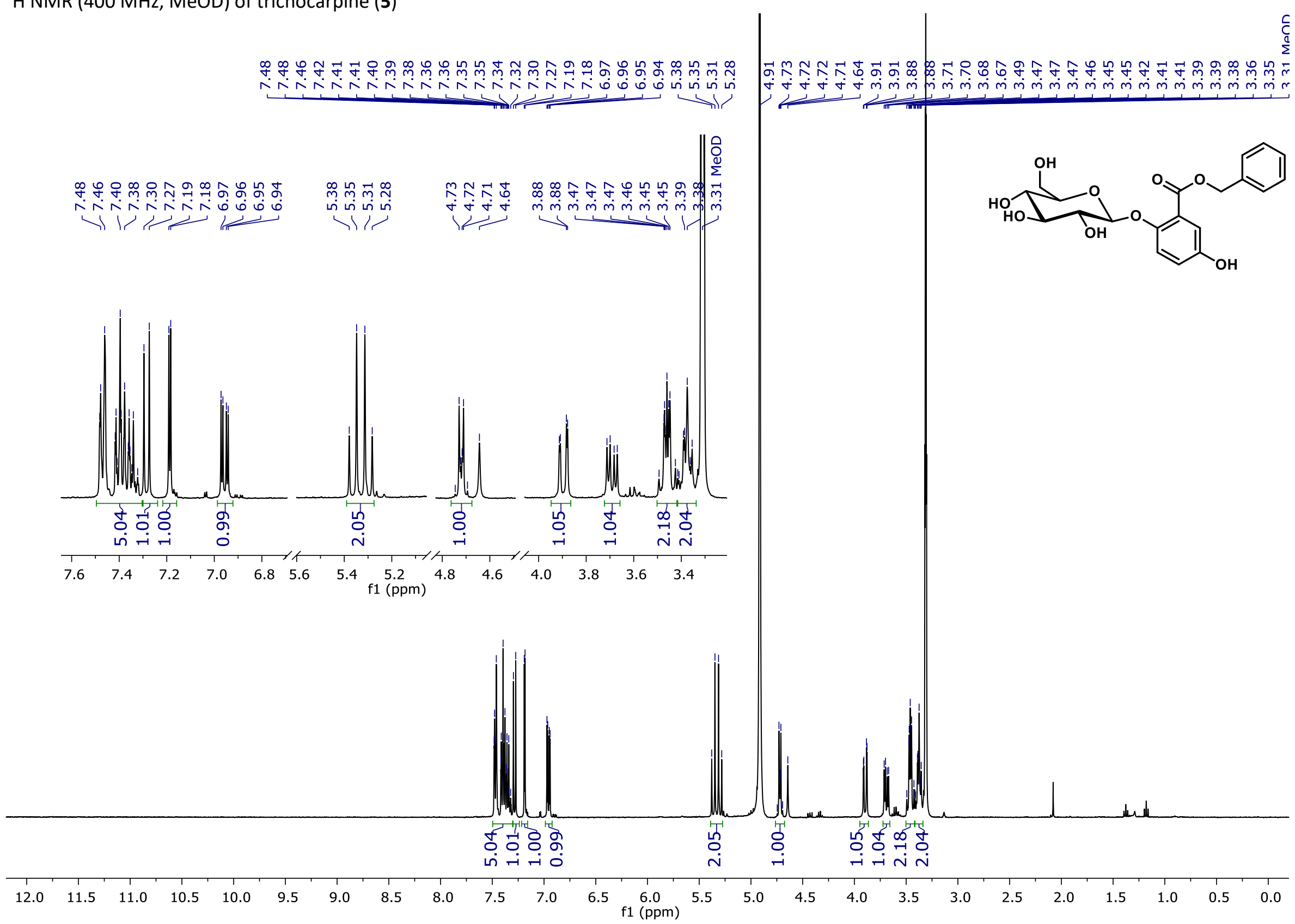


${ }^{13} \mathrm{C}$ NMR (101 MHz, MeOD) of trichocarpine (5)

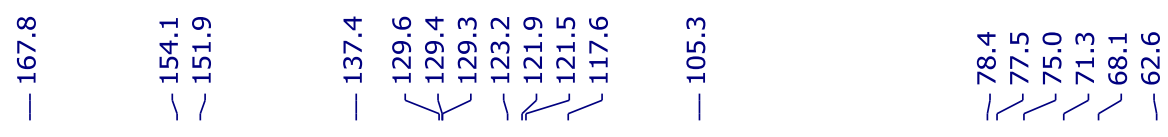

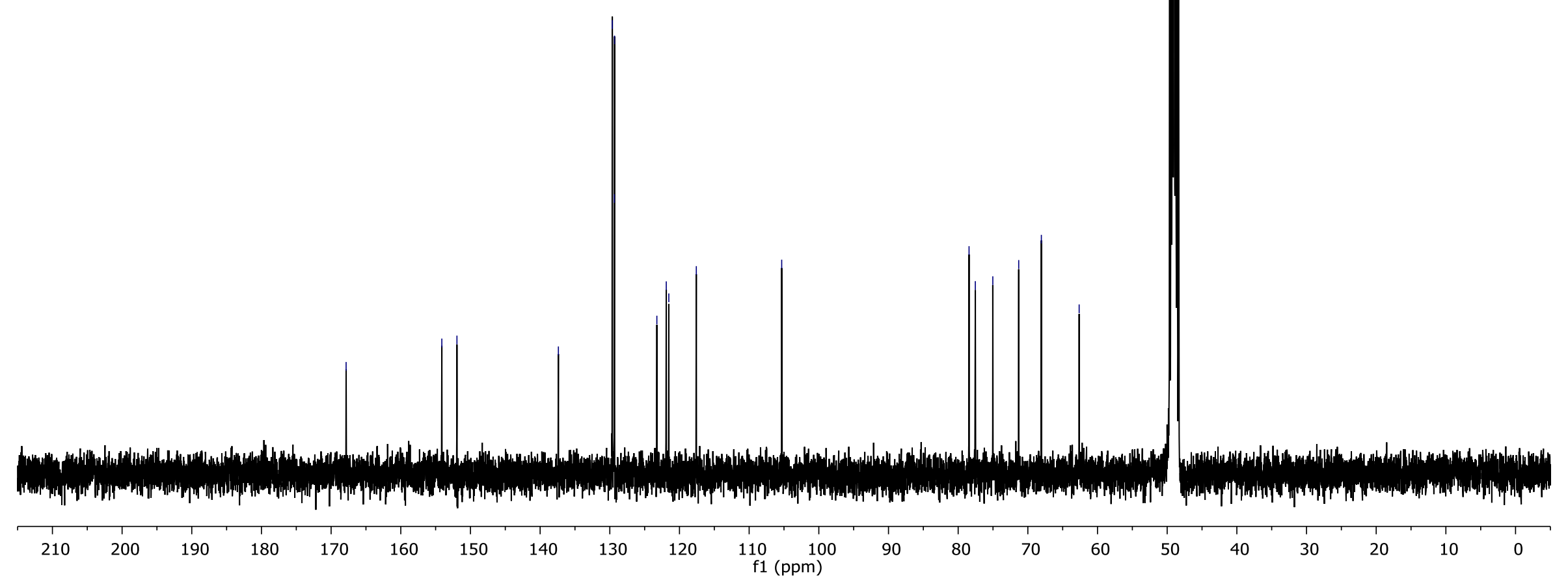




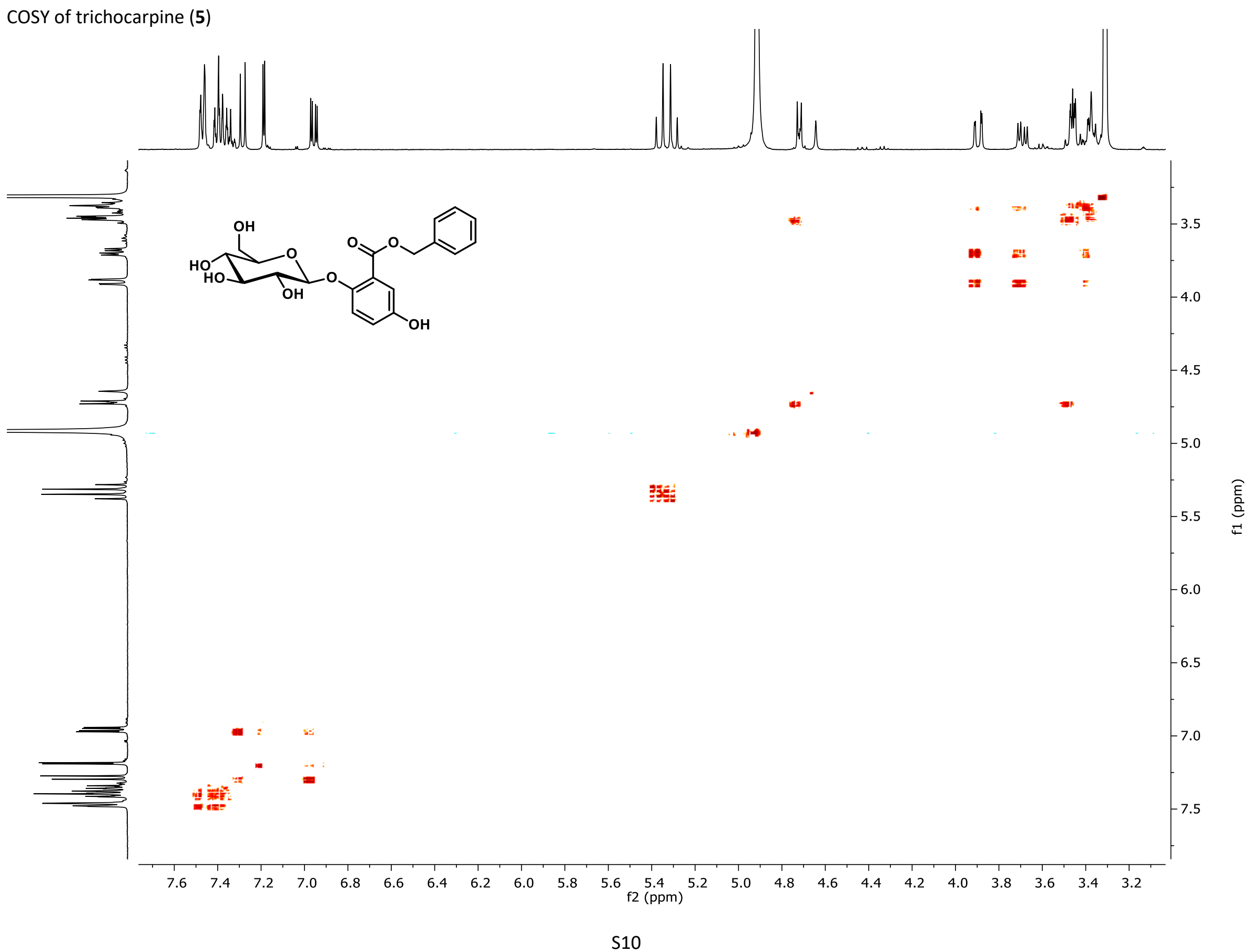


HSQC of trichocarpine (5)

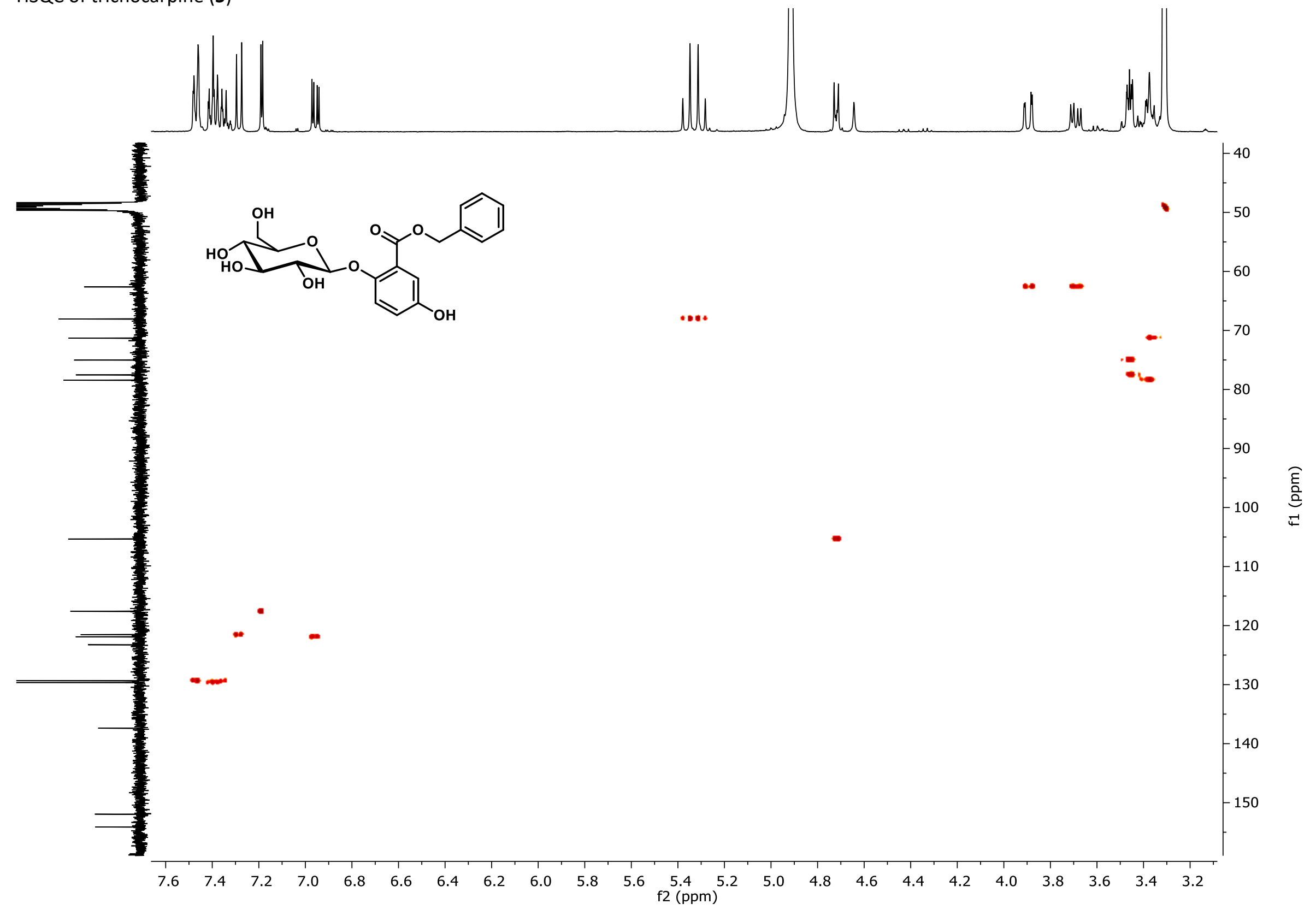


ATR-FTIR and UV of trichocarpine (5)
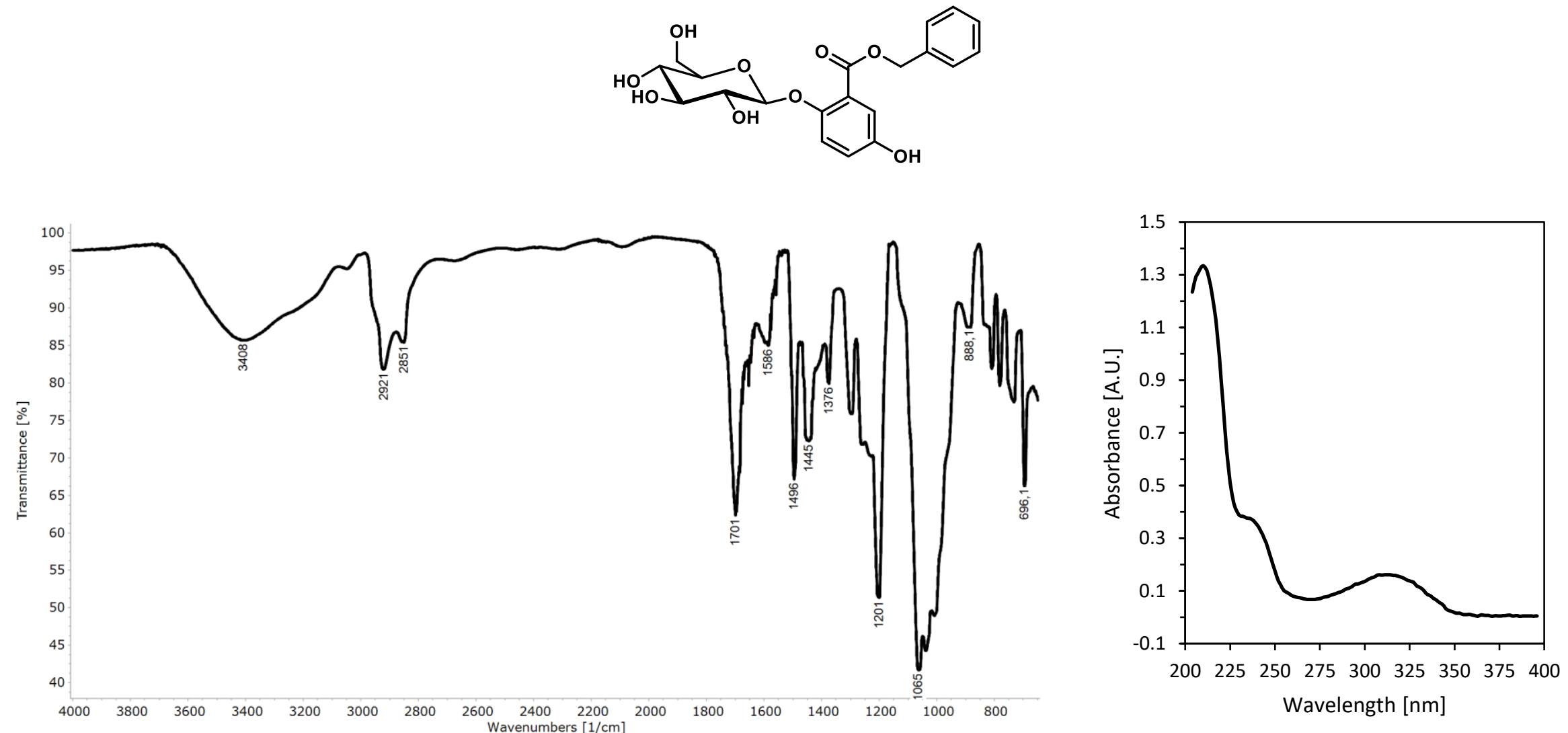


\section{2-0-Acety/trichocarpine (6)}

${ }^{1} \mathrm{H}$ NMR (400 MHz, MeOD) of 2-O-acetyltrichocarpine (6)

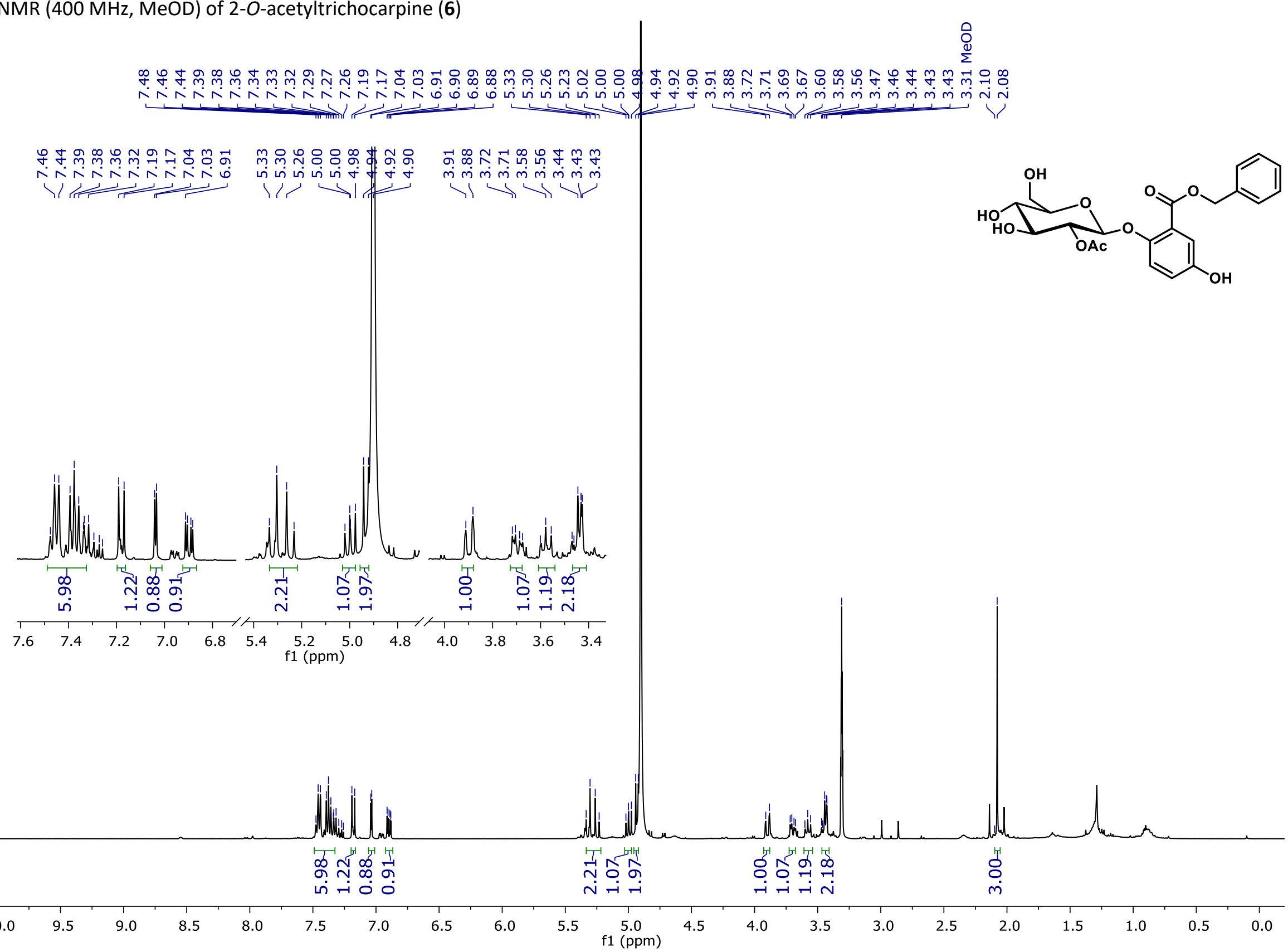


${ }^{13} \mathrm{C}$ NMR (101 MHz, MeOD) of 2-O-acetyltrichocarpine (6)

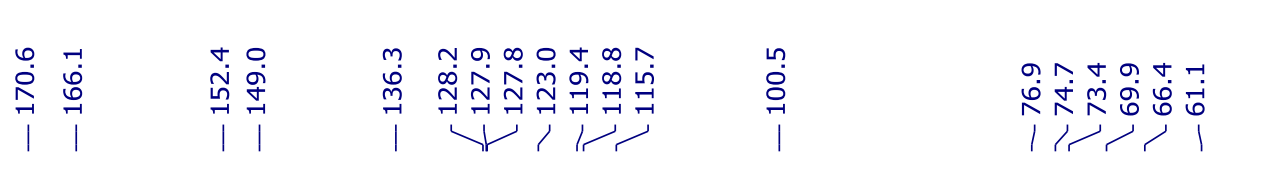
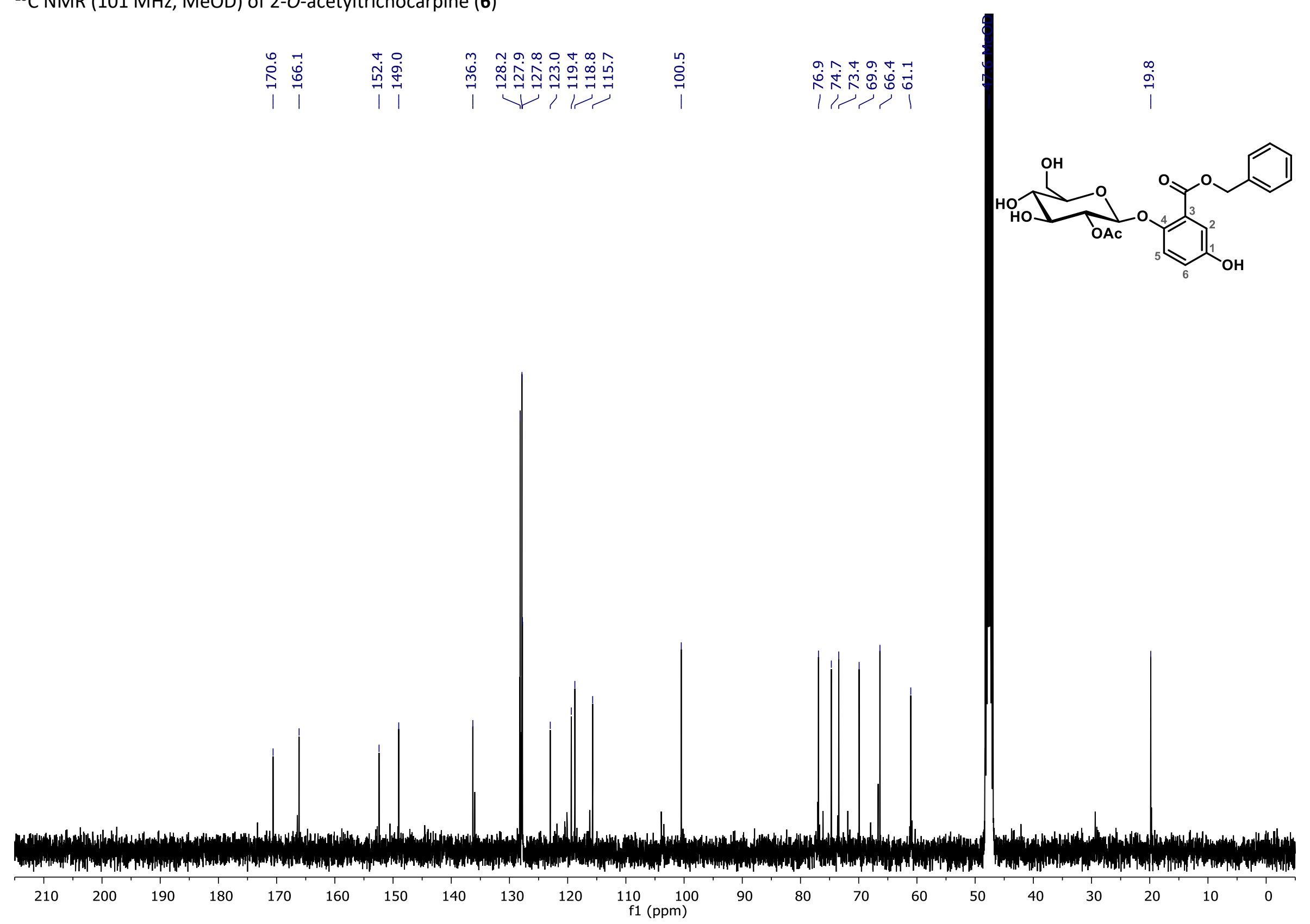


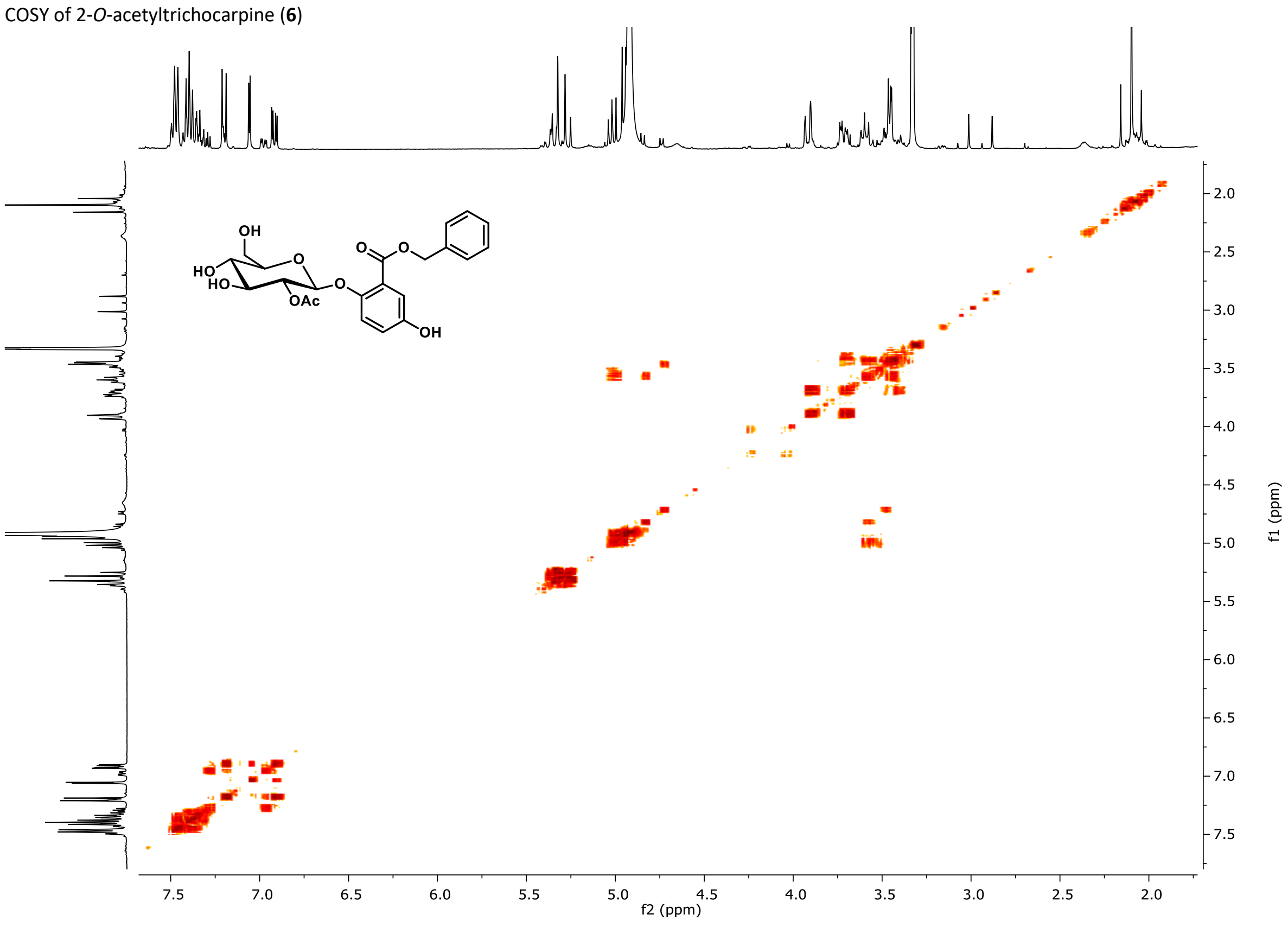


HSQC of 2-O-acetyltrichocarpine (6)

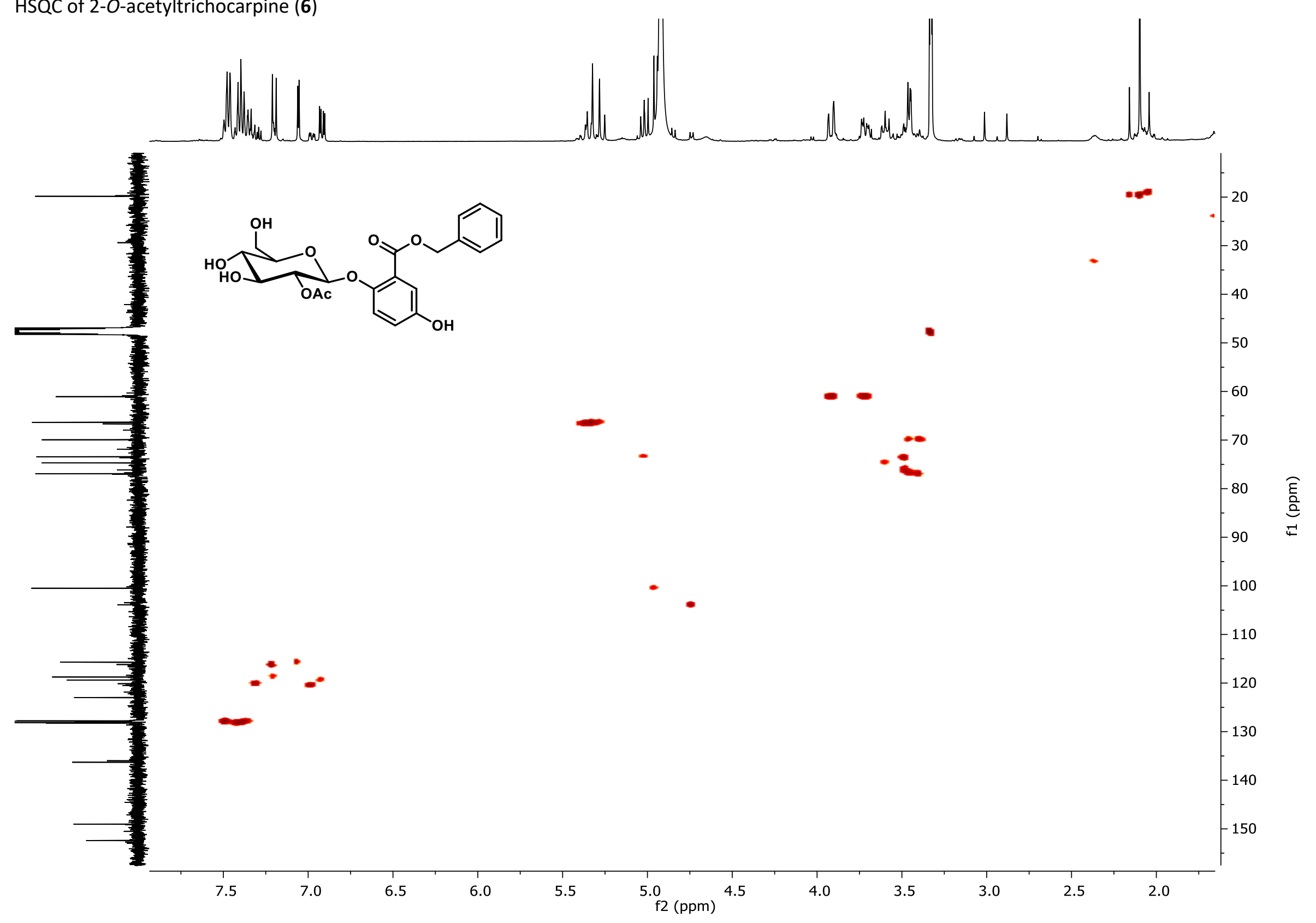


ATR-FTIR and UV of 2-O-acetyltrichocarpine (6)
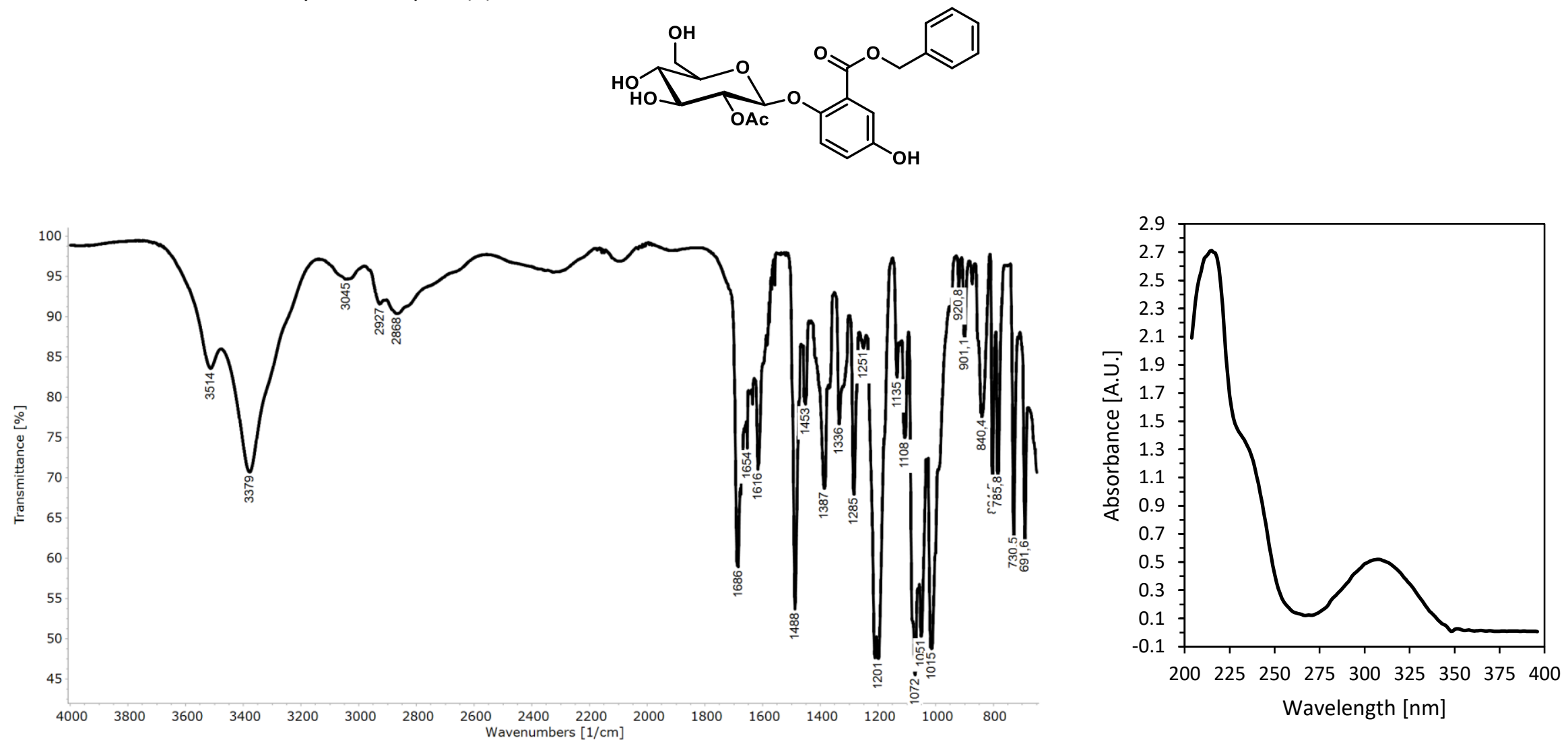


\section{Isotrichocarpine (7)}

${ }^{1} \mathrm{H}$ NMR (400 MHz, DMSO- $d_{6}$ ) of isotrichocarpine (7)

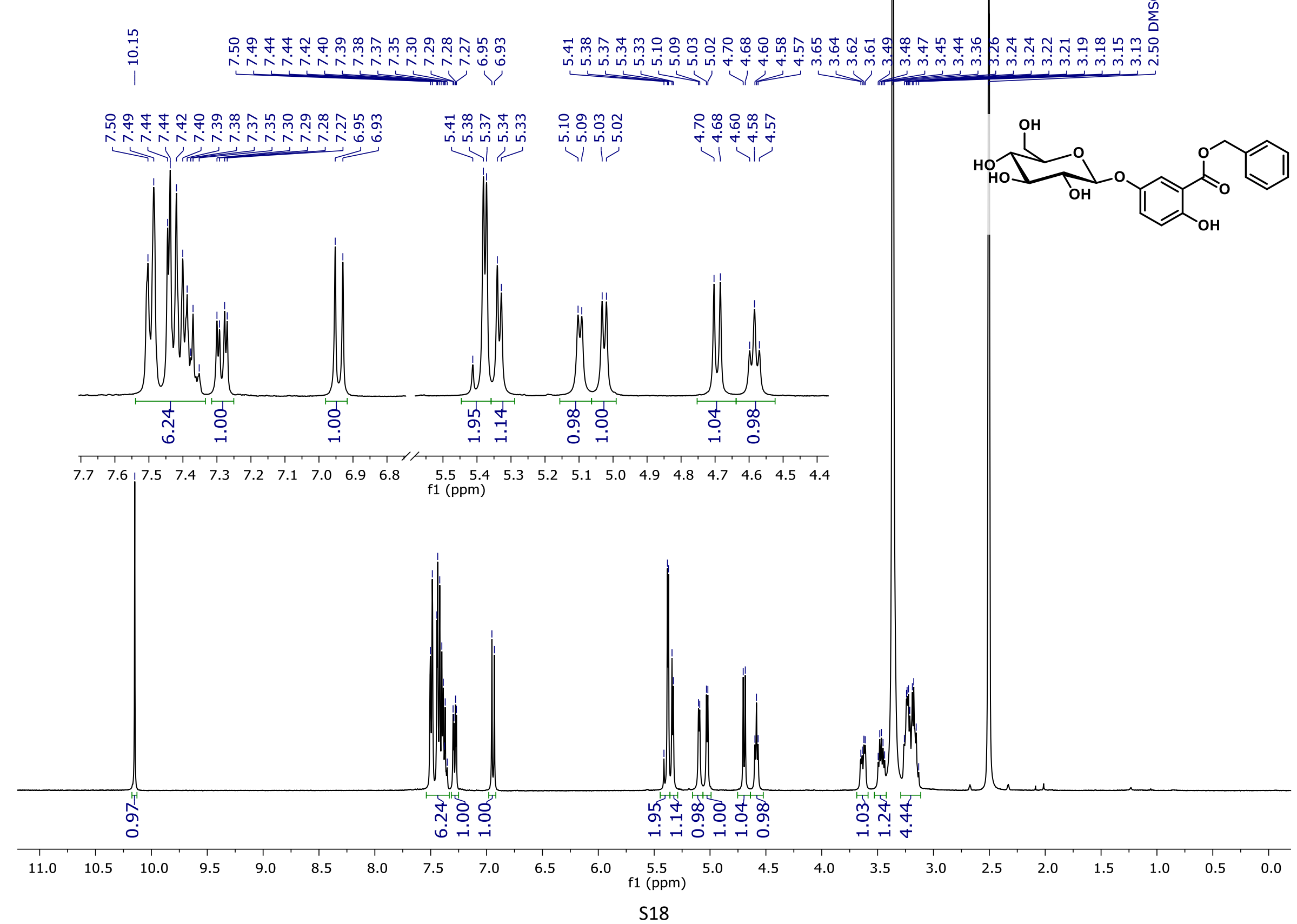


${ }^{13} \mathrm{C}$ NMR (101 MHz, DMSO- $d_{6}$ ) of isotrichocarpine (7)

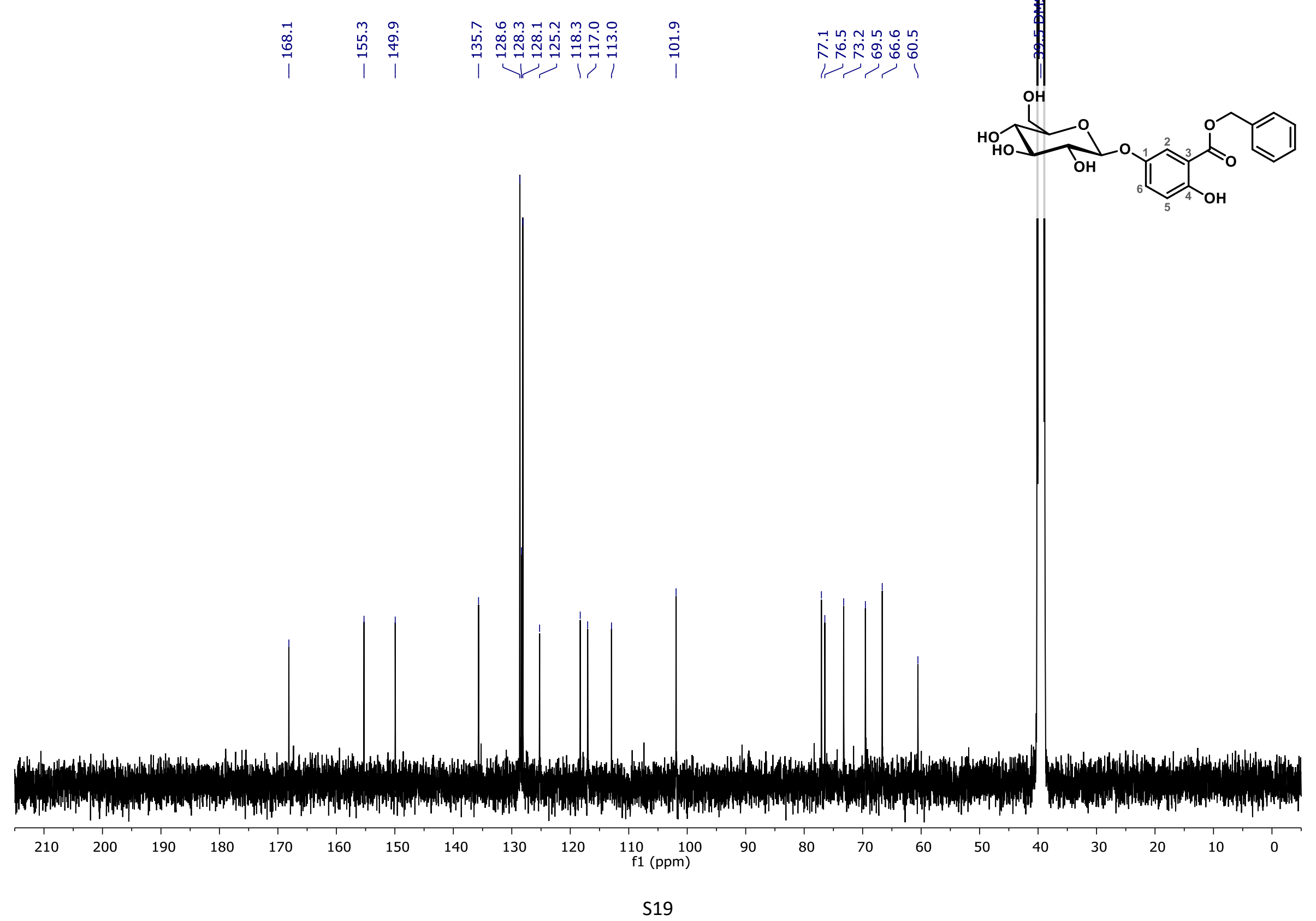




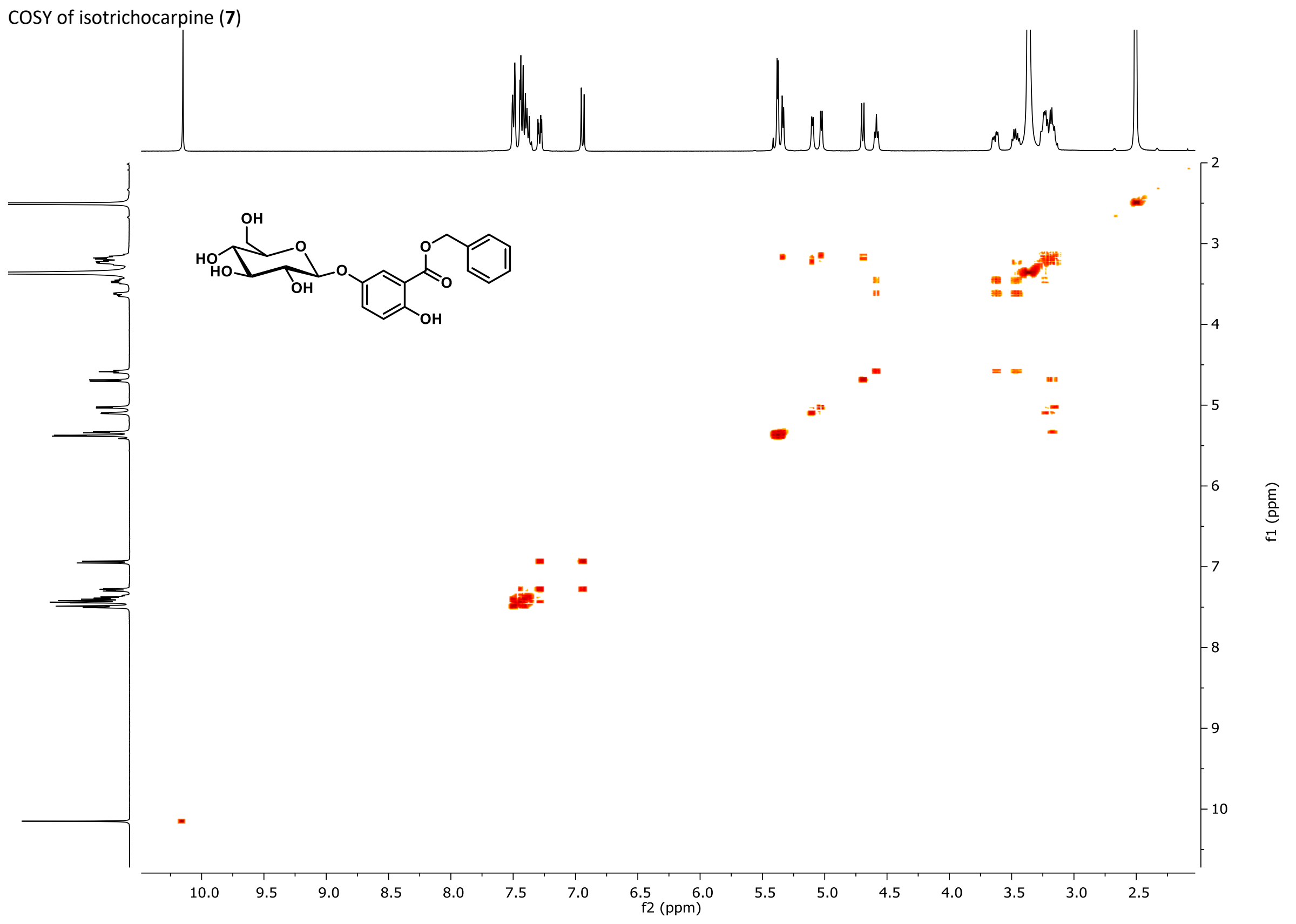


HSQC of isotrichocarpine (7)

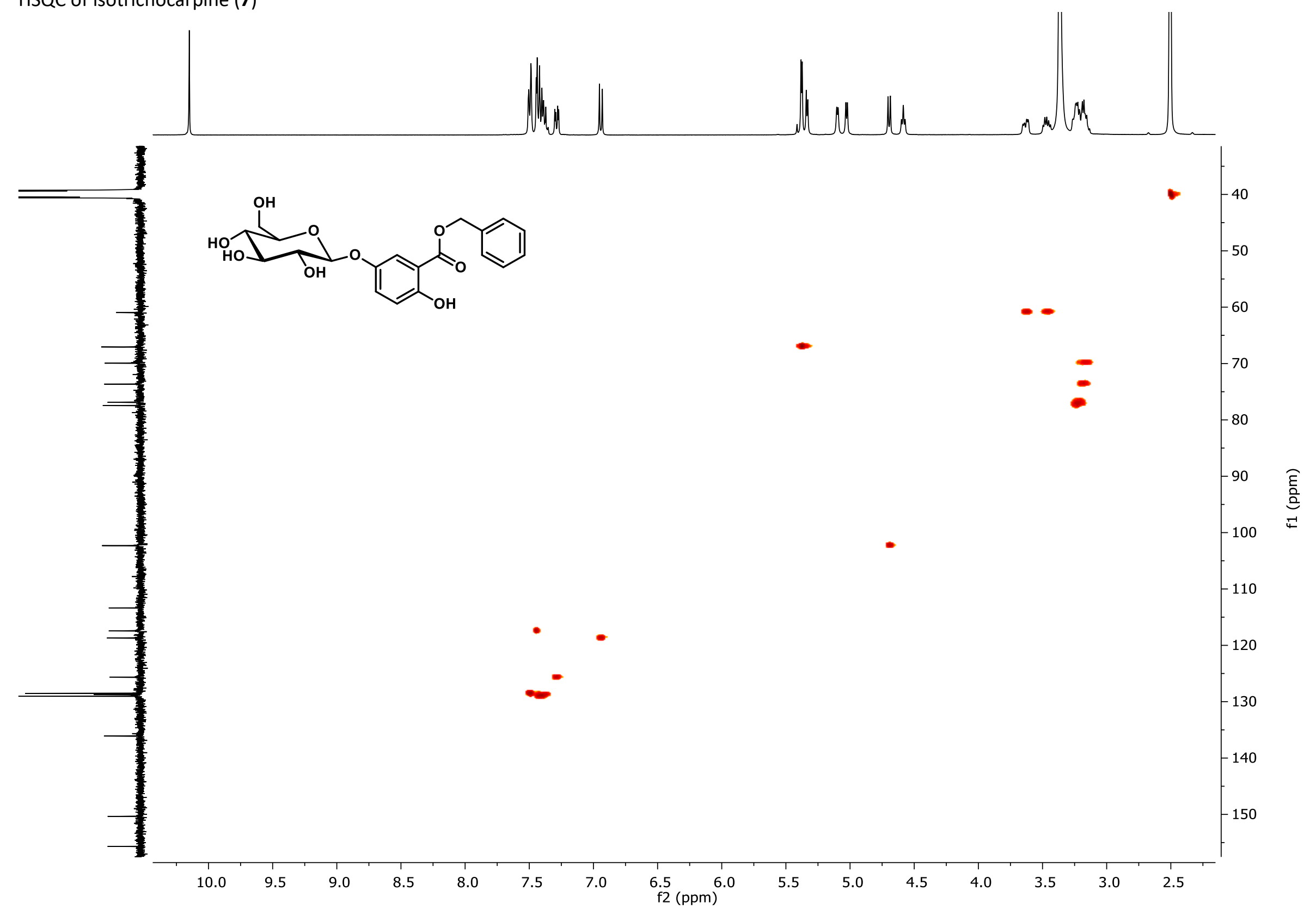


ATR-FTIR and UV of isotrichocarpine (7)
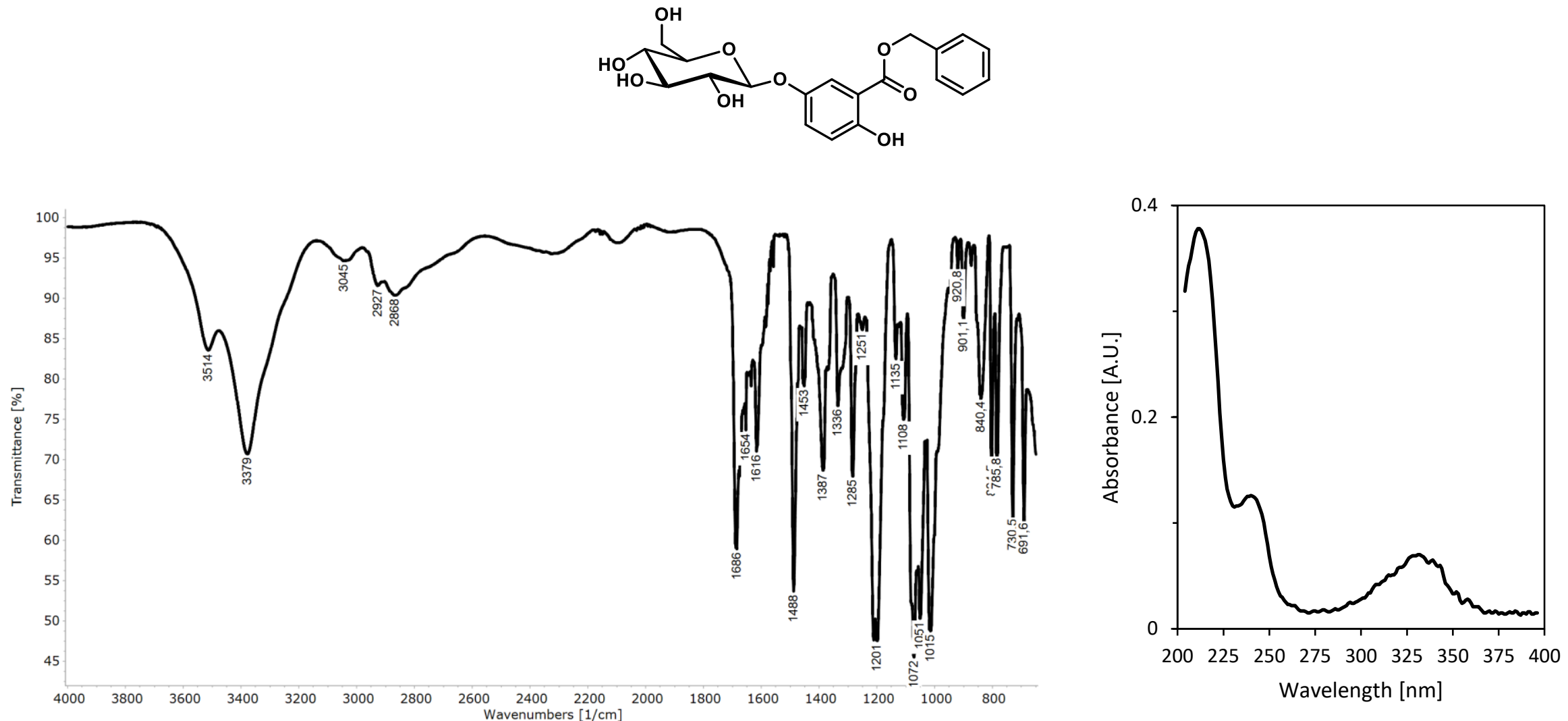
Trichoside (8)

${ }^{1} \mathrm{H}$ NMR (400 MHz, MeOD) of trichoside (8)

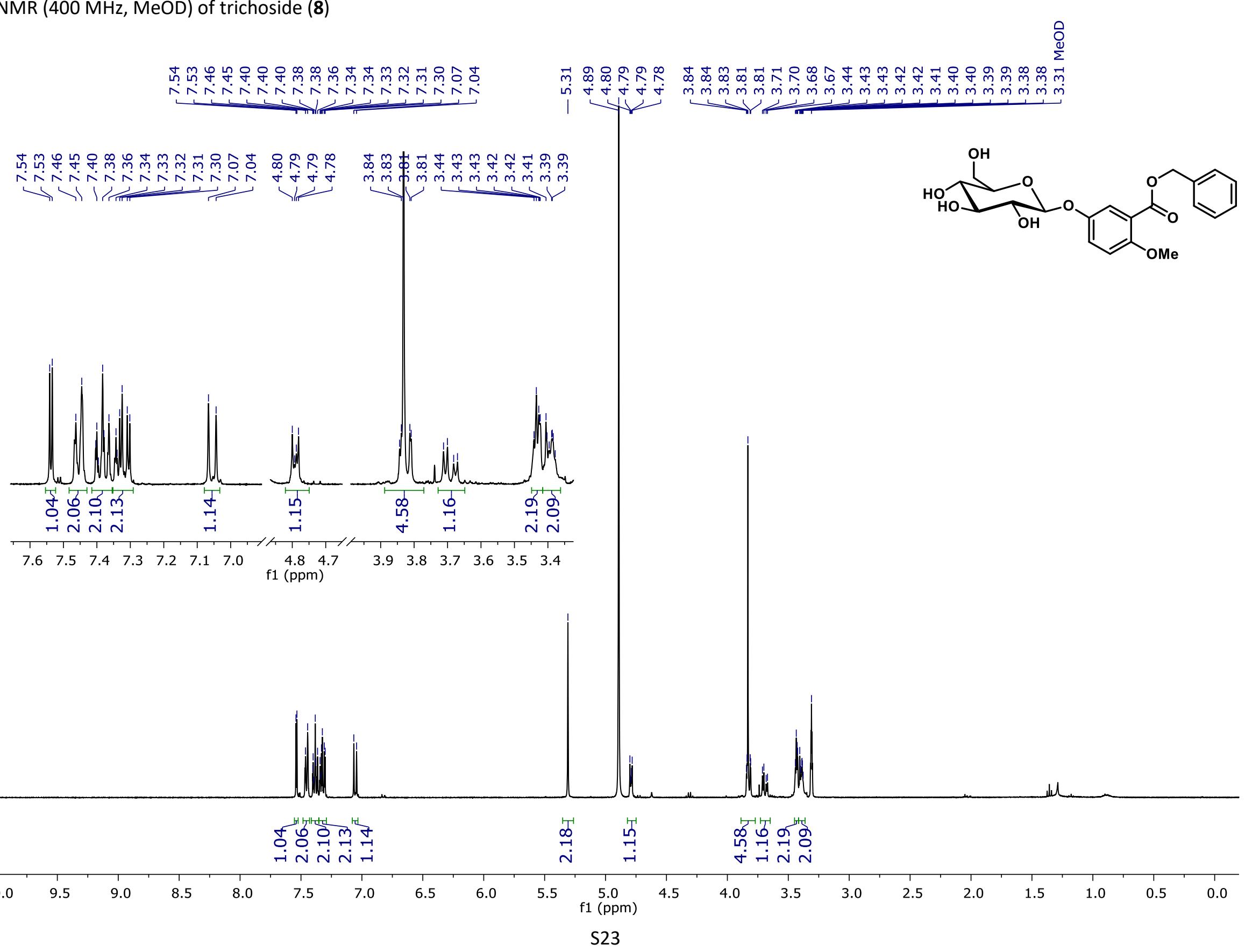


${ }^{13} \mathrm{C}$ NMR (101 MHz, MeOD) of trichoside (8)
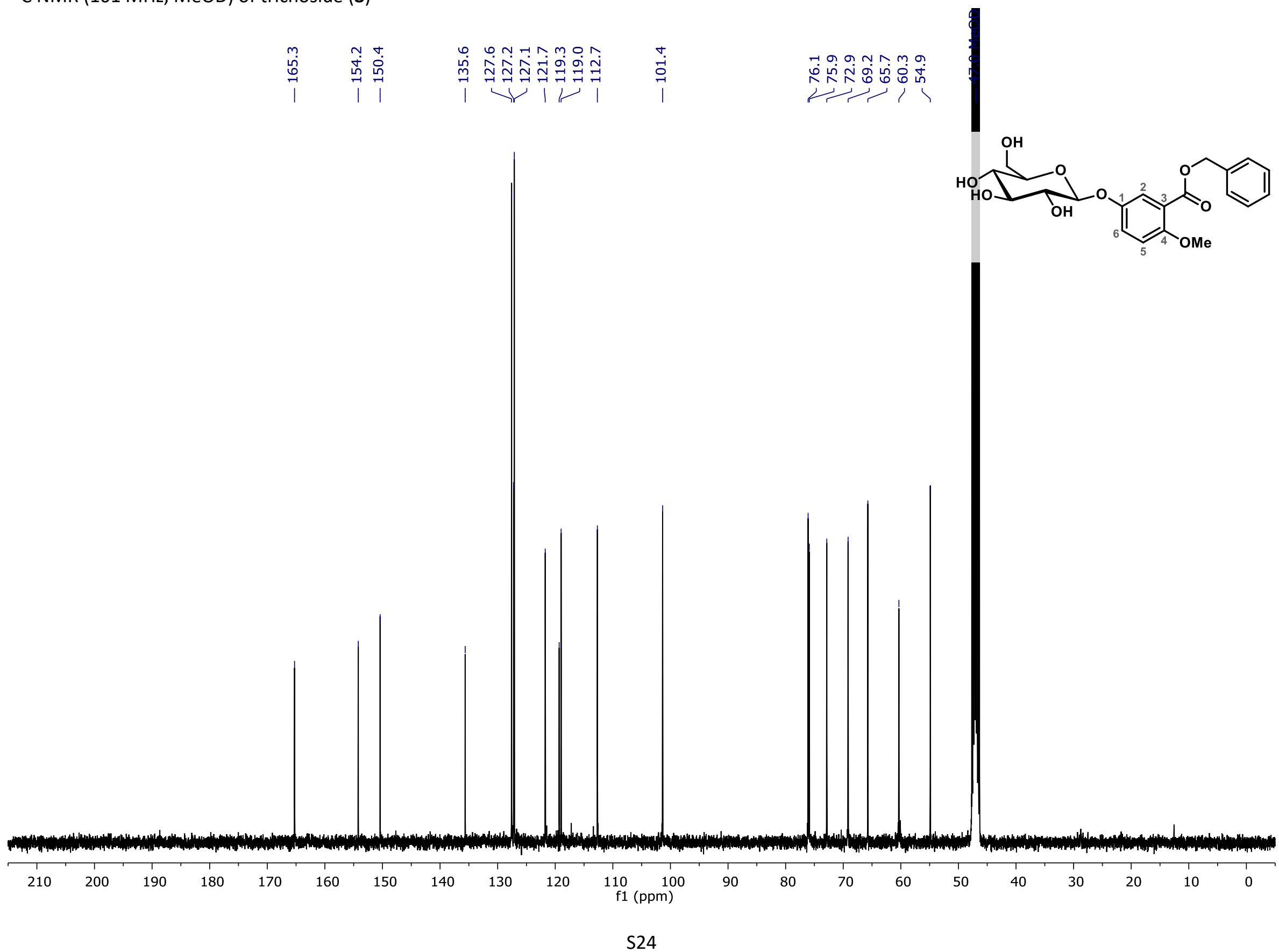
COSY of trichoside (8)

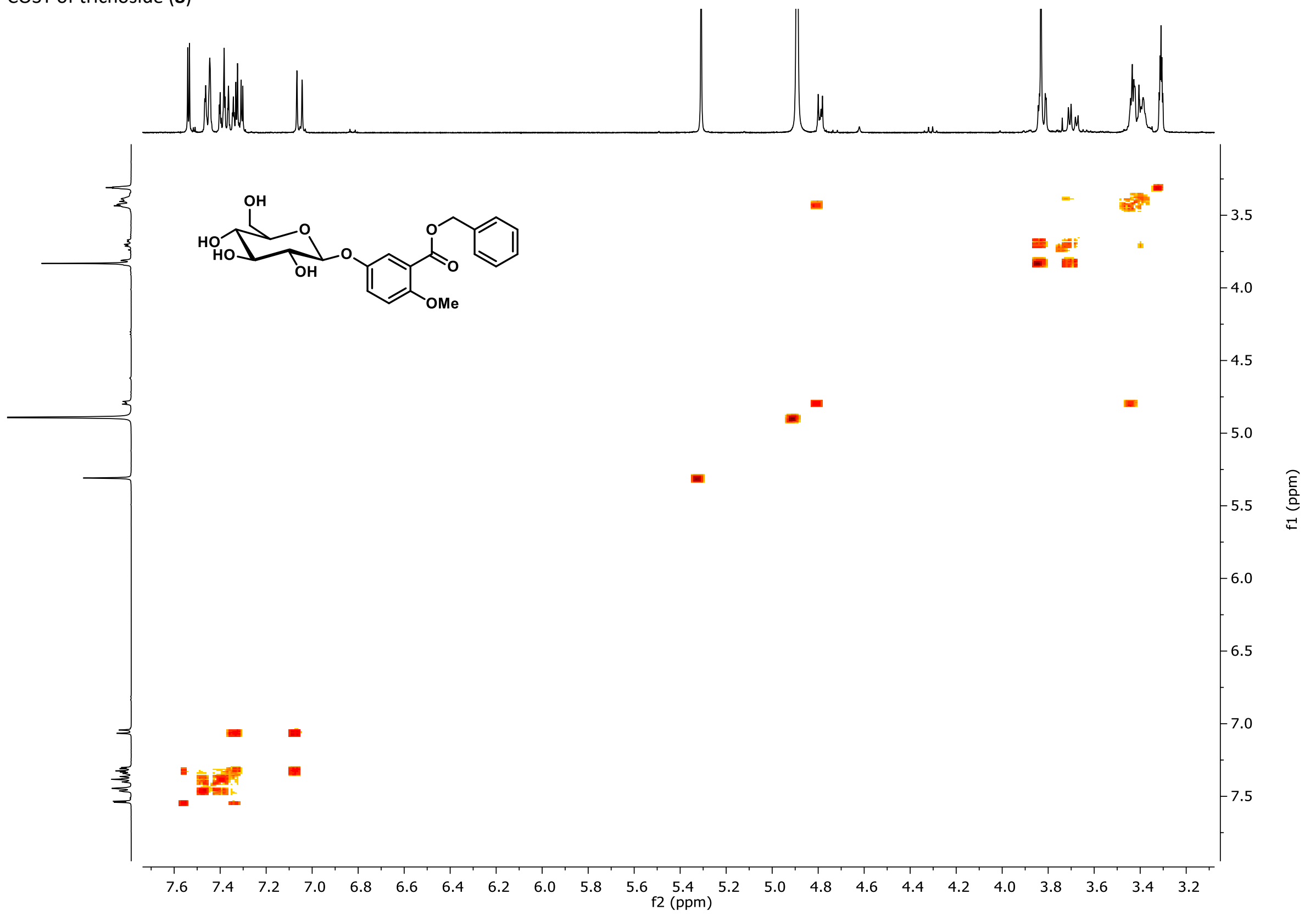


HSQC of trichoside (8)

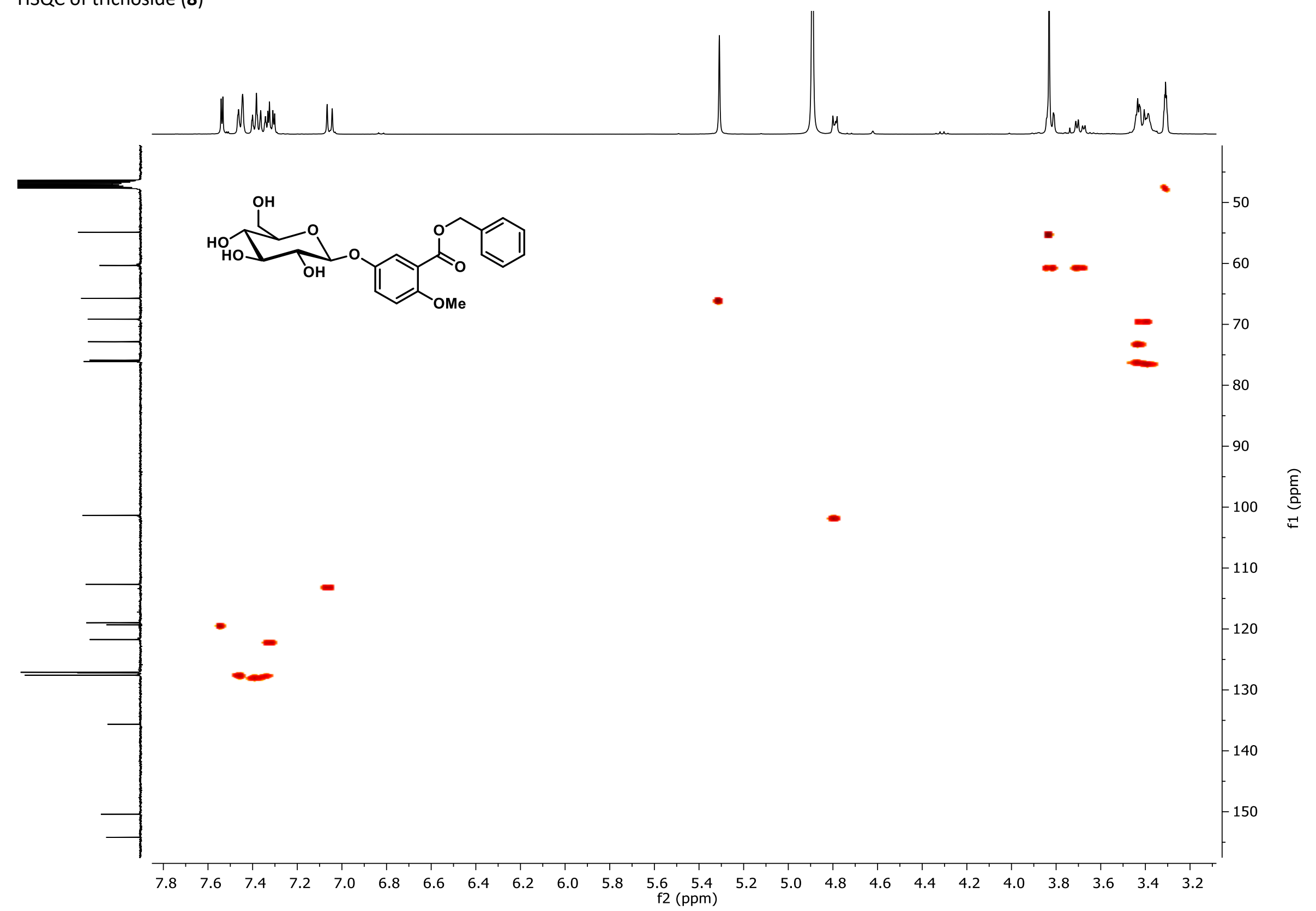



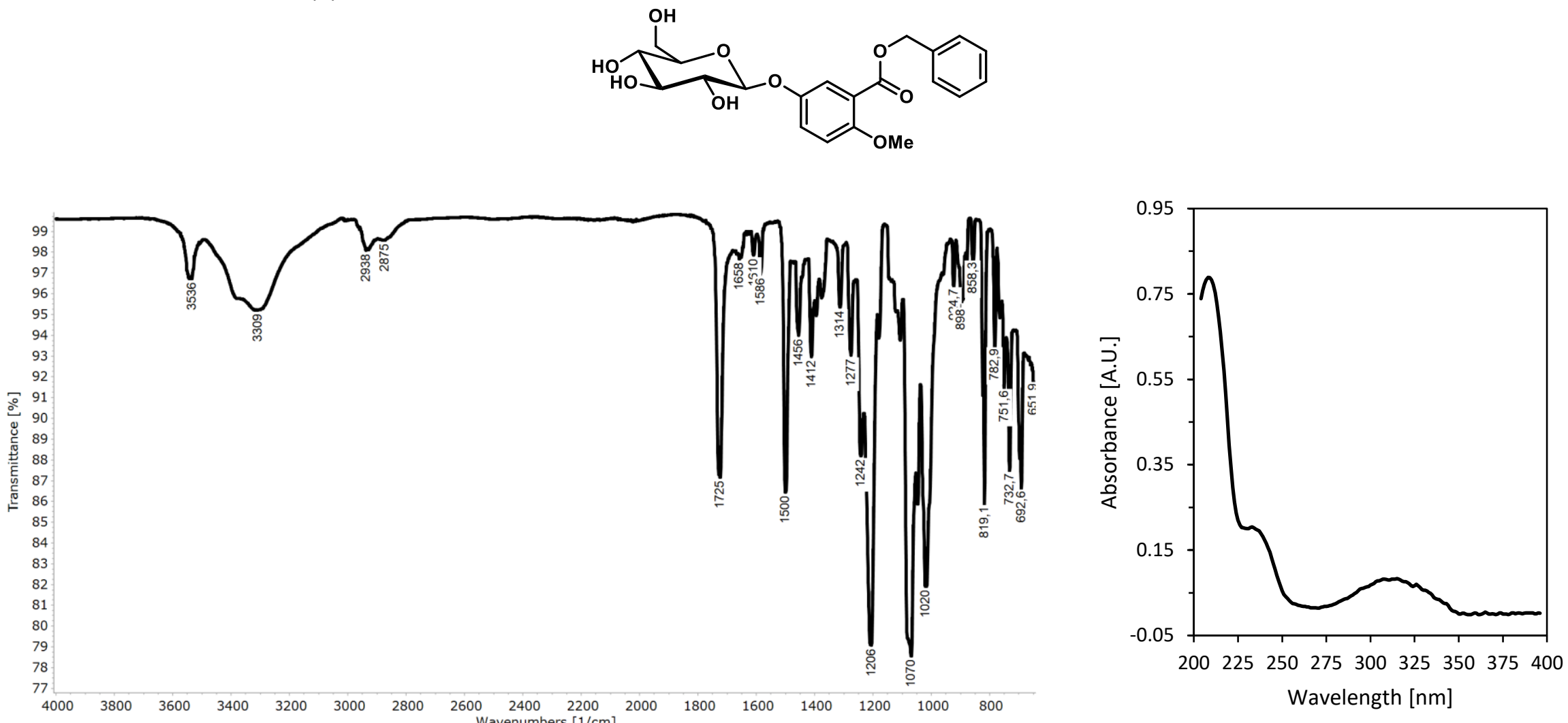


\section{Deoxytrichocarpine (9)}

${ }^{1} \mathrm{H}$ NMR (400 MHz, MeOD) of deoxytrichocarpine (9)

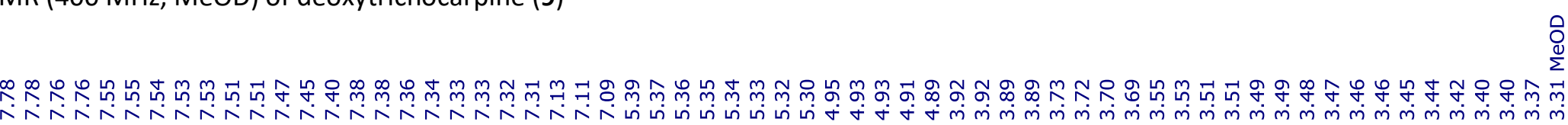
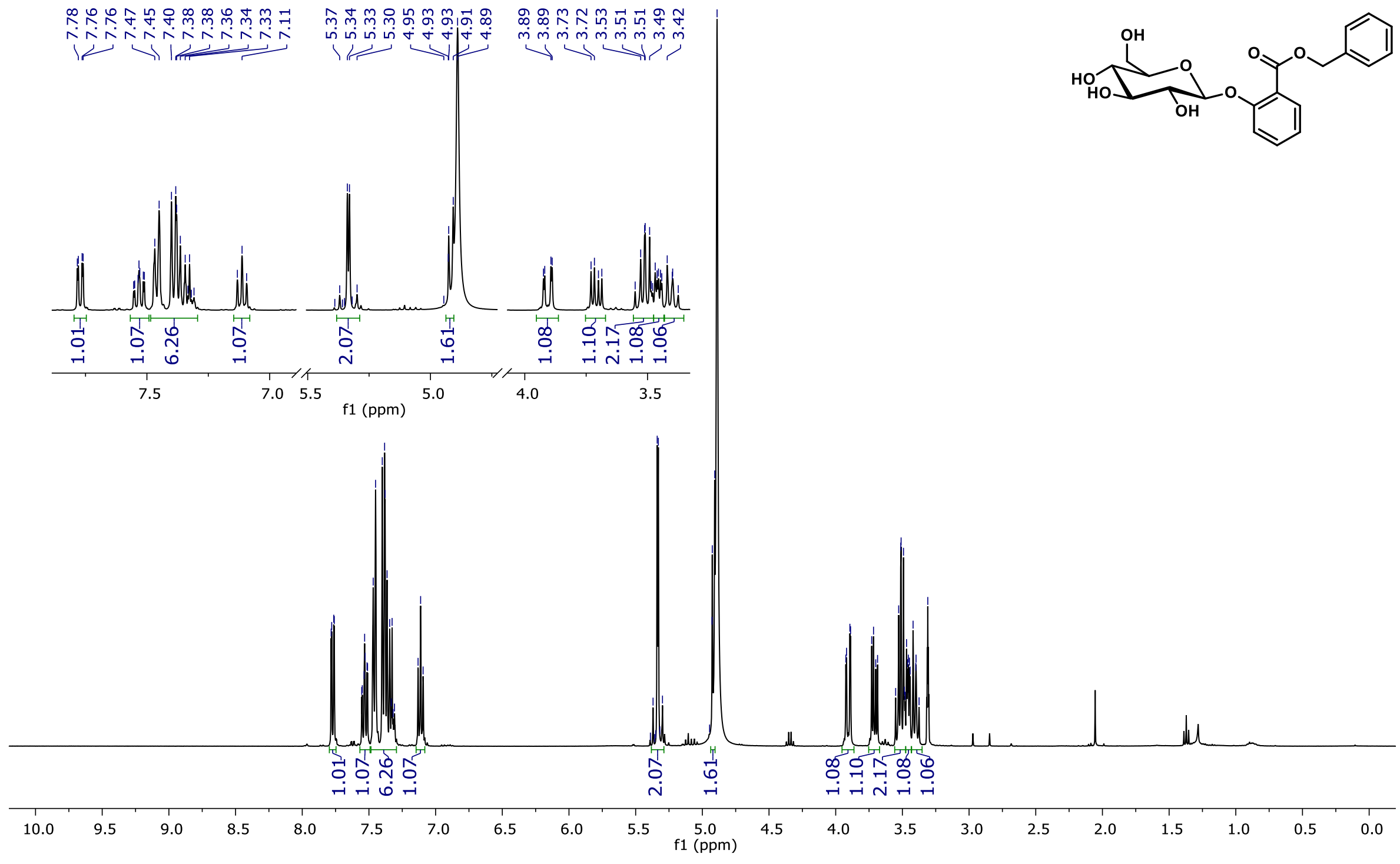
${ }^{13} \mathrm{C}$ NMR (101 MHz, MeOD) of deoxytrichocarpine (9)

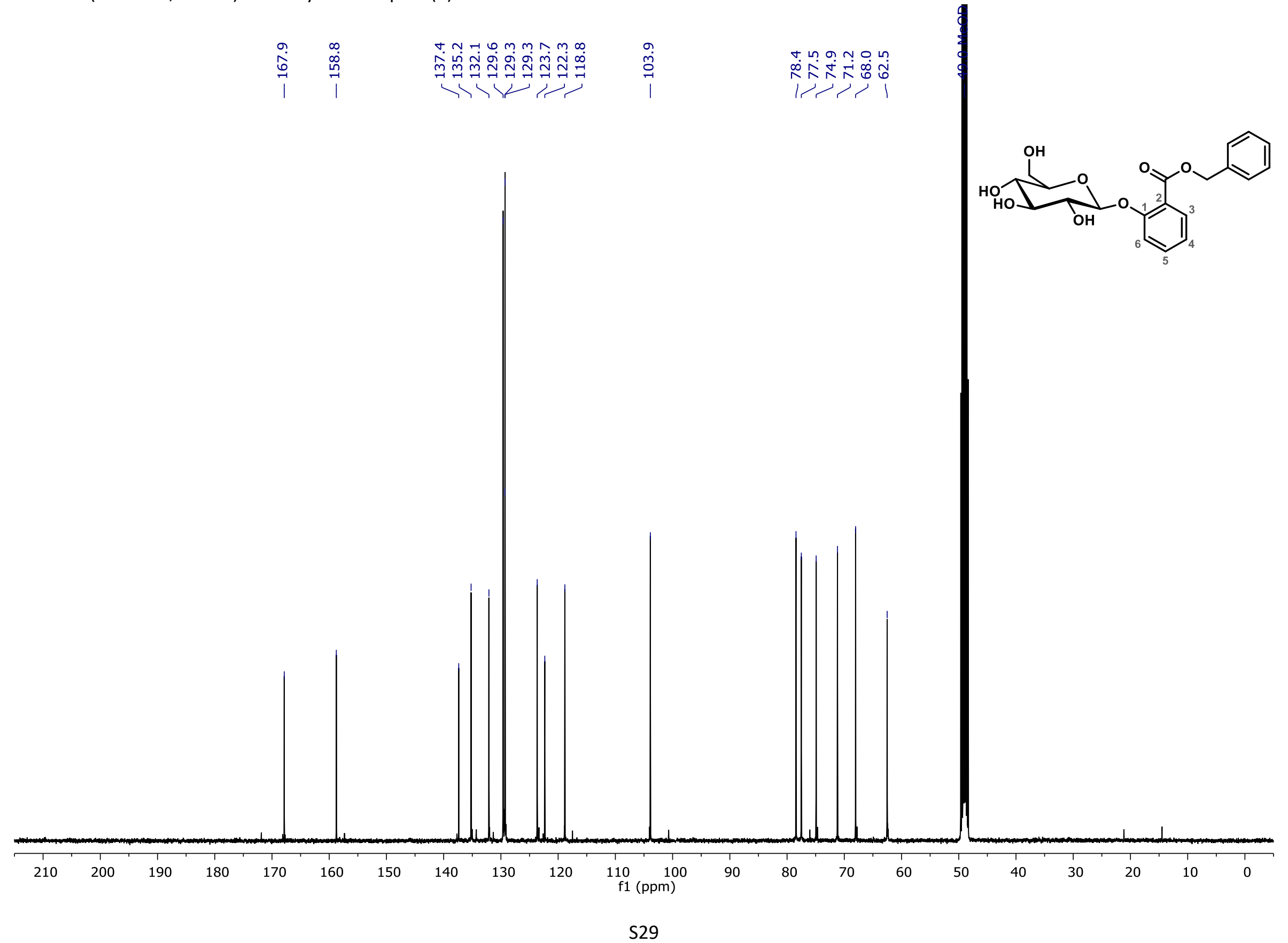




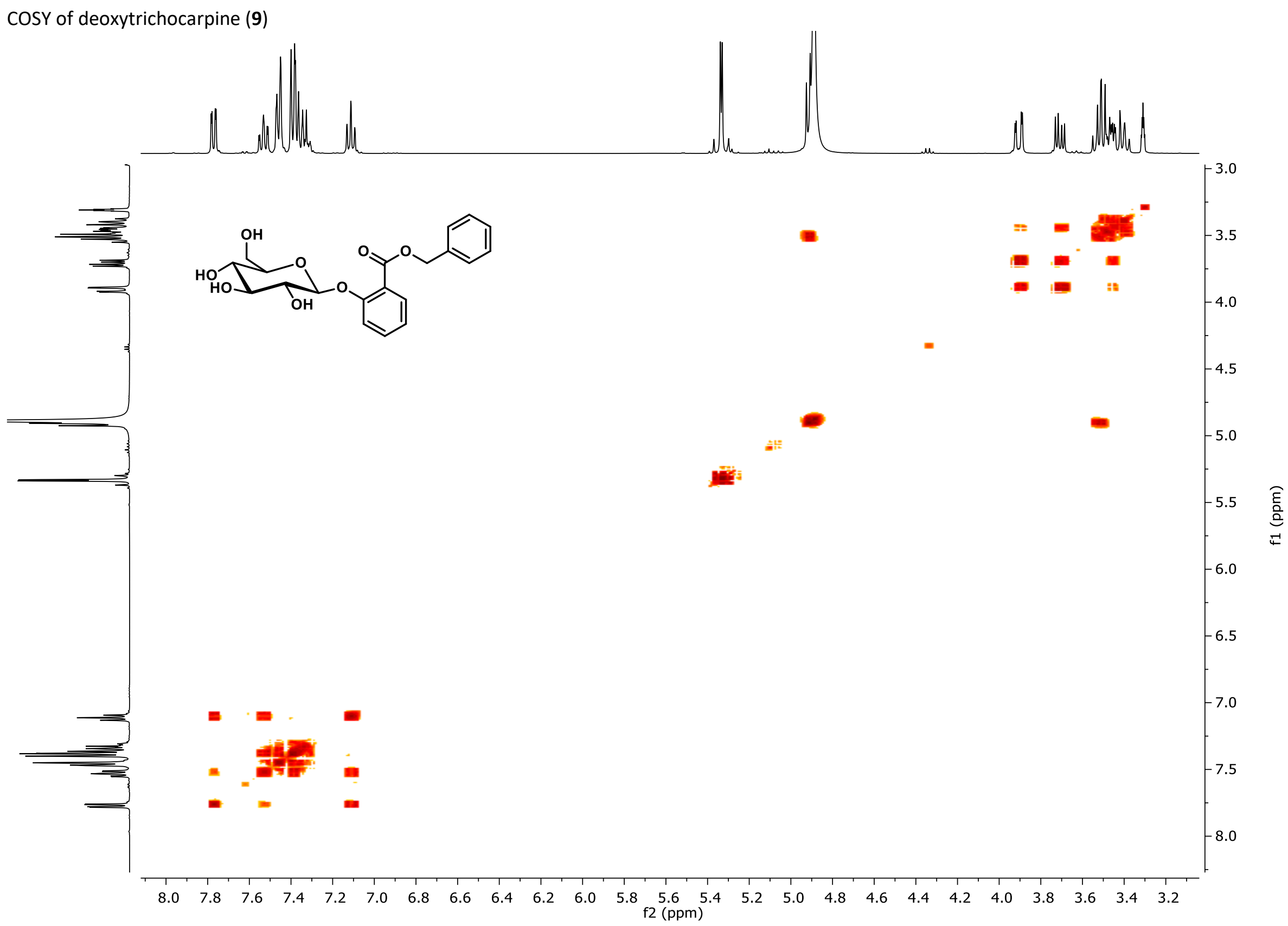




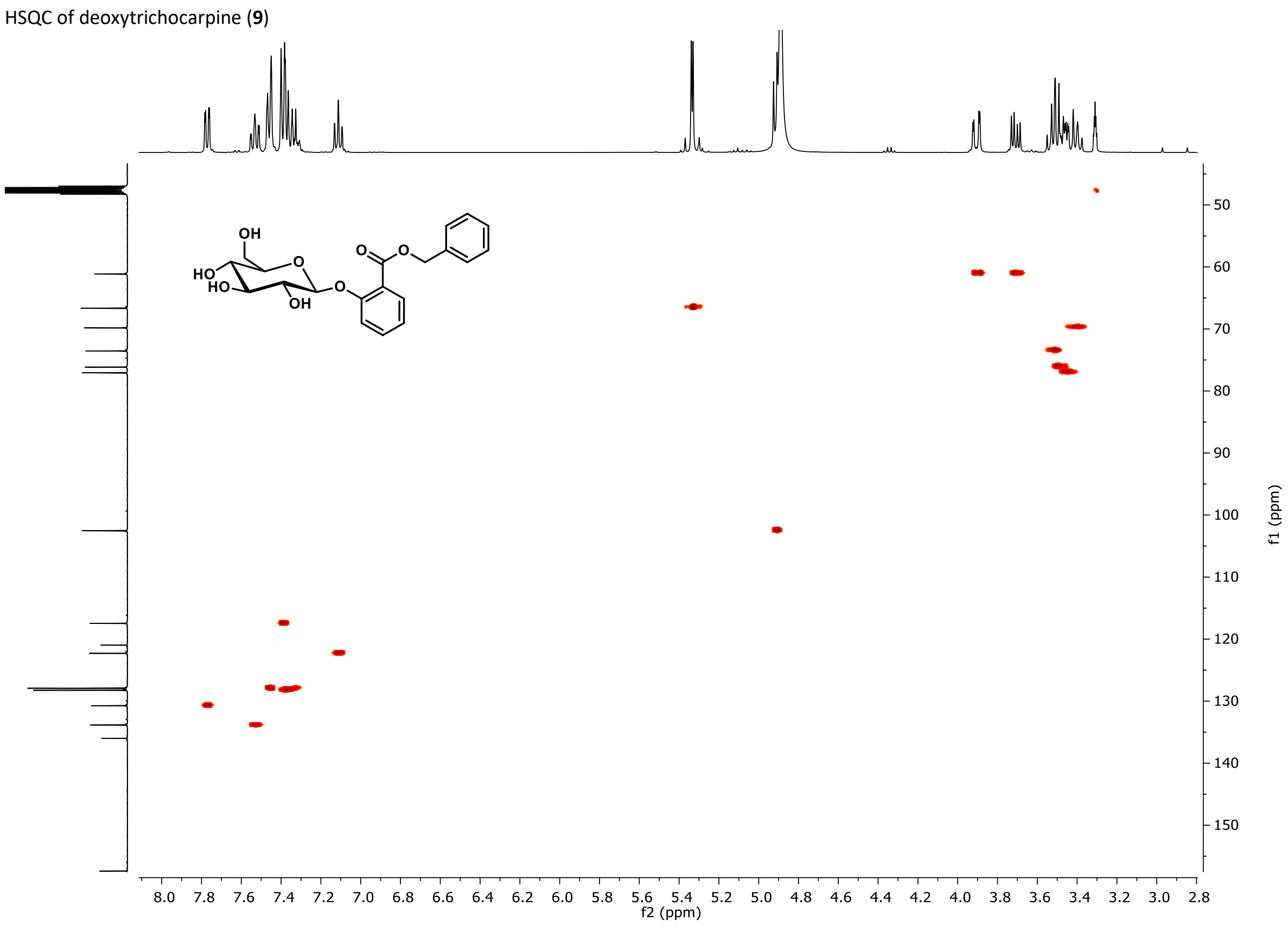


ATR-FTIR and UV of deoxytrichocarpine (9)
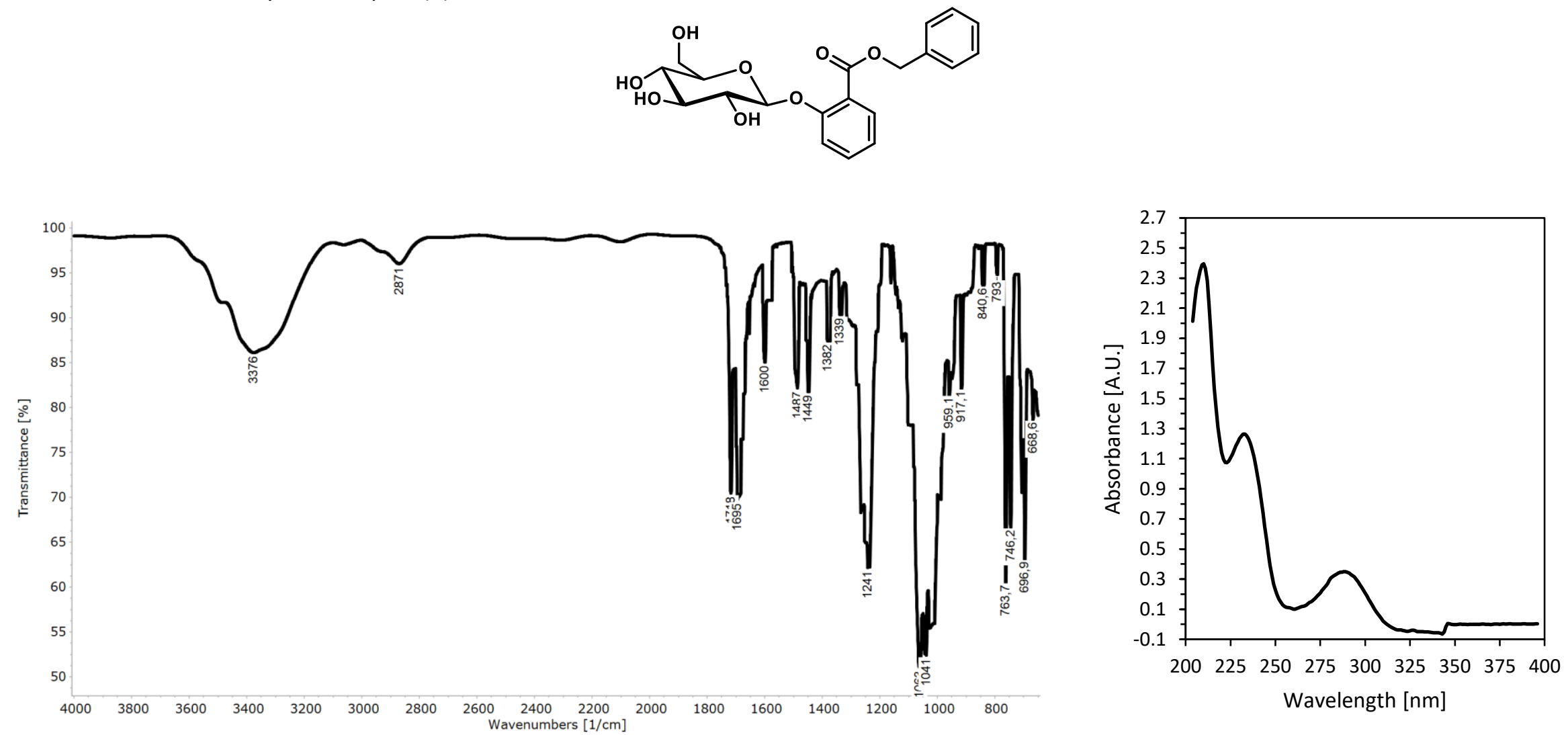


\section{5-Bromo-2-methoxybenzoic acid (12)}

${ }^{1} \mathrm{H}$ NMR (400 MHz, $\mathrm{CDCl}_{3}$ ) of 5-bromo-2-methoxybenzoic acid (12)
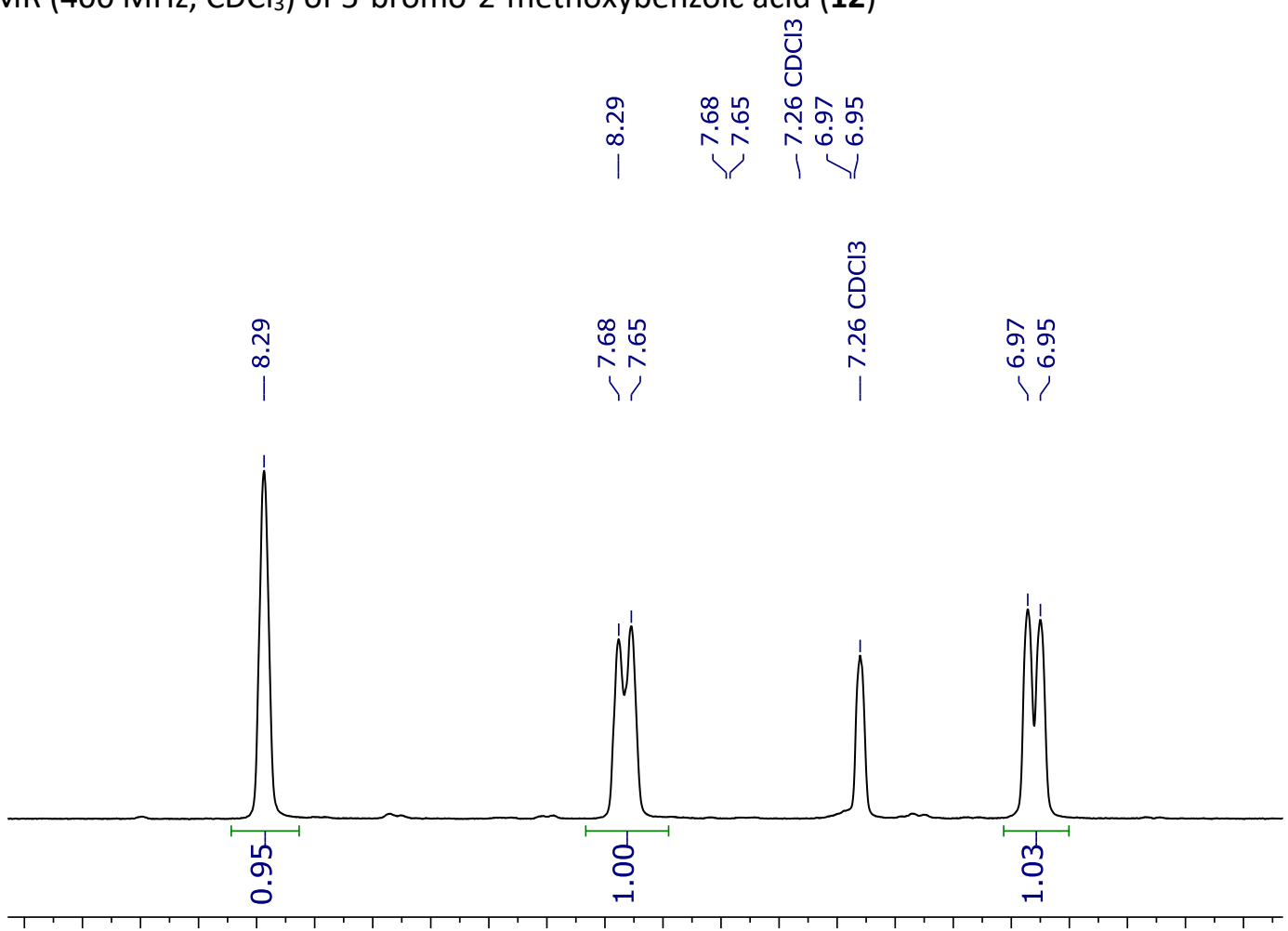

$\begin{array}{llllllllllllllllllllll}8.7 & 8.6 & 8.5 & 8.4 & 8.3 & 8.2 & 8.1 & 8.0 & 7.9 & 7.8 & 7.7 & 7.6 & 7.5 & 7.4 & 7.3 & 7.2 & 7.1 & 7.0 & 6.9 & 6.8 & 6.7 & 6.6\end{array}$ f1 (ppm)

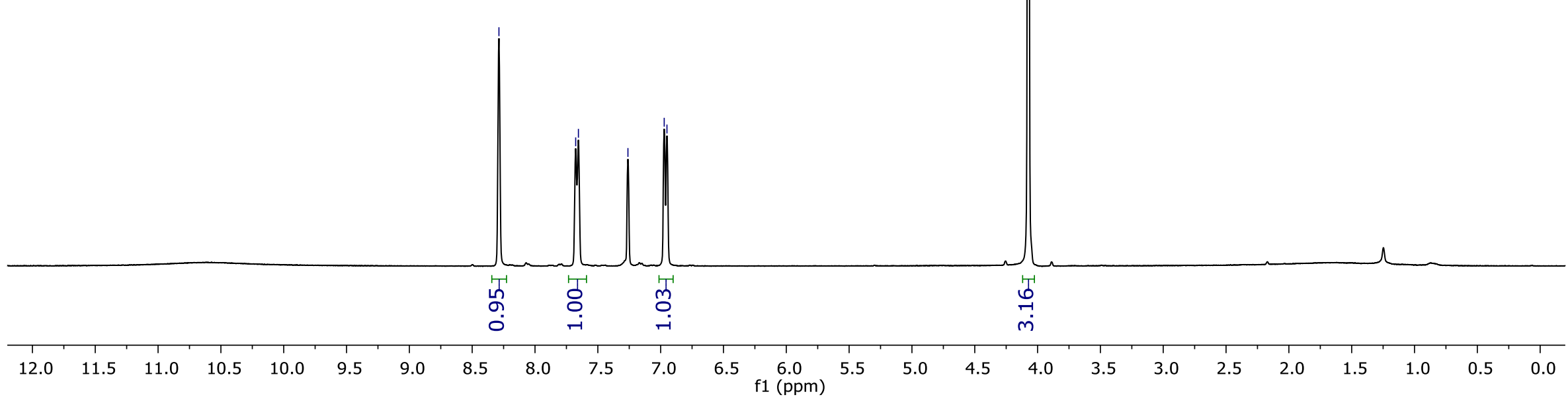


${ }^{13} \mathrm{C} \mathrm{NMR} \mathrm{(101} \mathrm{MHz,} \mathrm{CDCl}$ ) of 5-bromo-2-methoxybenzoic acid (12)

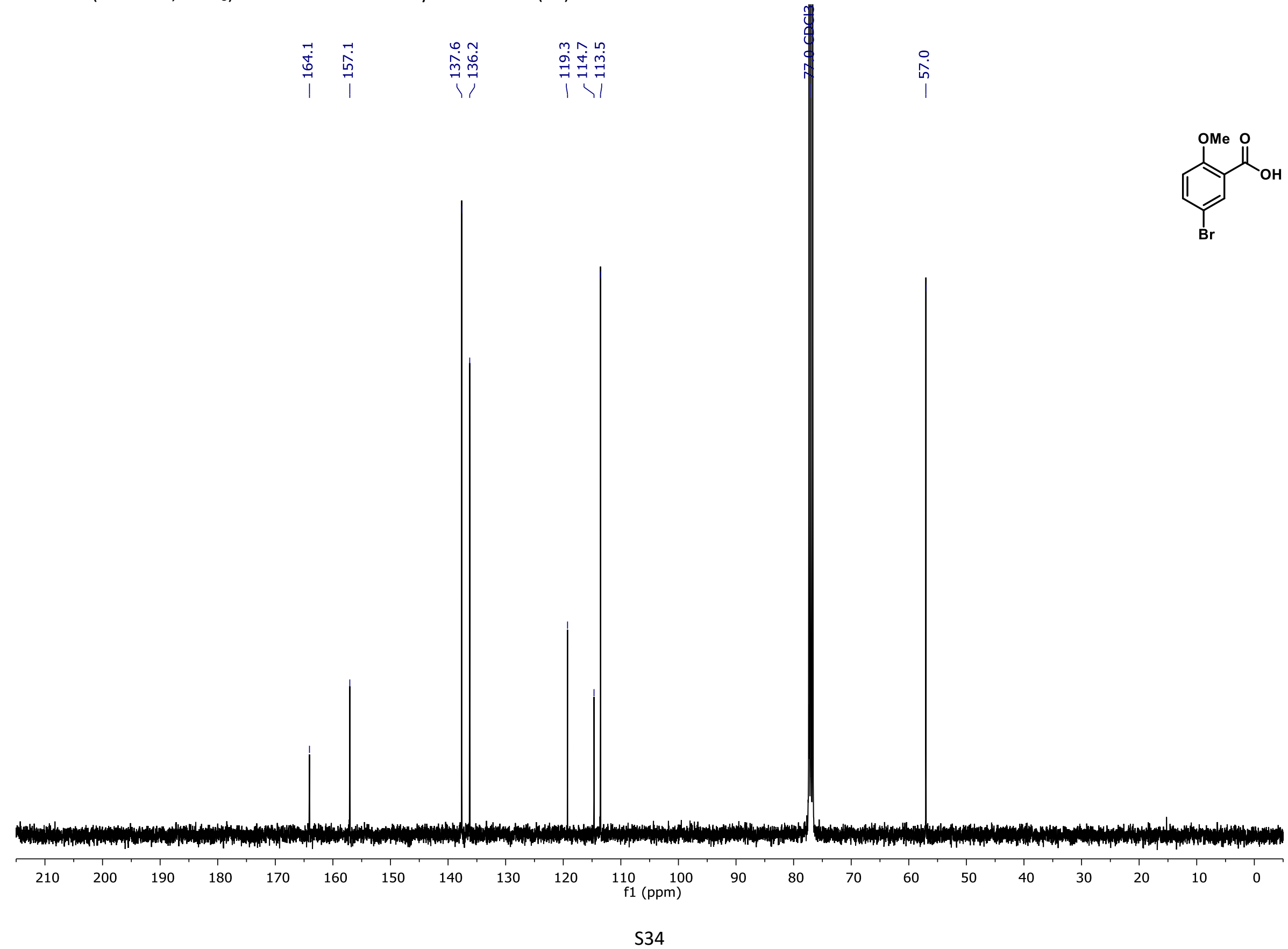



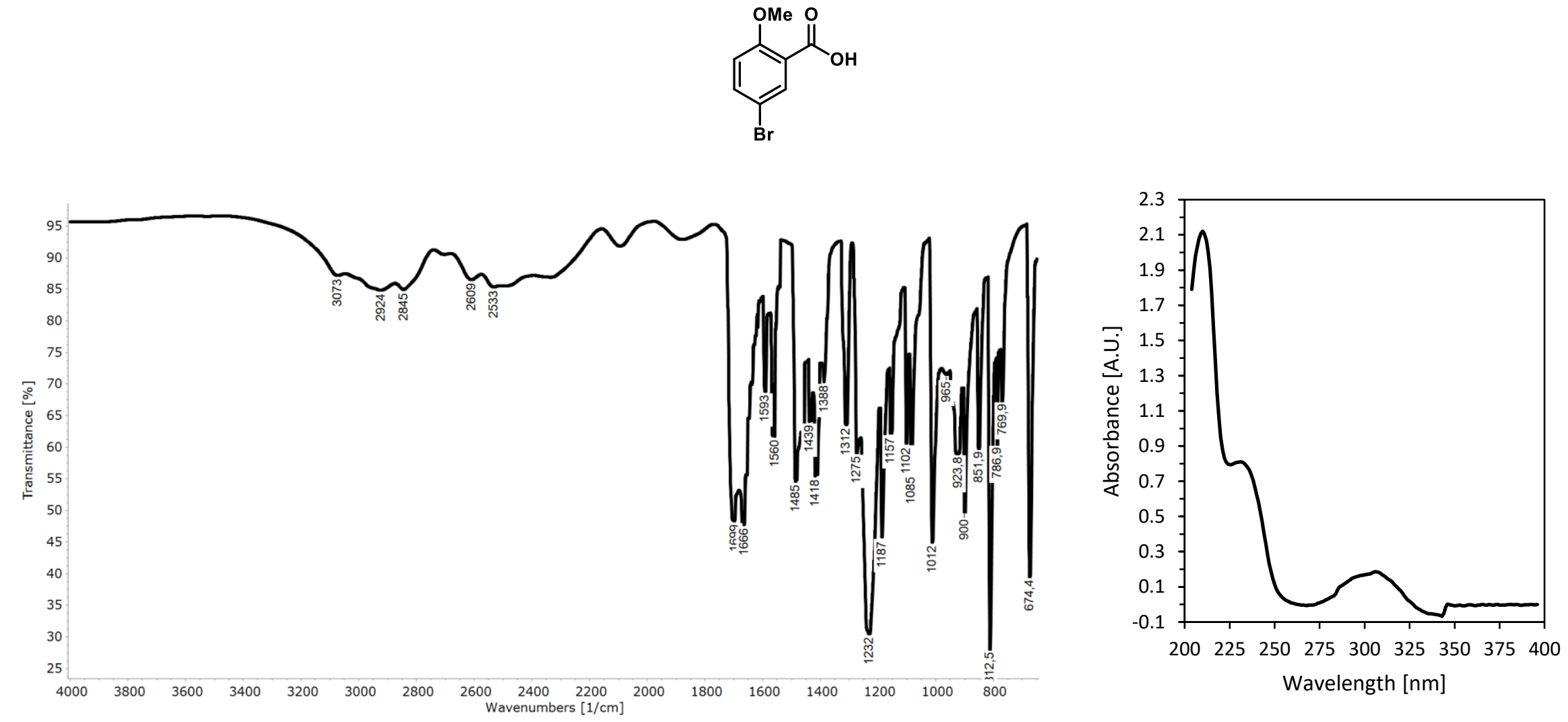


\section{5-Hydroxy-2-methoxybenzoic acid (10)}

${ }^{1} \mathrm{H}$ NMR $\left(400 \mathrm{MHz}\right.$, acetone- $\left.d_{6}\right)$ of 5-hydroxy-2-methoxybenzoic acid (10)

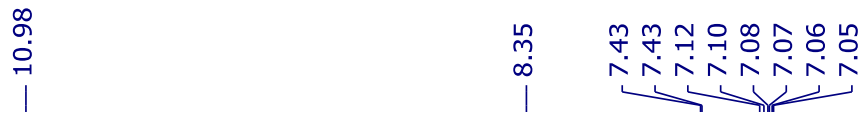

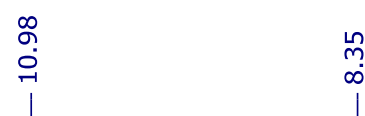
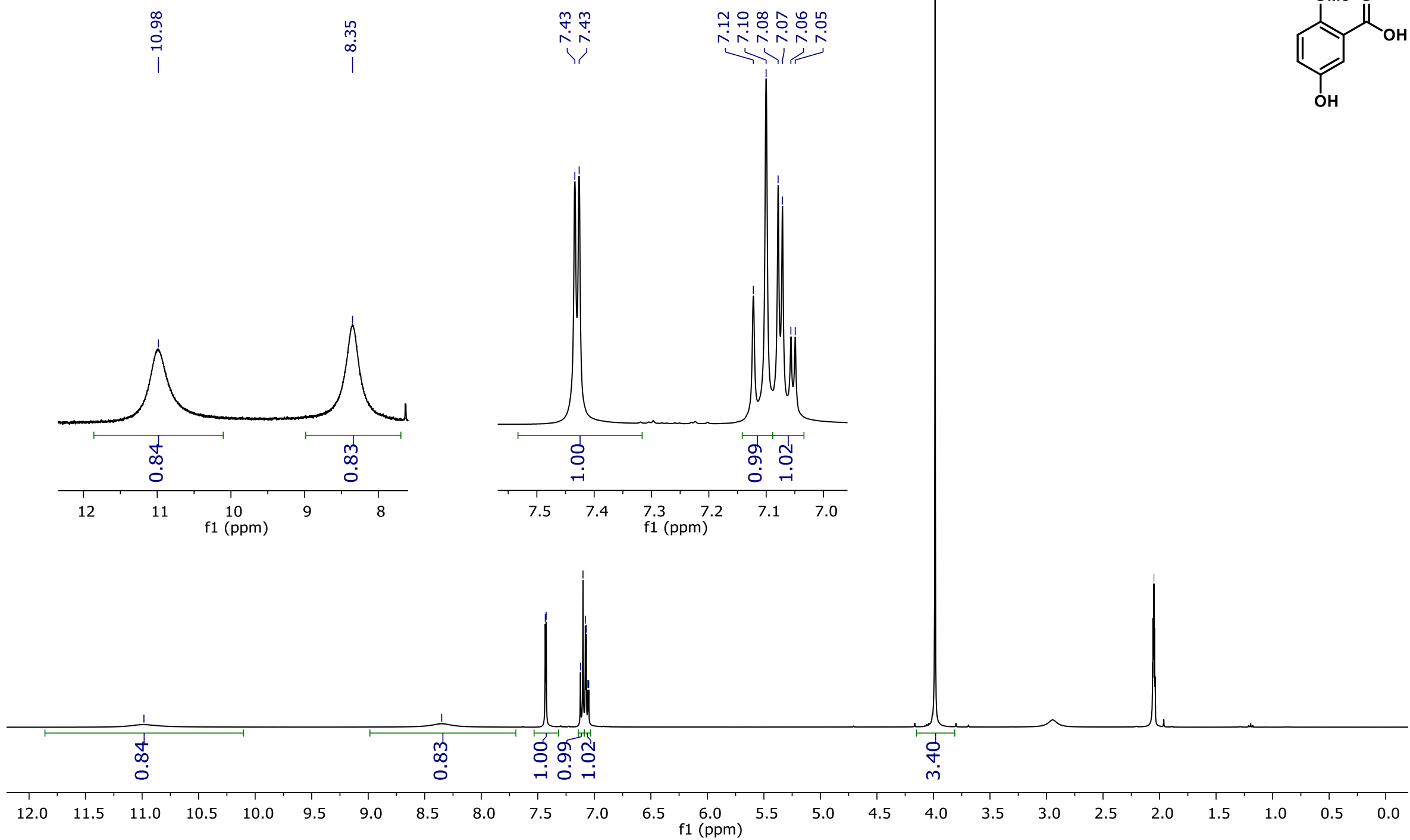


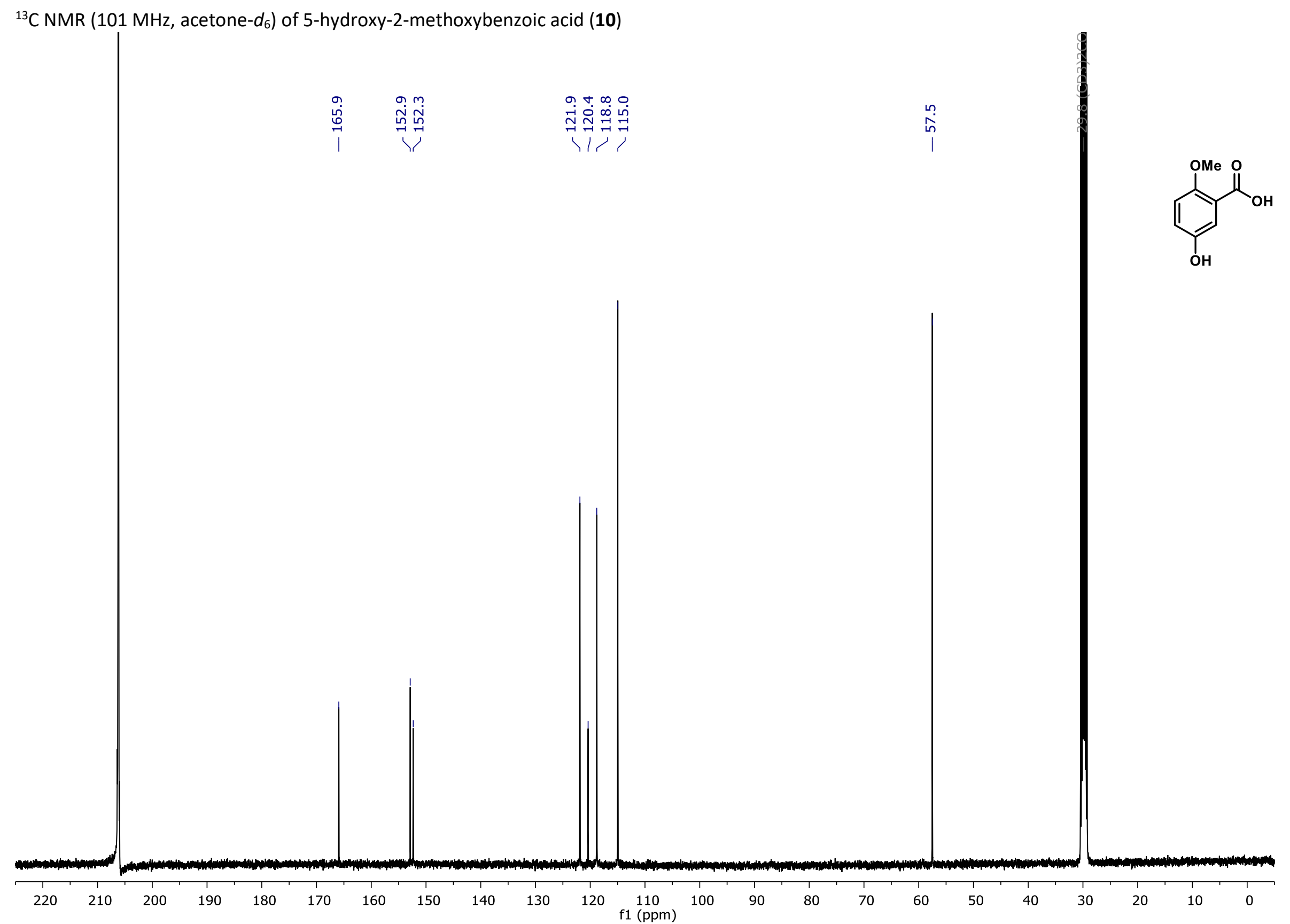



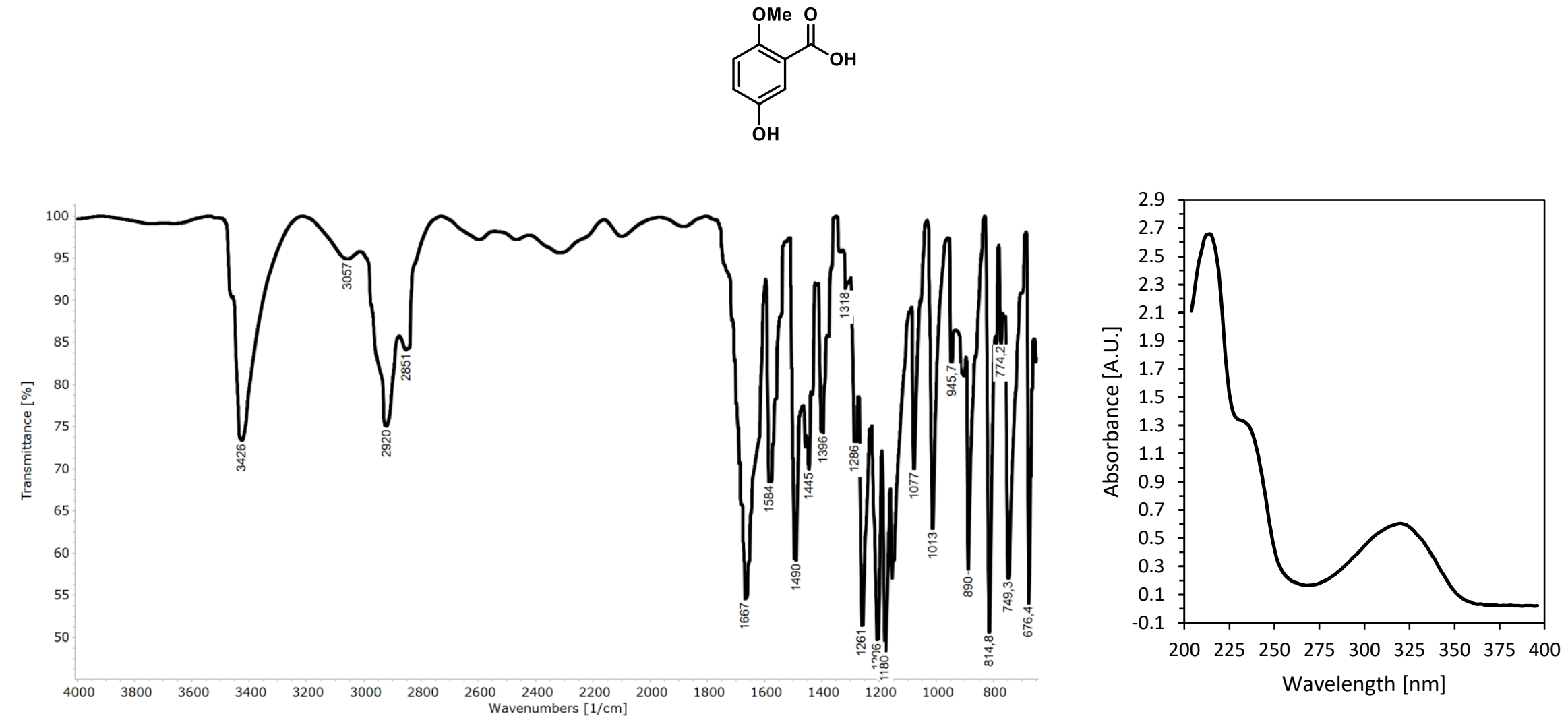


\section{Benzyl 5-acetoxy-2-hydroxybenzoate (13)}

${ }^{1} \mathrm{H}$ NMR (400 MHz, $\mathrm{CDCl}_{3}$ ) of benzyl 5-acetoxy-2-hydroxybenzoate (13)
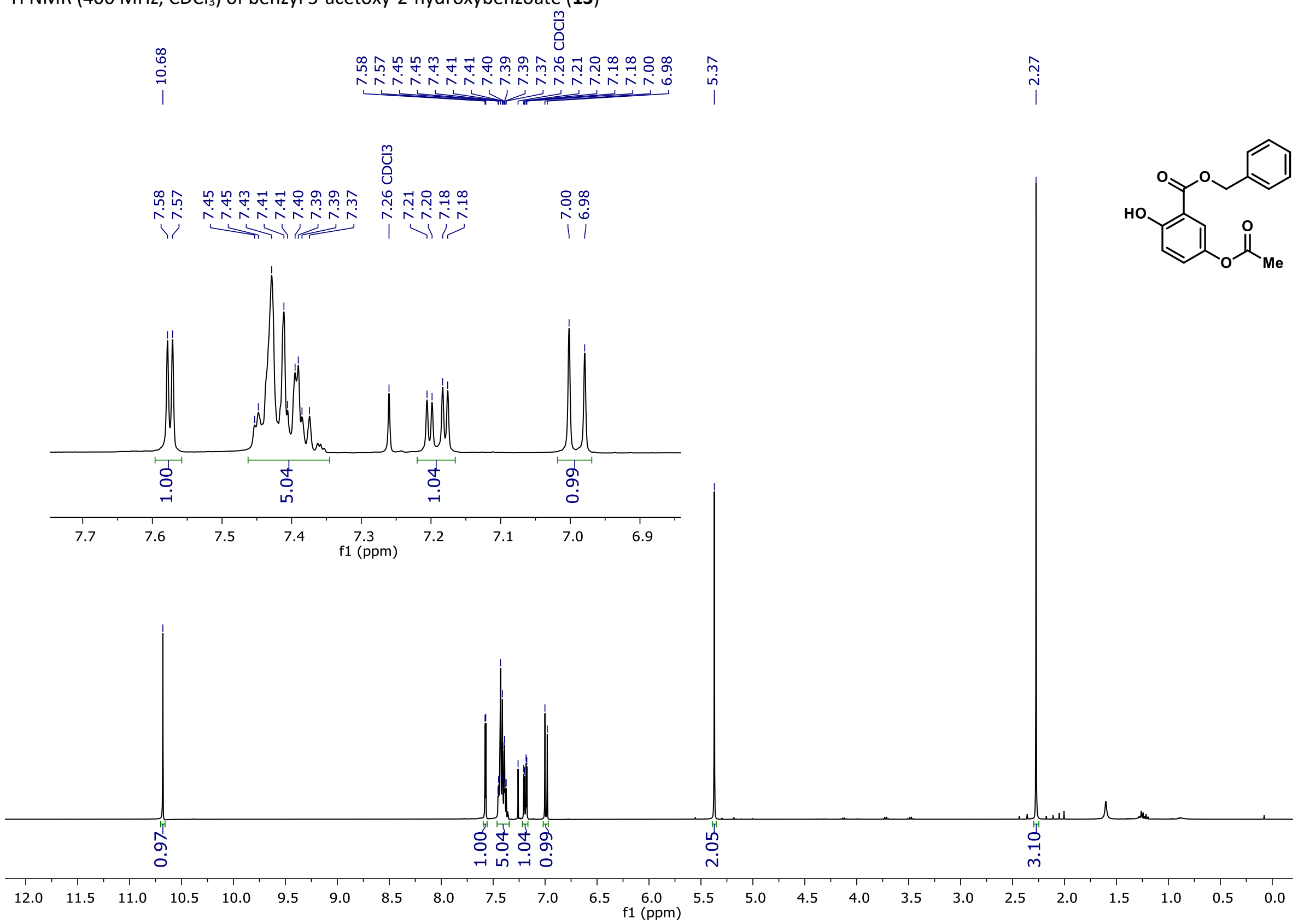
${ }^{13} \mathrm{C}$ NMR (101 MHz, $\mathrm{CDCl}_{3}$ ) of benzyl 5-acetoxy-2-hydroxybenzoate (13)

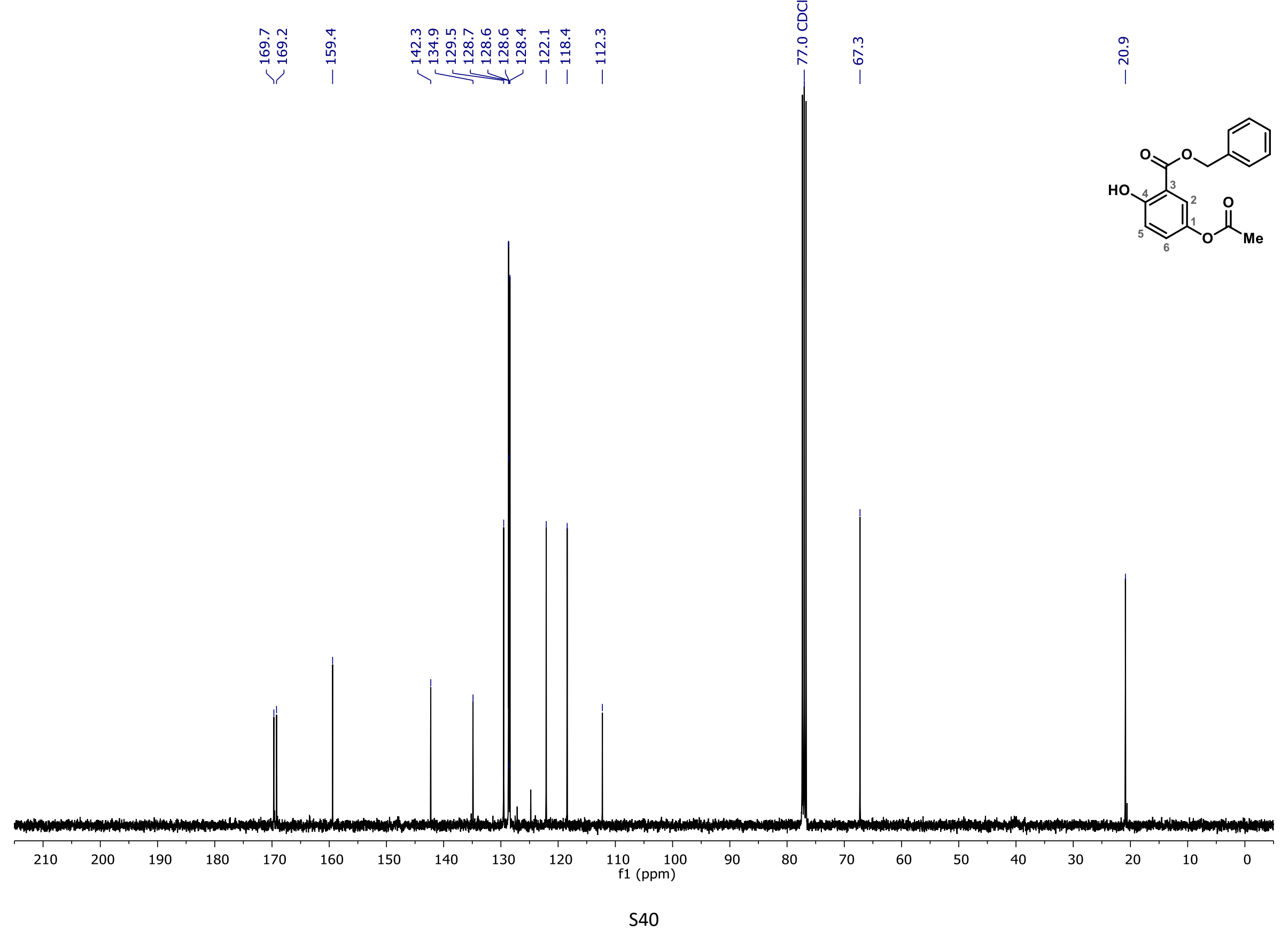


HMBC of benzyl 5-acetoxy-2-hydroxybenzoate (13)

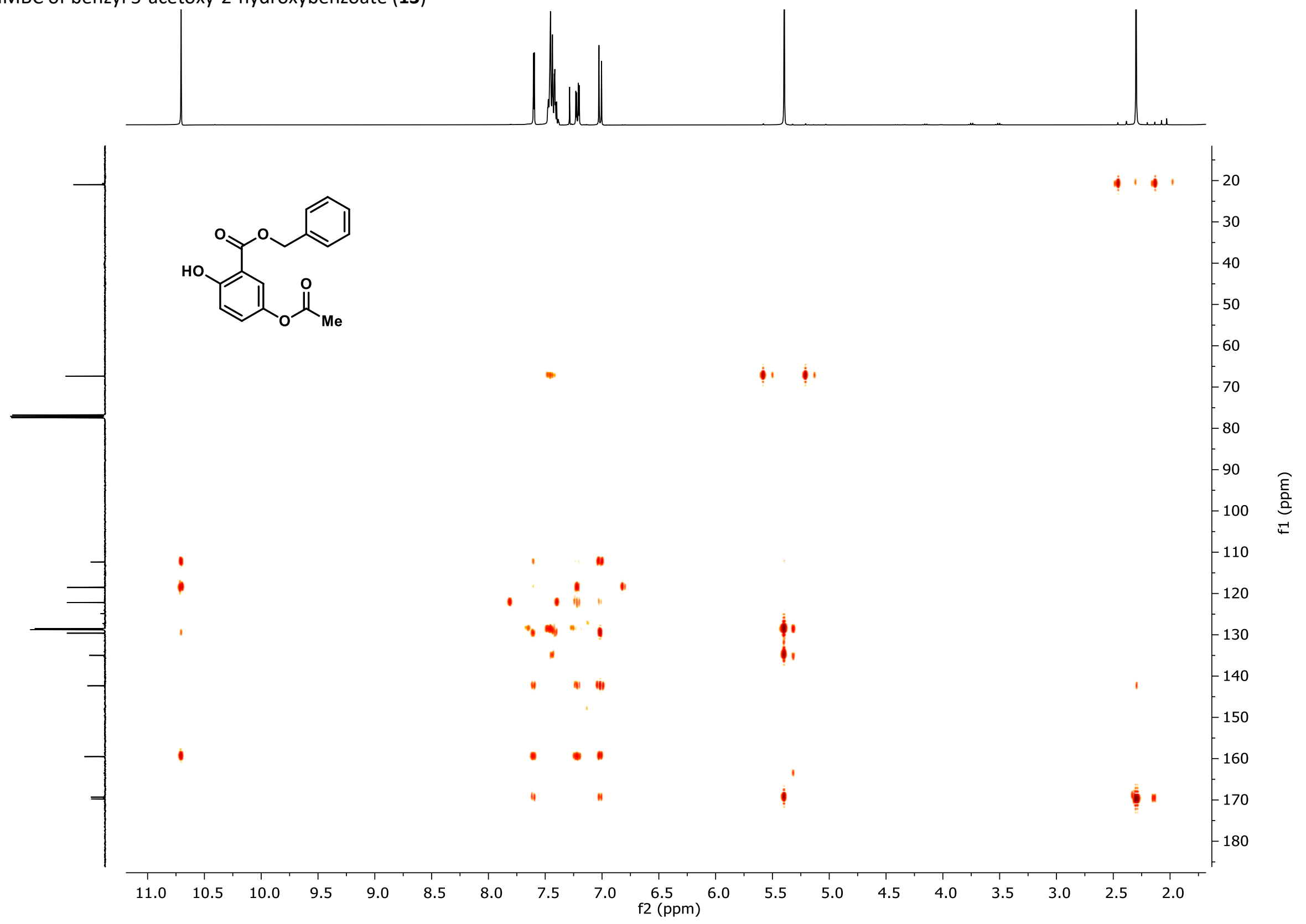




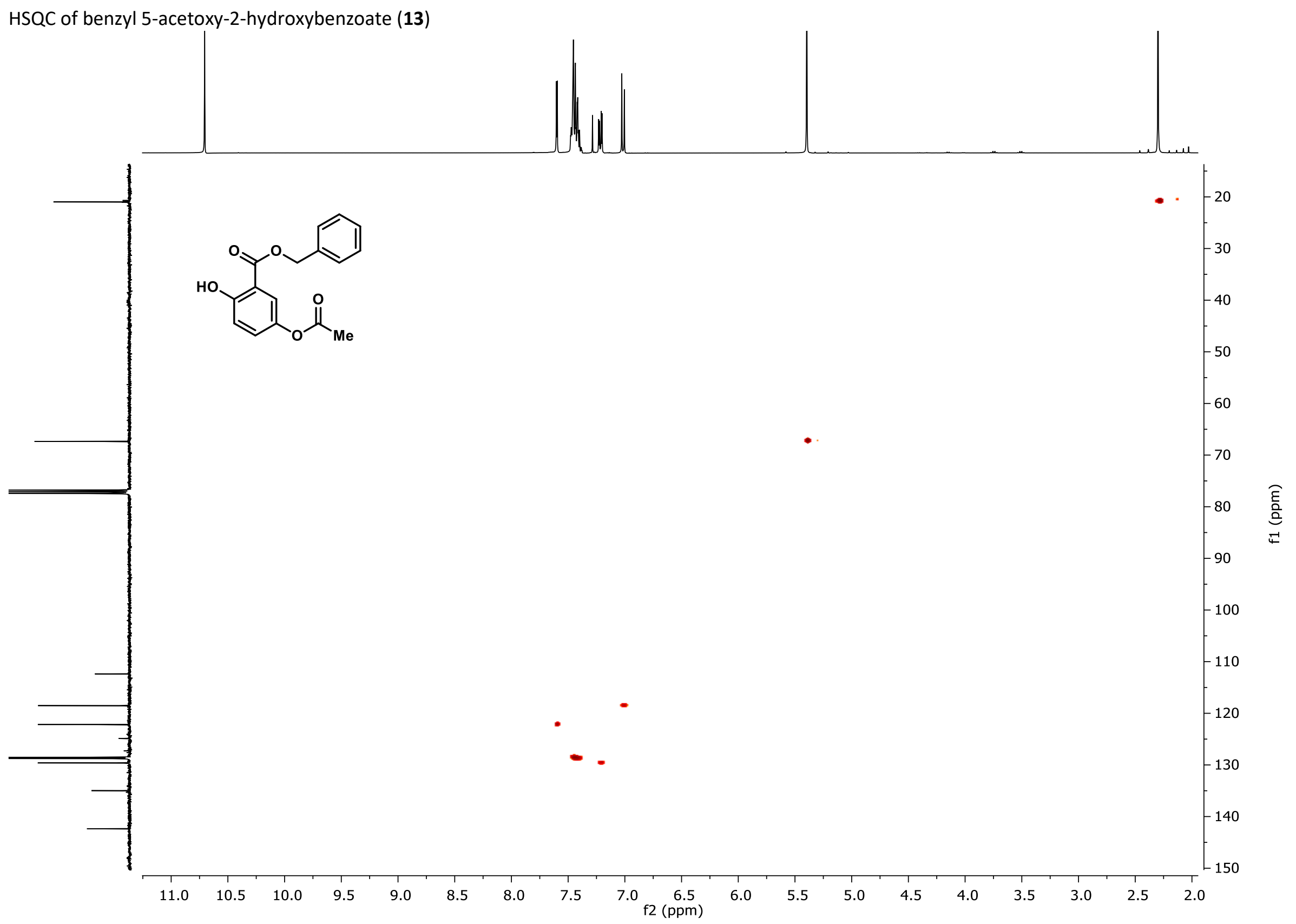



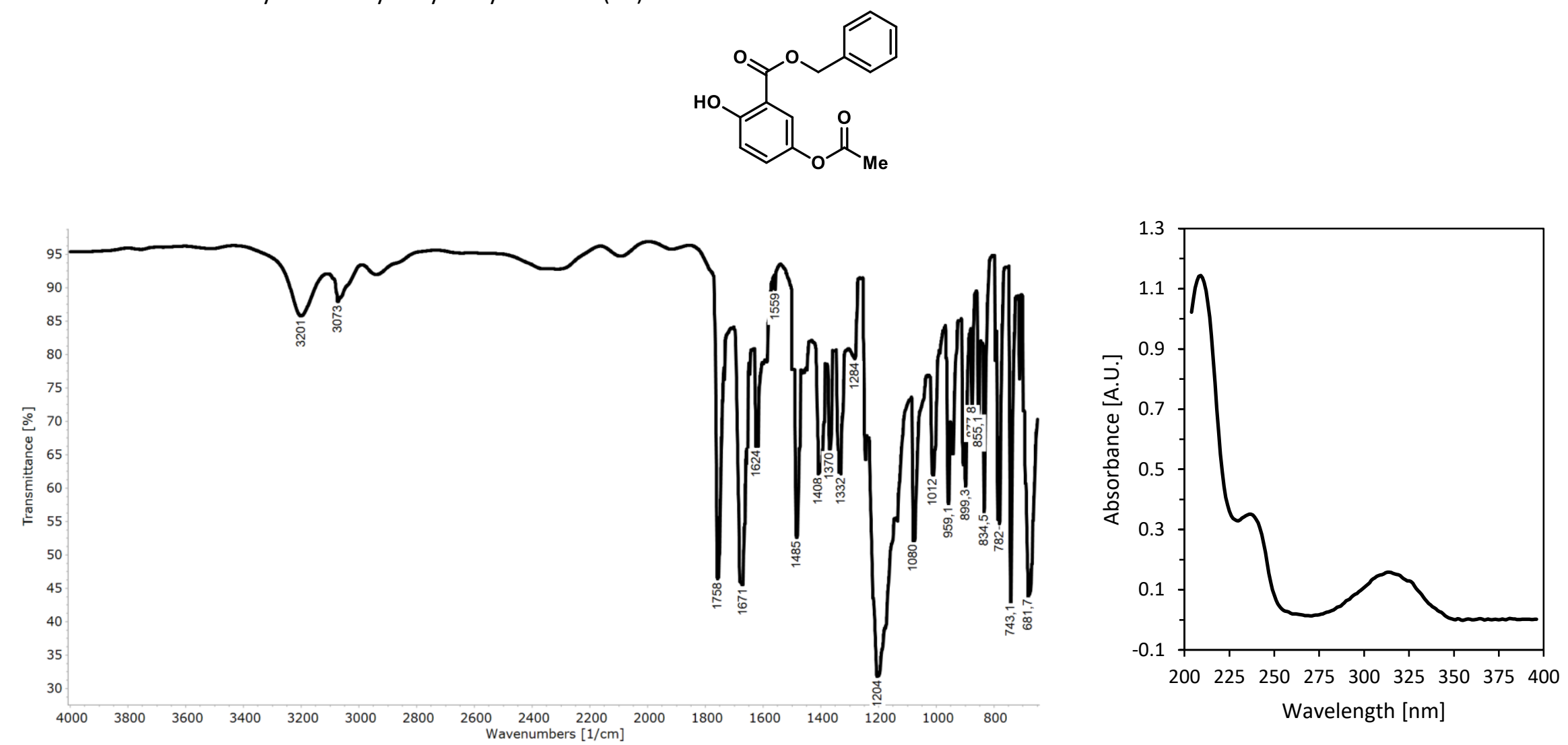


\section{Benzyl 5-hydroxy-2-methoxybenzoate (14)}

${ }^{1} \mathrm{H}$ NMR (400 MHz, $\mathrm{CDCl}_{3}$ ) of benzyl 5-hydroxy-2-methoxybenzoate (14)
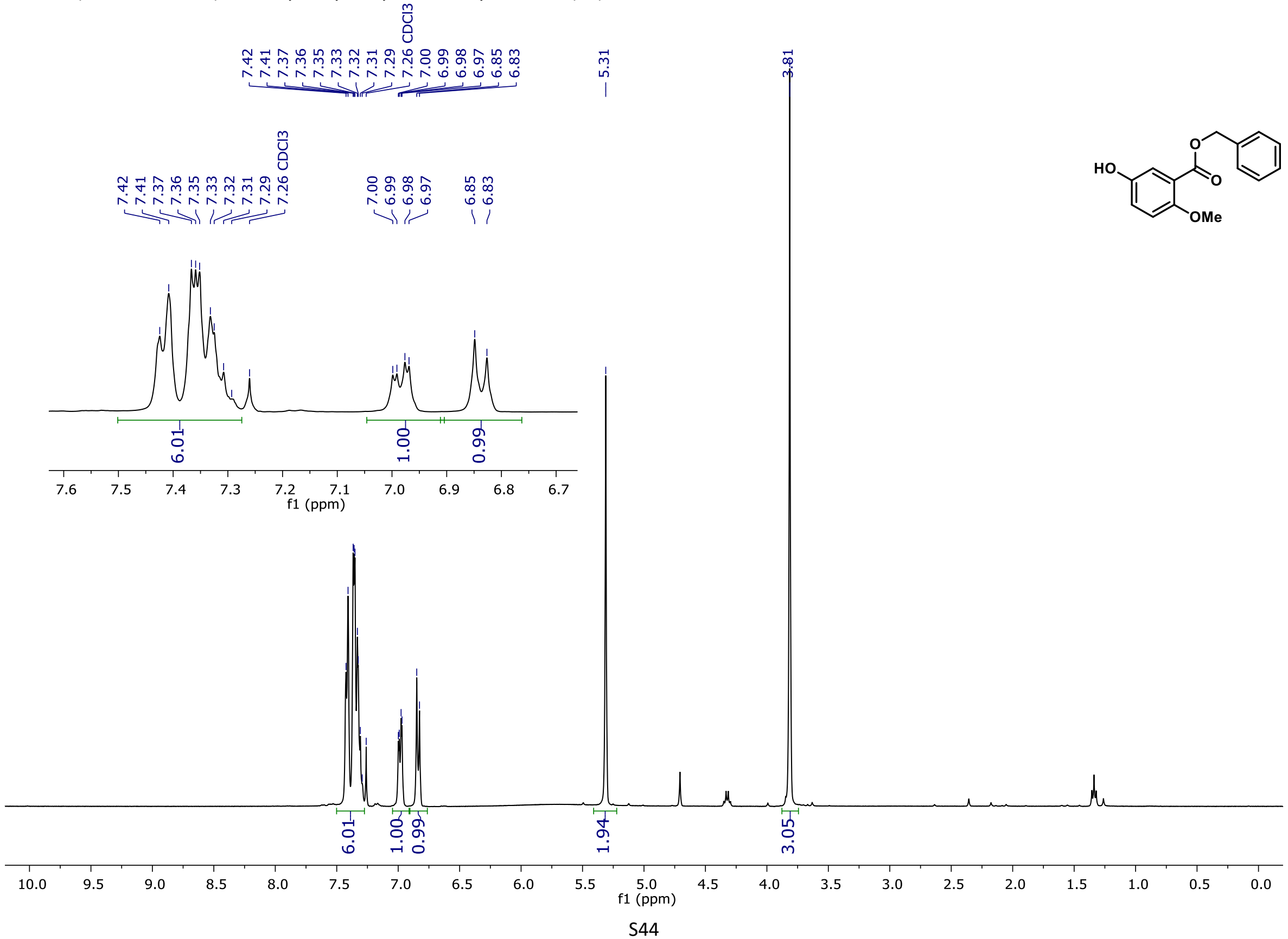
${ }^{13} \mathrm{C} \mathrm{NMR} \mathrm{(101} \mathrm{MHz,} \mathrm{CDCl}_{3}$ ) of benzyl 5-hydroxy-2-methoxybenzoate (14)

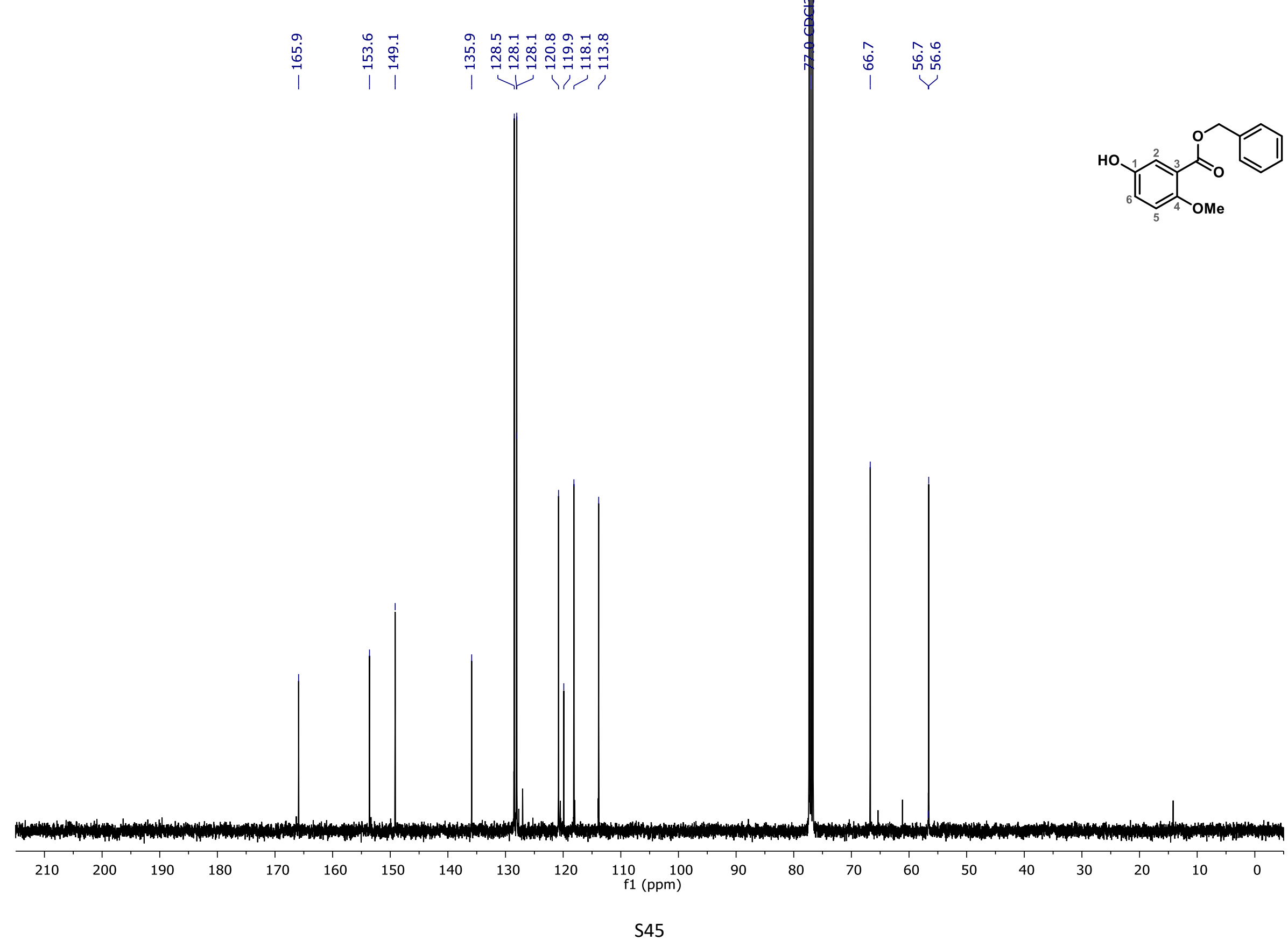


HMBC of benzyl 5-hydroxy-2-methoxybenzoate (14)

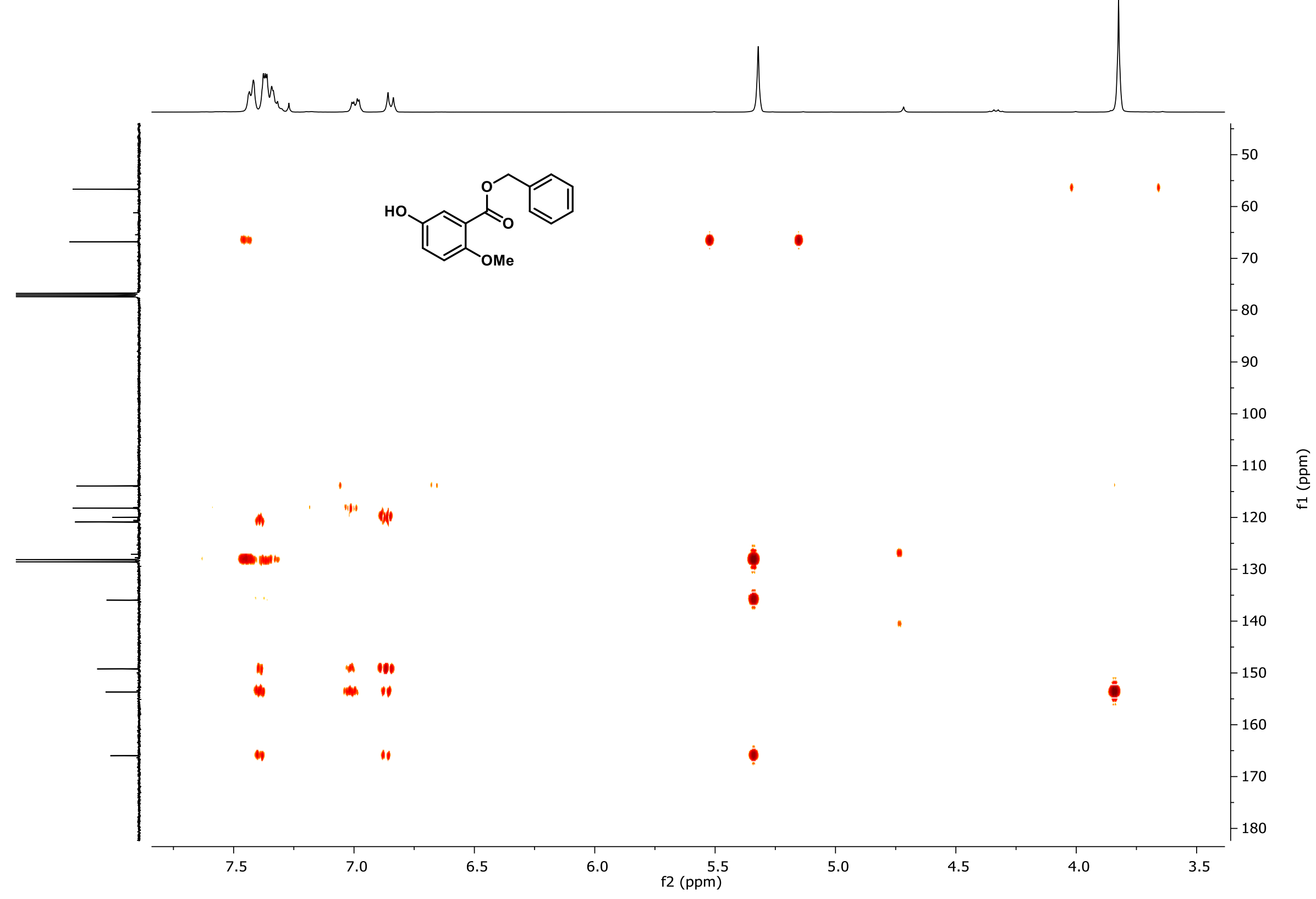


HSQC of benzyl 5-hydroxy-2-methoxybenzoate (14)

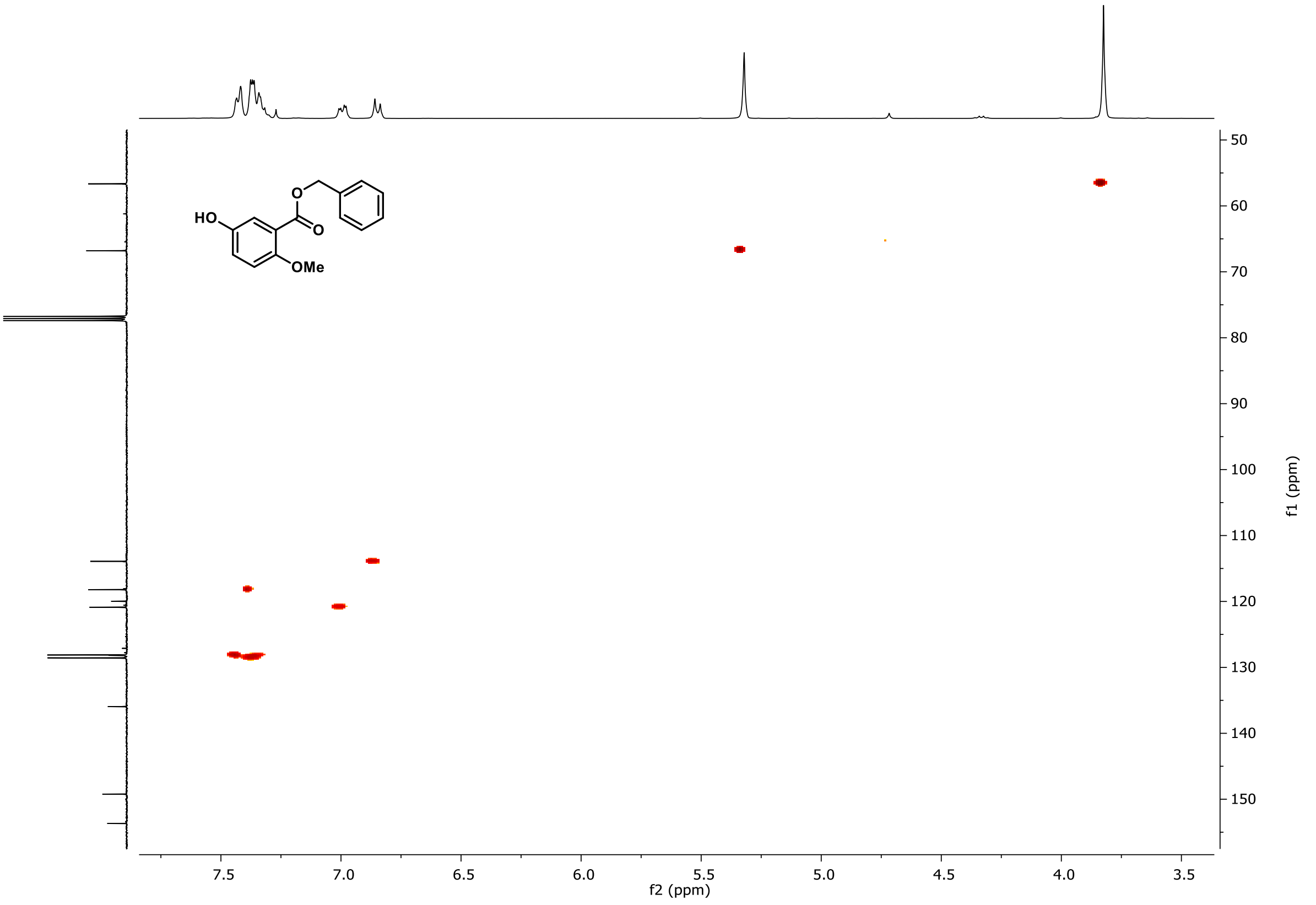


ATR-FTIR of benzyl 5-hydroxy-2-methoxybenzoate (14)
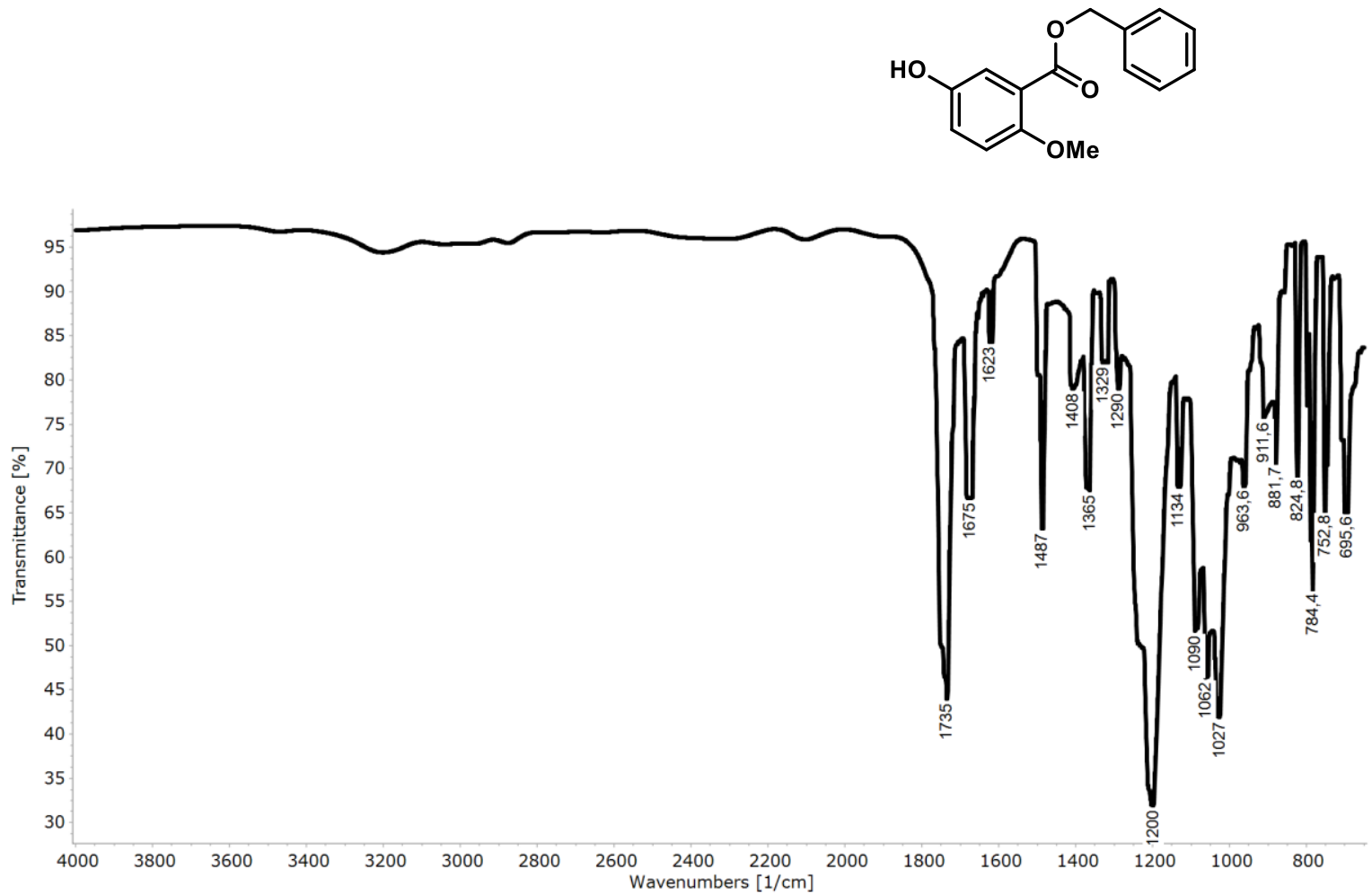


\section{Isotrichocarpine tetraacetate (15)}

${ }^{1} \mathrm{H}$ NMR (400 MHz, $\mathrm{CDCl}_{3}$ ) of isotrichocarpine tetraacetate (15)

บे

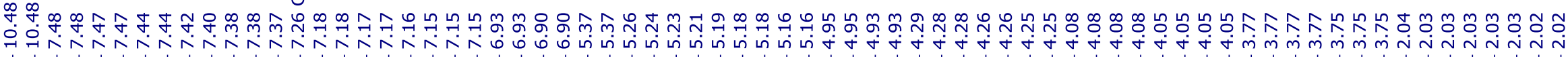

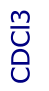

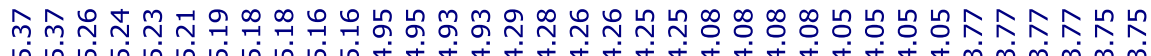

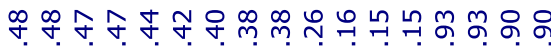

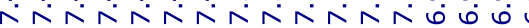

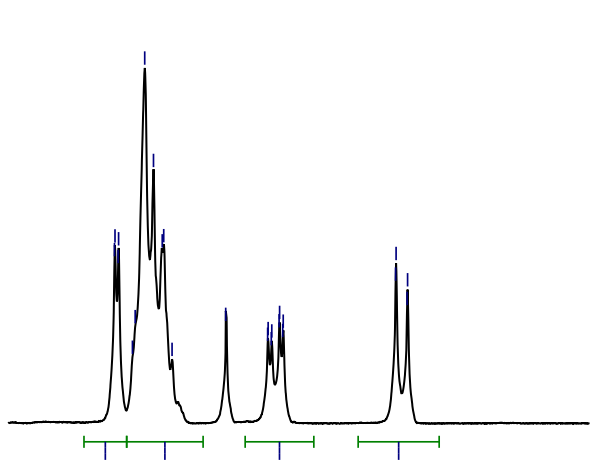

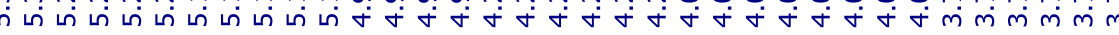
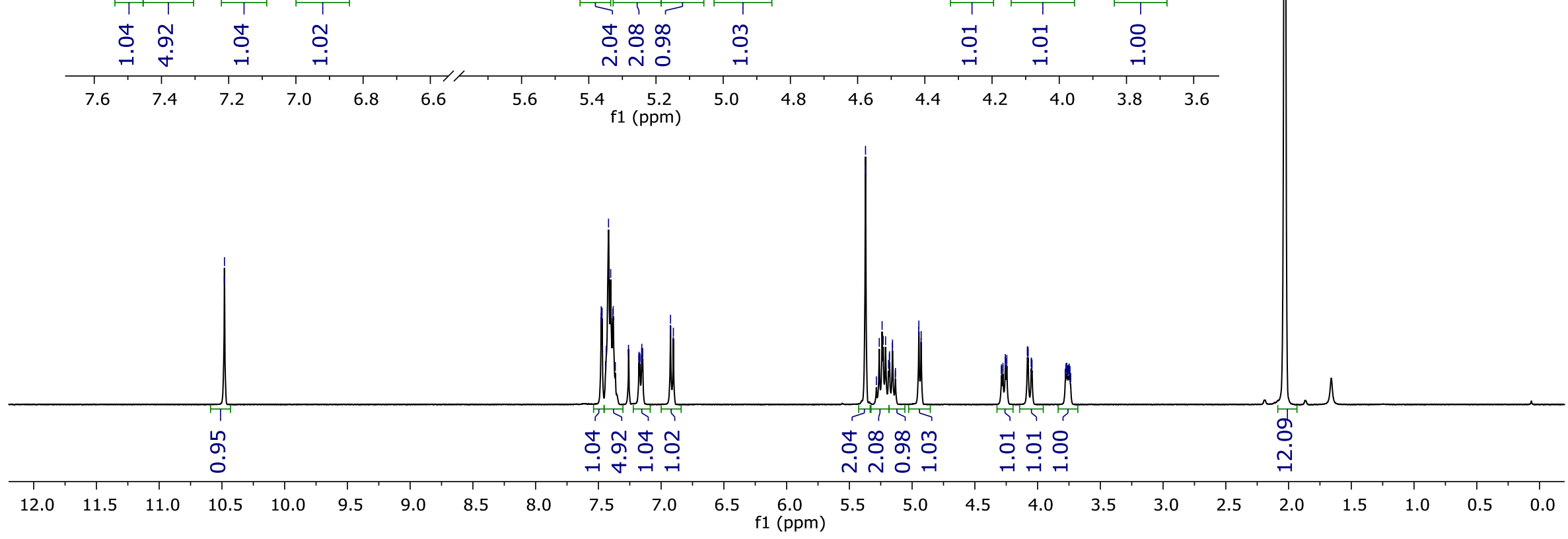


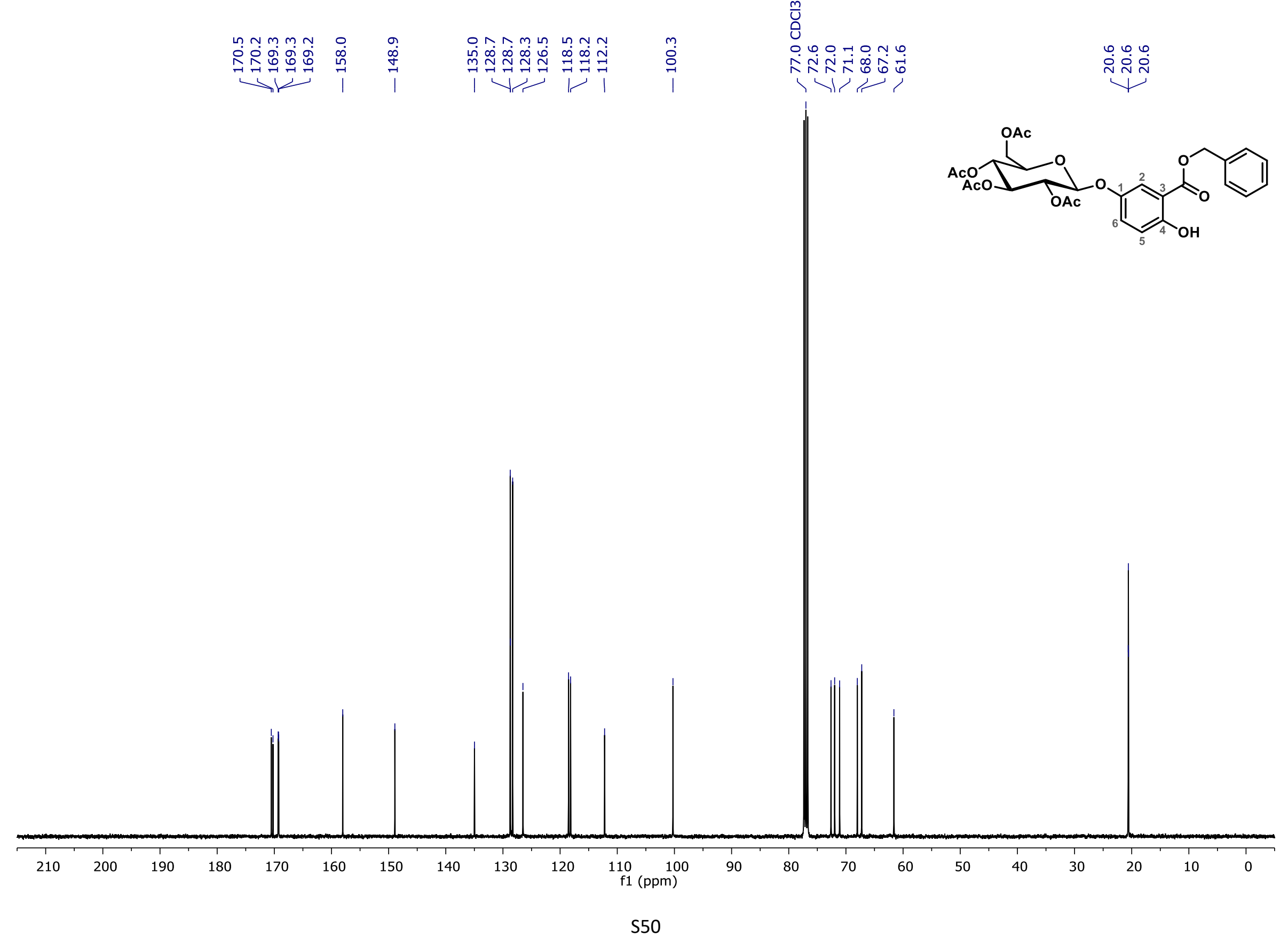


COSY of isotrichocarpine tetraacetate (15)

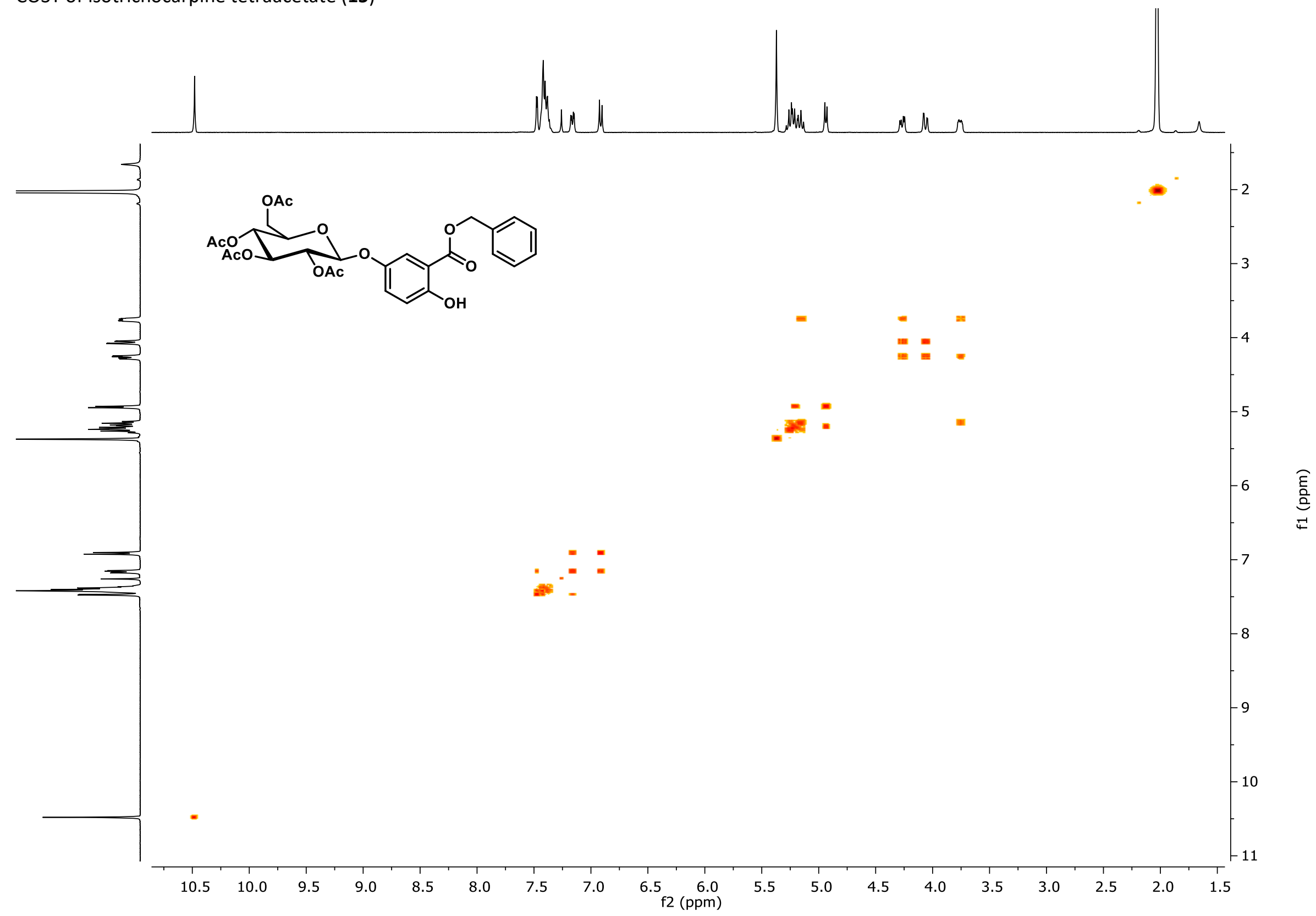


HSQC of isotrichocarpine tetraacetate (15)

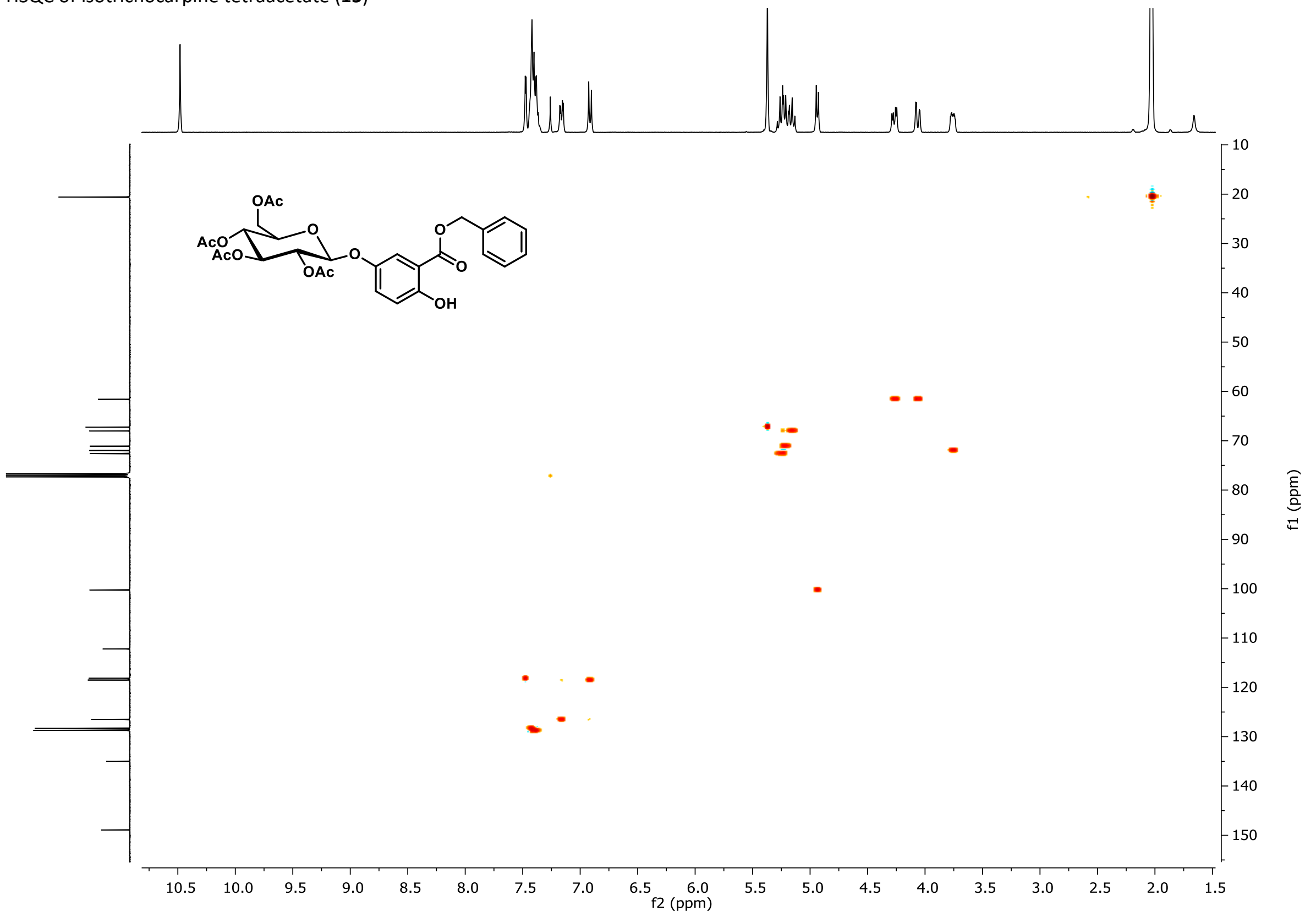


ATR-FTIR and UV of isotrichocarpine tetraacetate (15)
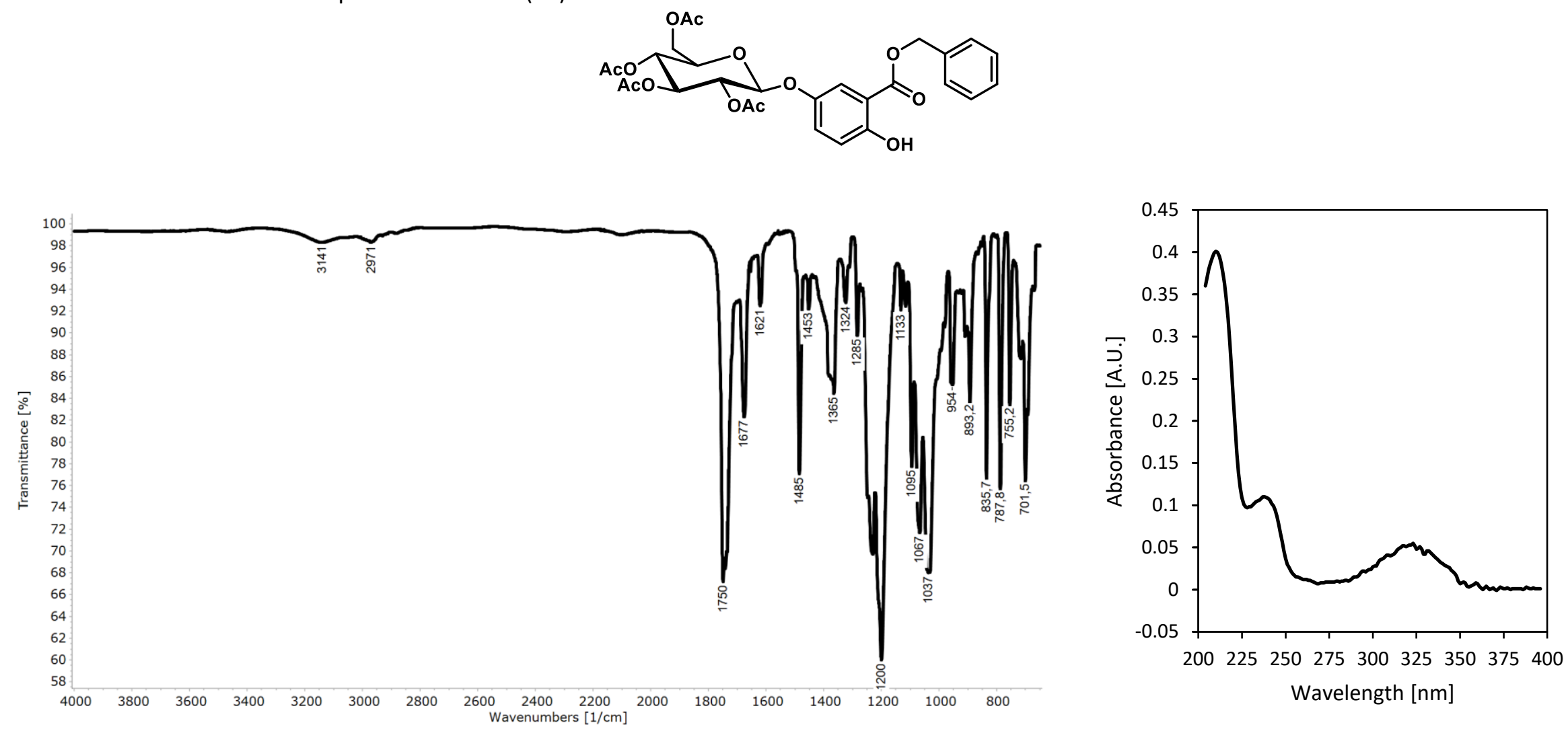


\section{Trichocarpine pentaacetate (16)}

${ }^{1} \mathrm{H} \mathrm{NMR}\left(400 \mathrm{MHz}, \mathrm{CDCl}_{3}\right)$ of trichocarpine pentaacetate (16)

$$
\frac{m}{\mathrm{U}}
$$

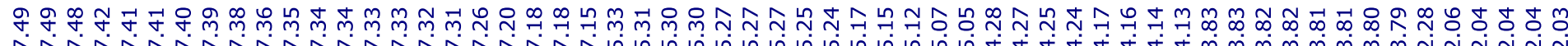

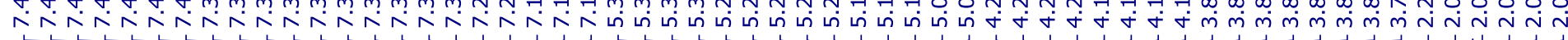

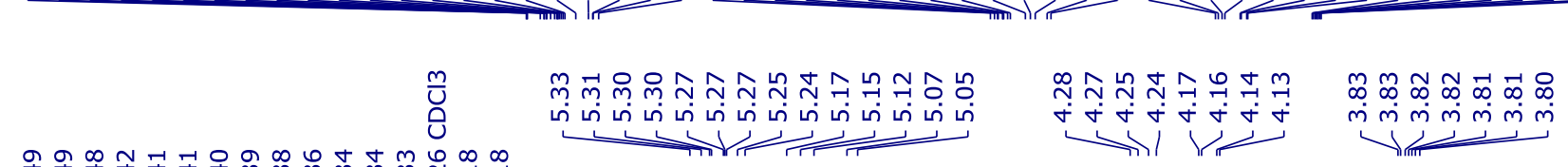

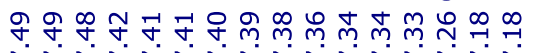

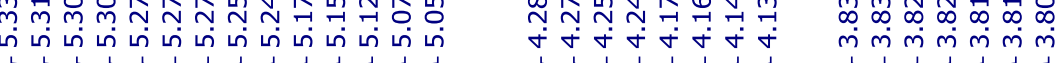

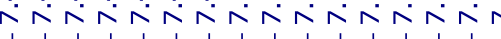
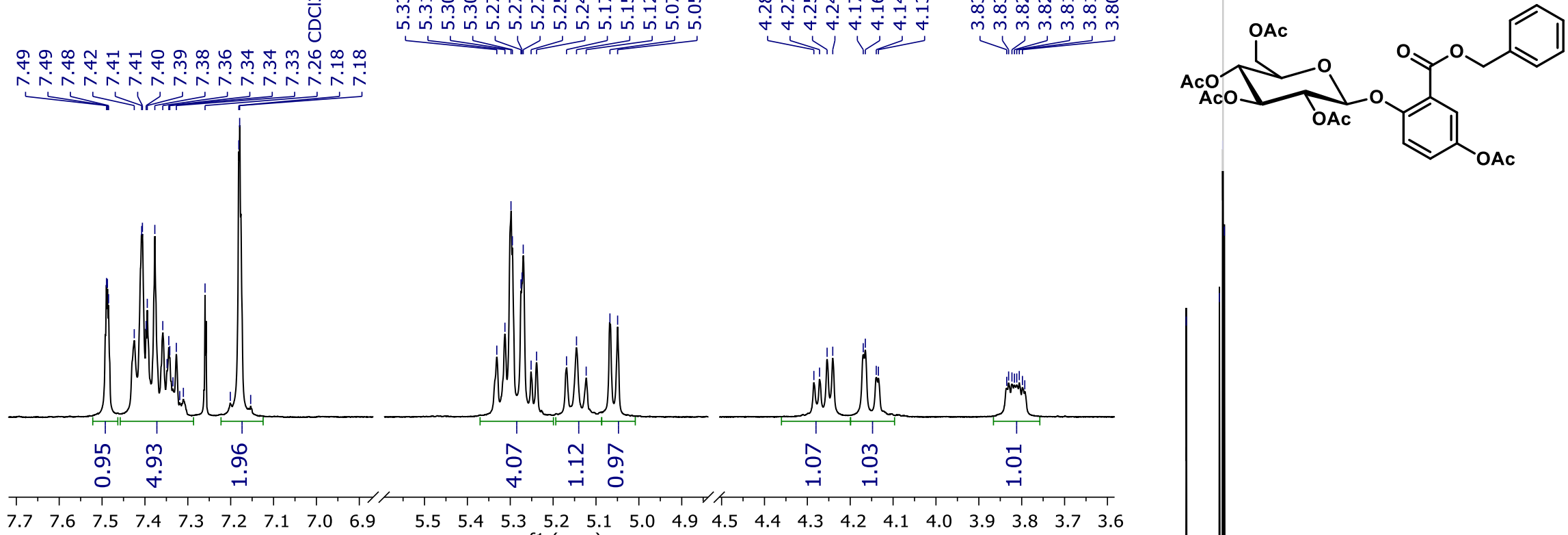
3 f1 (ppm)

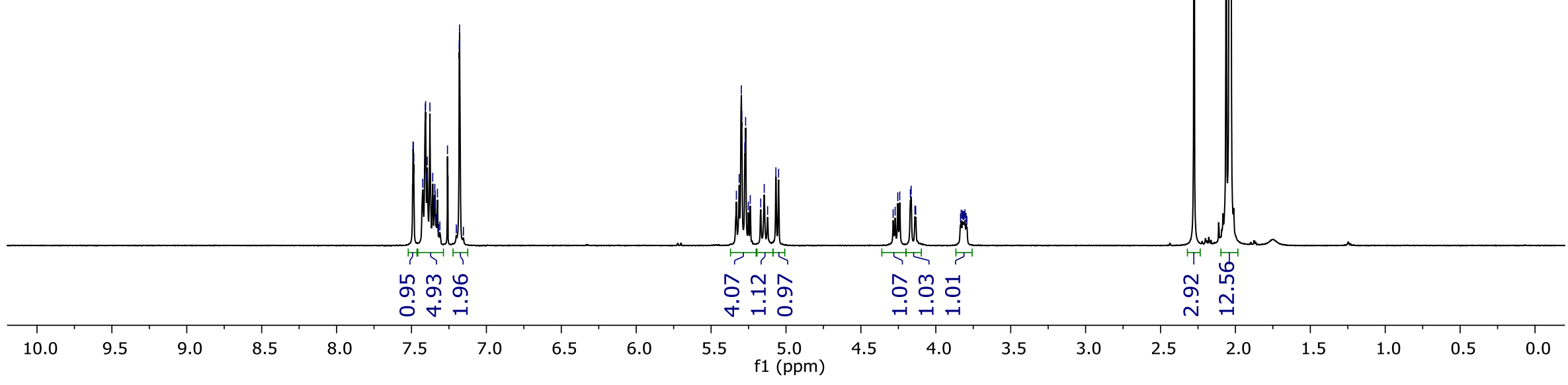


${ }^{13} \mathrm{C}$ NMR (101 MHz, $\mathrm{CDCl}_{3}$ ) of trichocarpine pentaacetate (16)

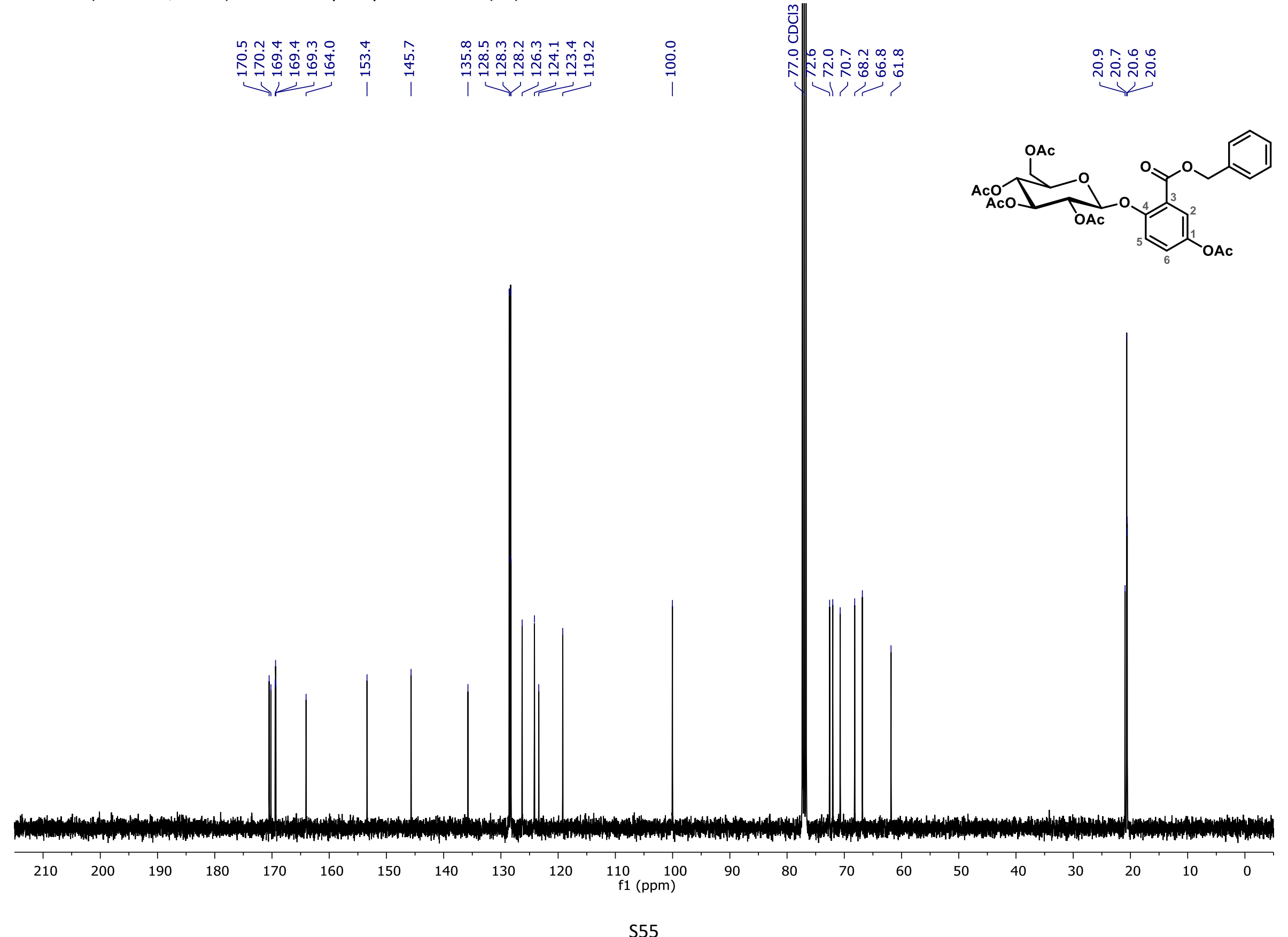




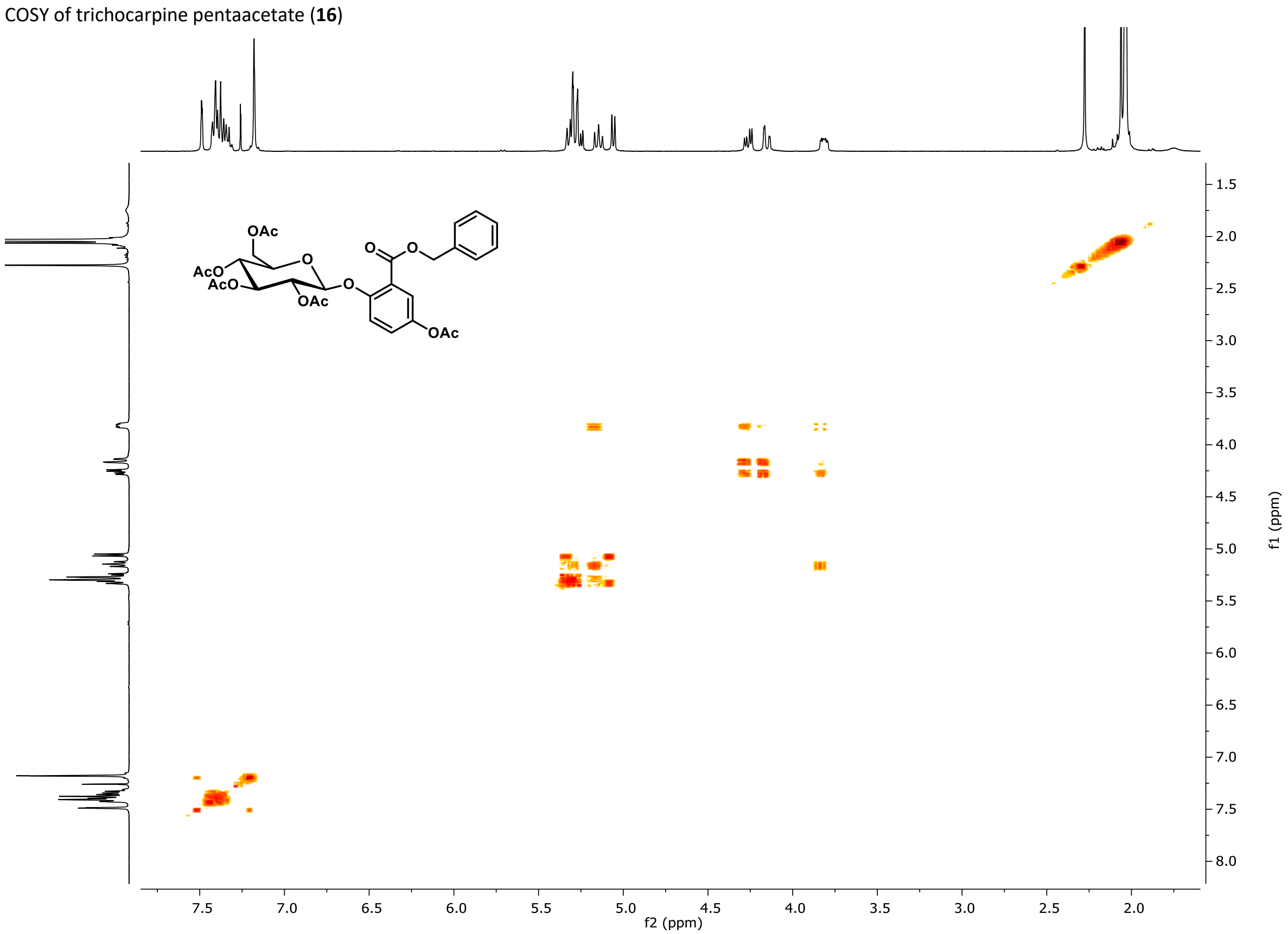


HSQC of trichocarpine pentaacetate (16)

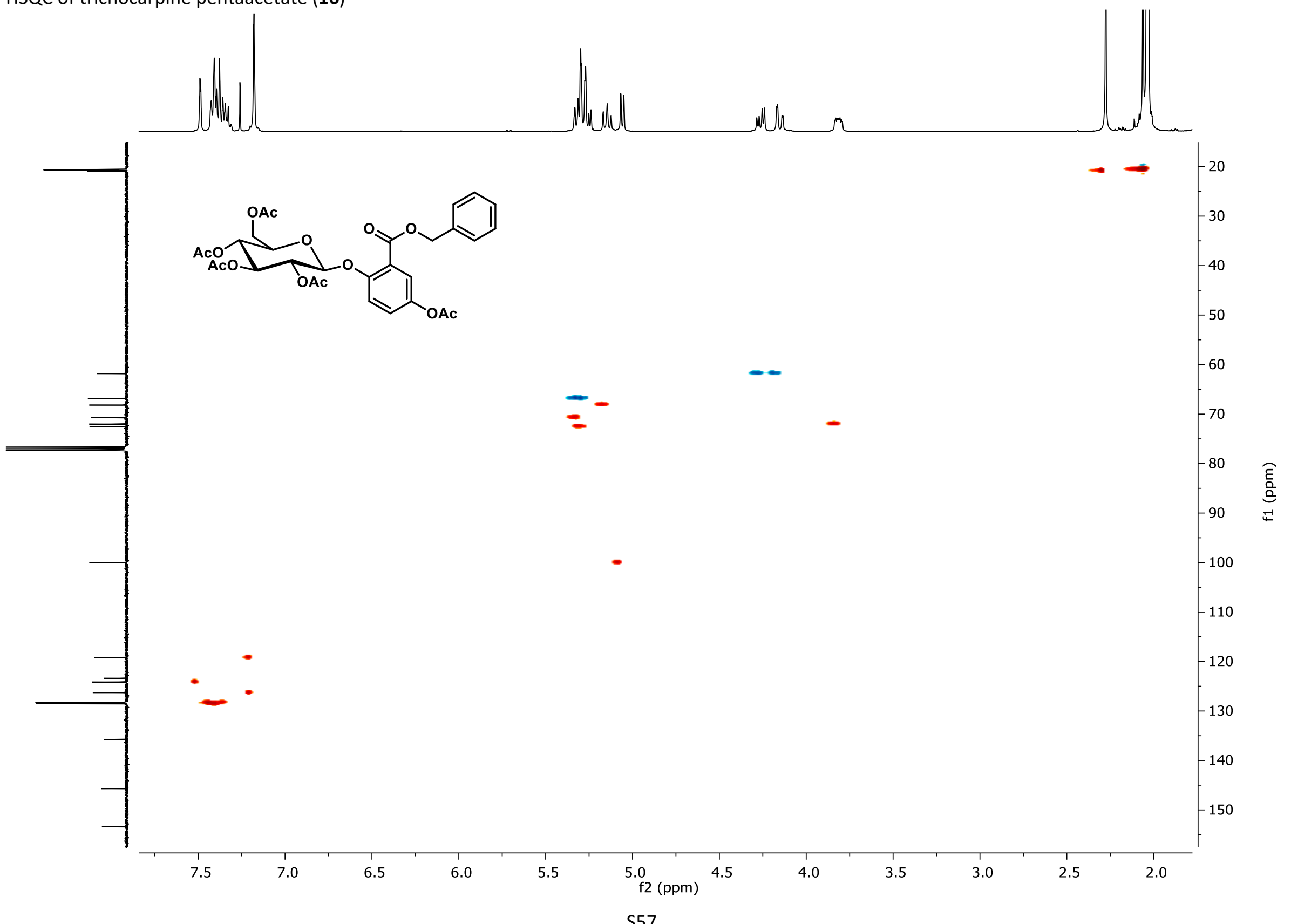


ATR-FTIR and UV of trichocarpine pentaacetate (16)
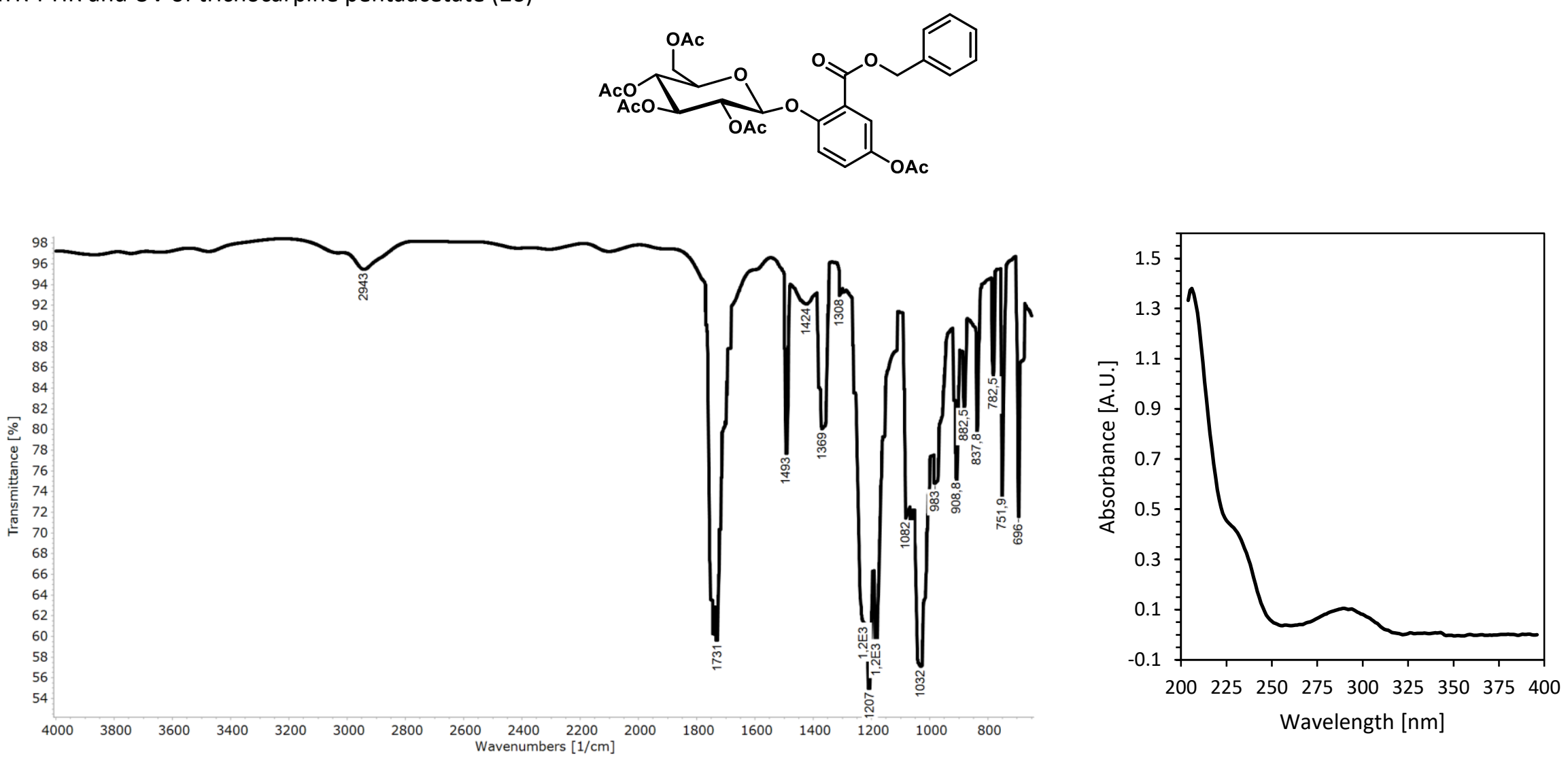
Trichoside tetraacetate (17)

${ }^{1} \mathrm{H}$ NMR (400 MHz, $\mathrm{CDCl}_{3}$ ) of trichoside tetraacetate (17)

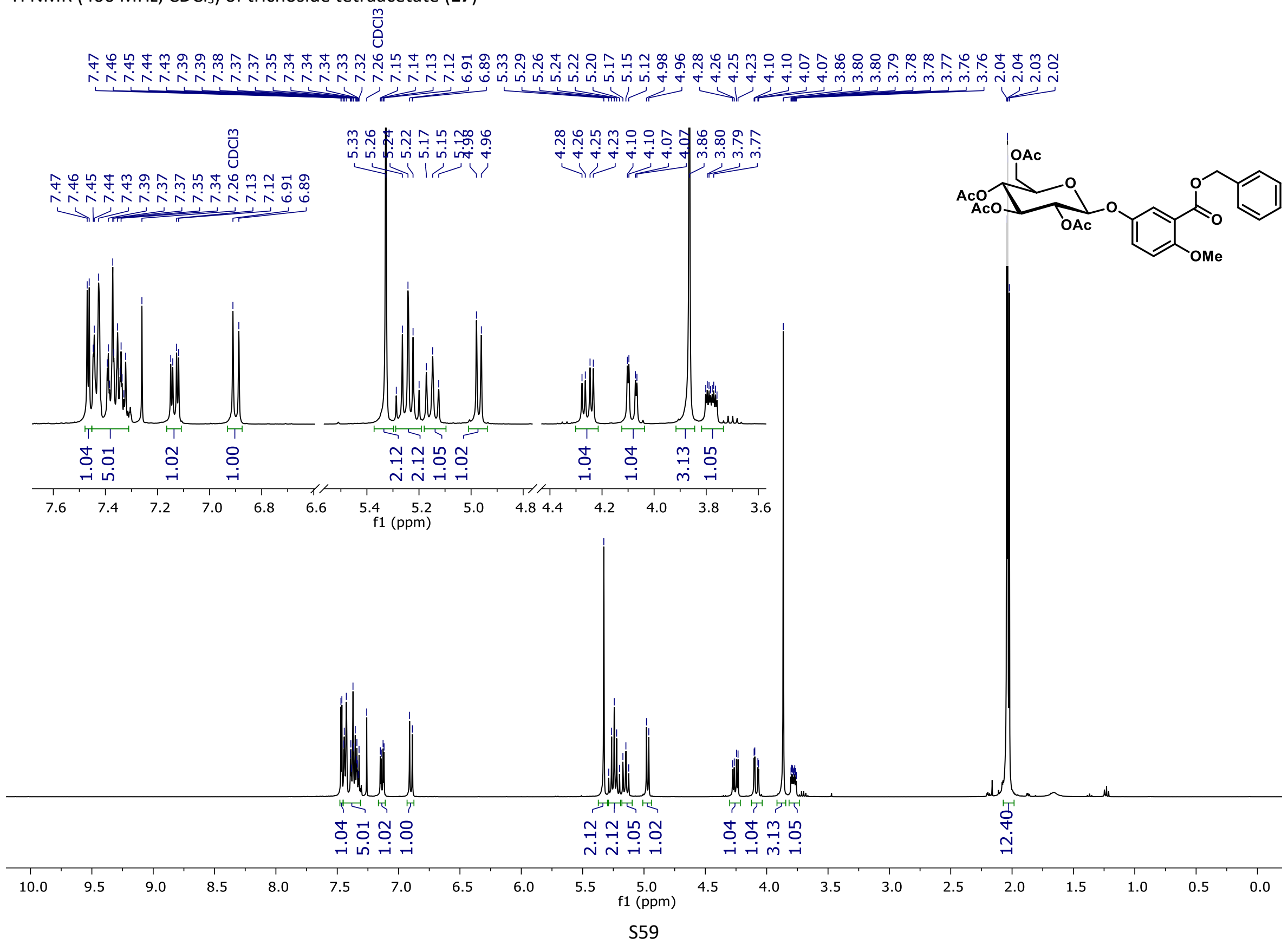


${ }^{13} \mathrm{C}$ NMR (101 MHz, $\mathrm{CDCl}_{3}$ ) of trichoside tetraacetate (17)
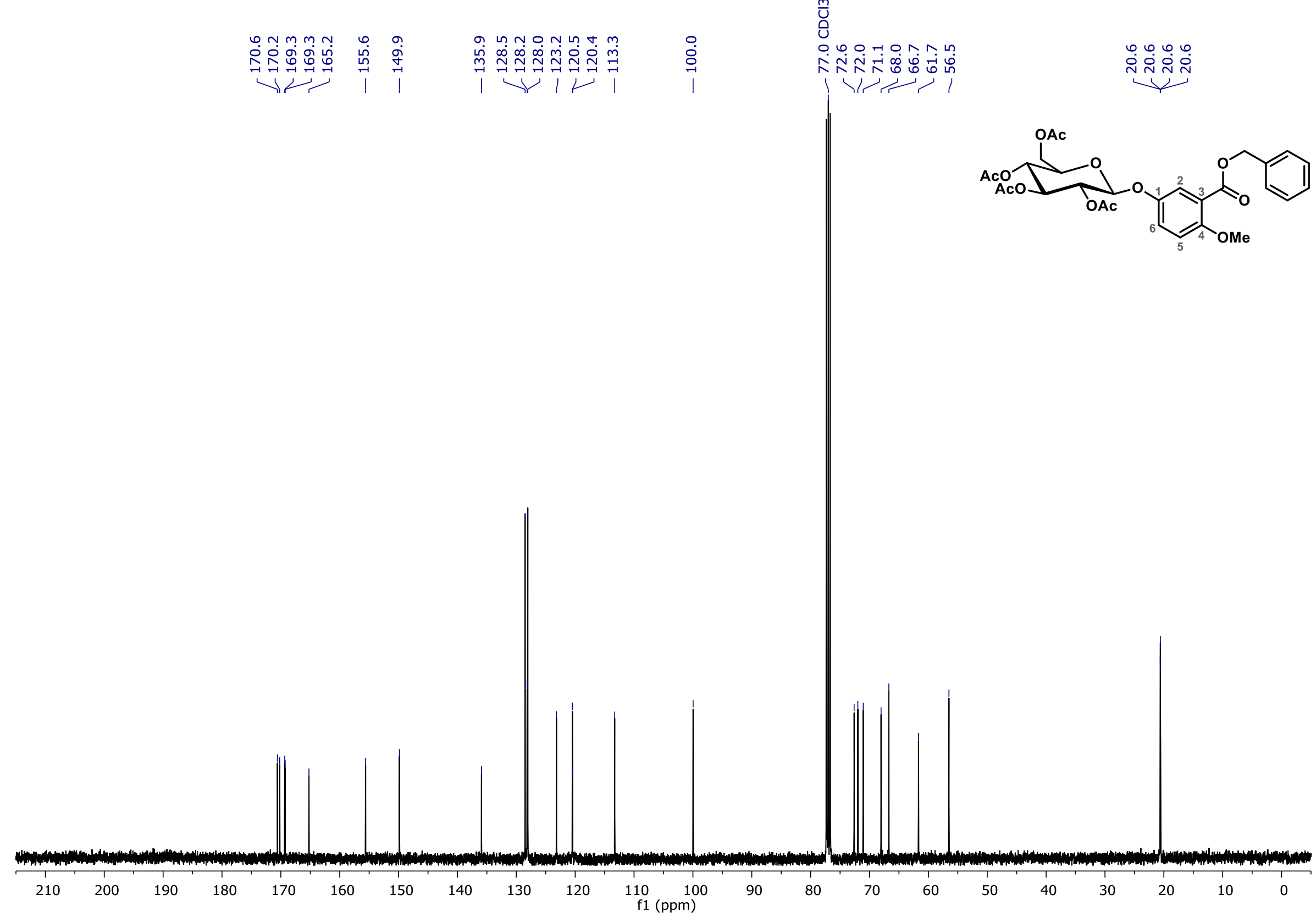


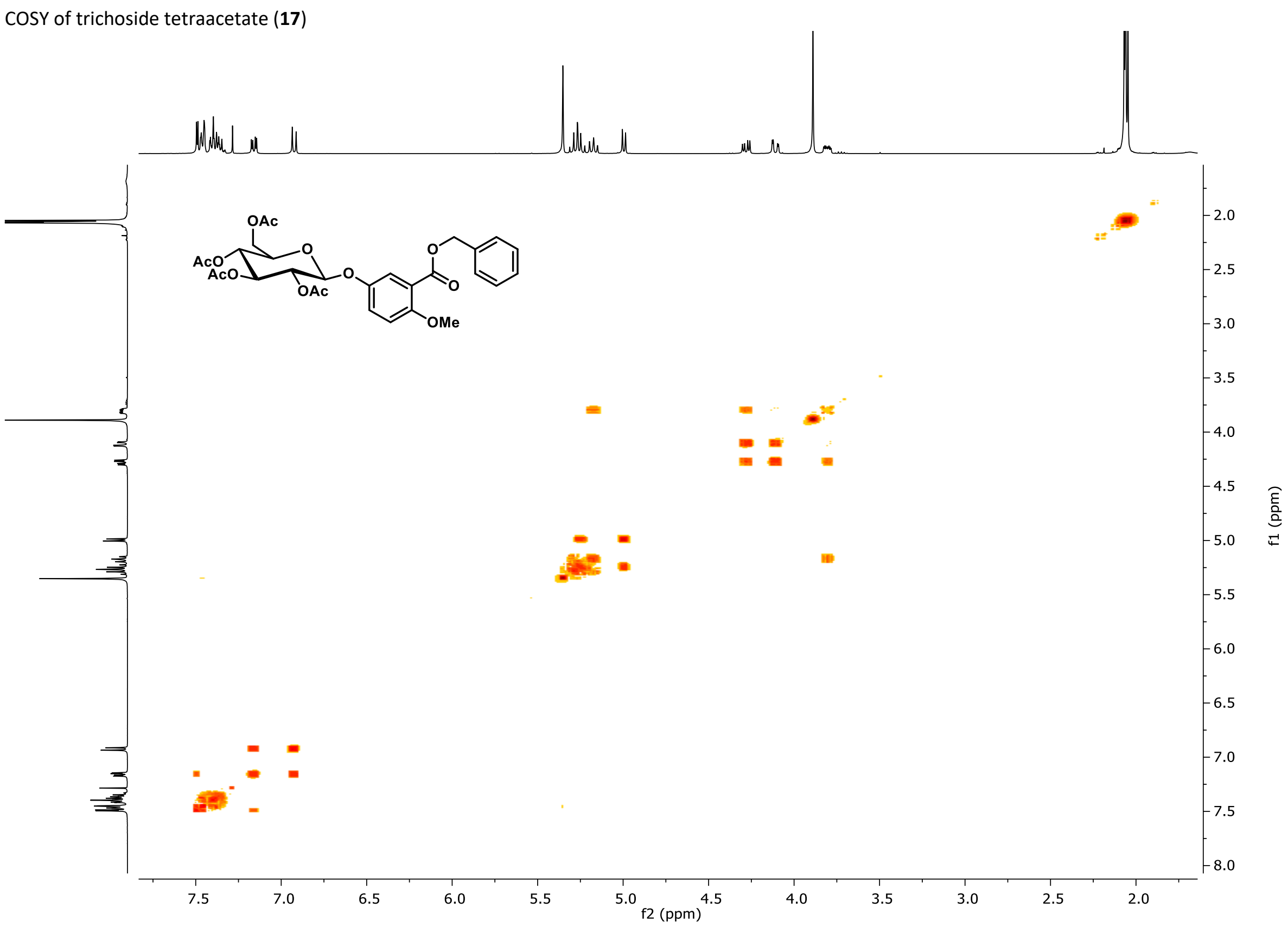


HSQC of trichoside tetraacetate (17)

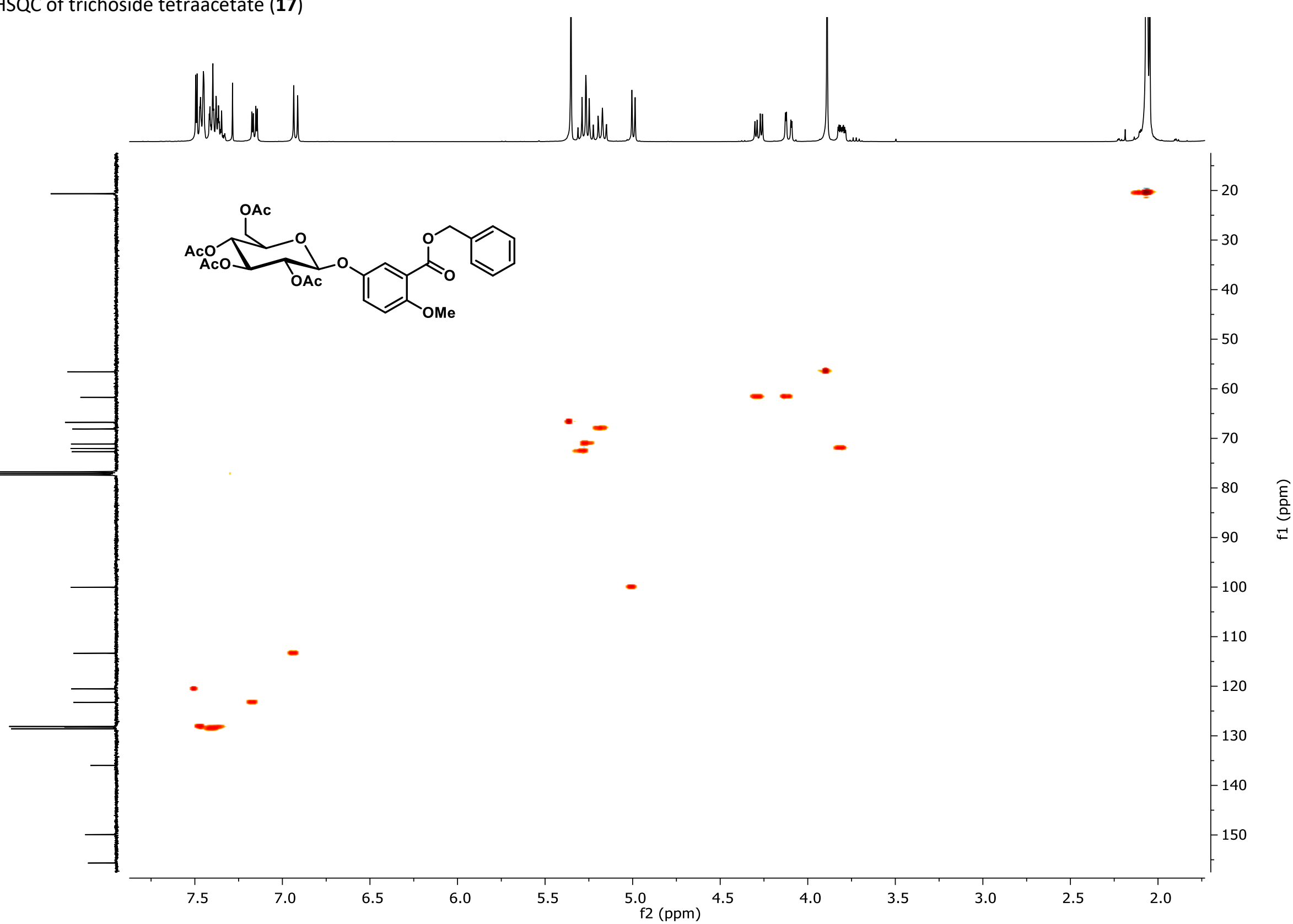


ATR-FTIR and UV of trichoside tetraacetate (17)
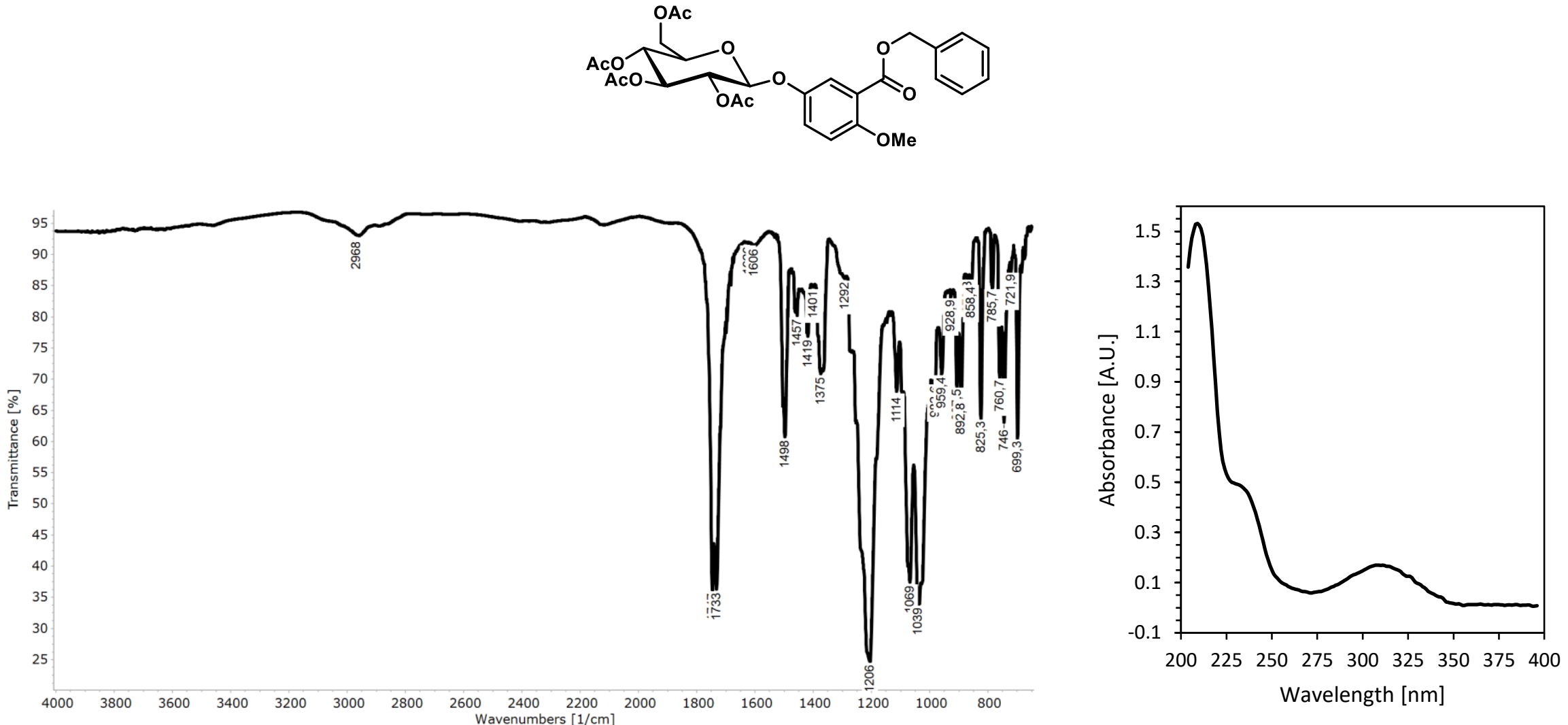


\section{Deoxytrichocarpine tetraacetate (18)}

${ }^{1} \mathrm{H}$ NMR (400 MHz, $\mathrm{CDCl}_{3}$ ) of deoxytrichocarpine tetraacetate (18)

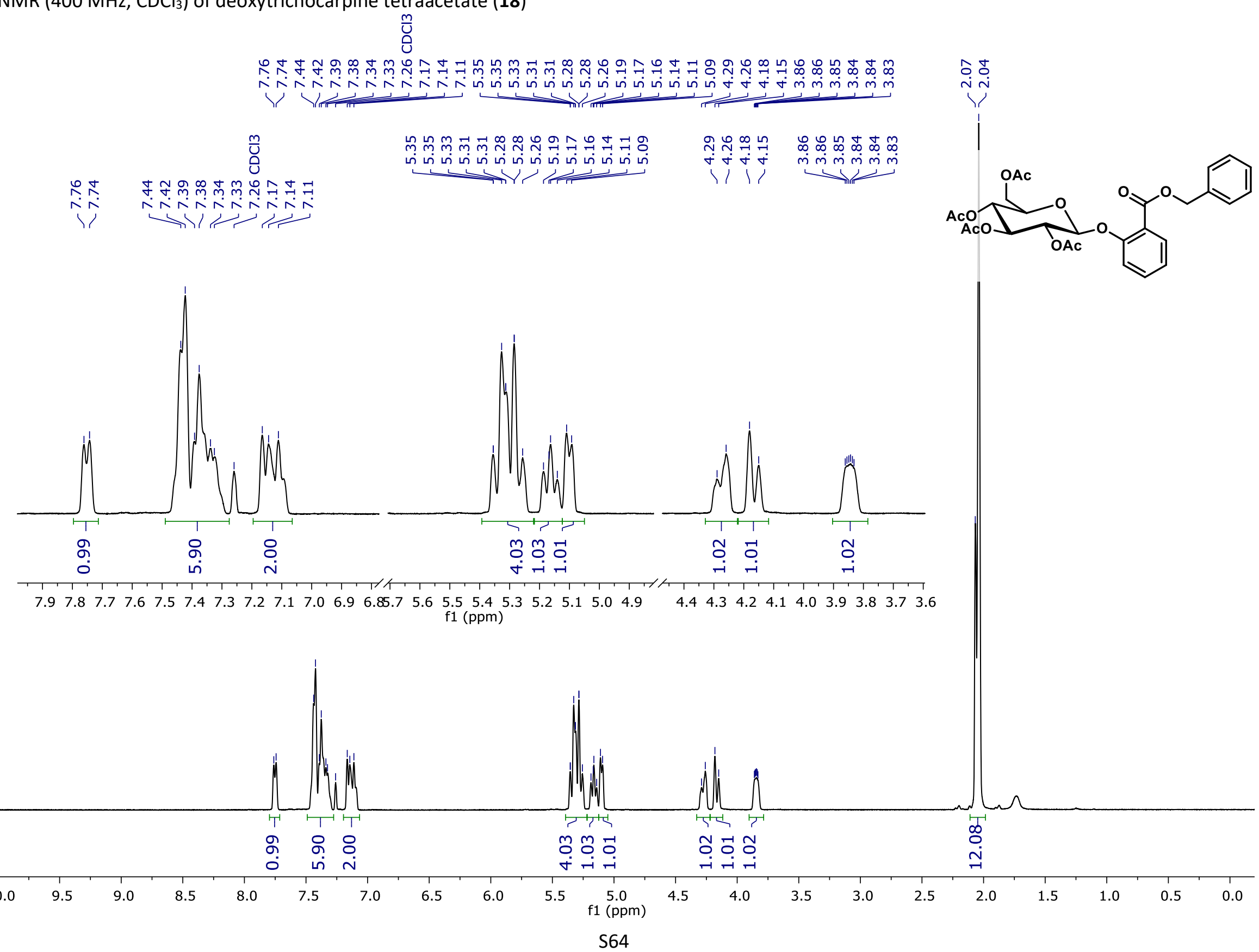


${ }^{13} \mathrm{C} \mathrm{NMR}\left(101 \mathrm{MHz}, \mathrm{CDCl}_{3}\right.$ ) of deoxytrichocarpine tetraacetate (18)

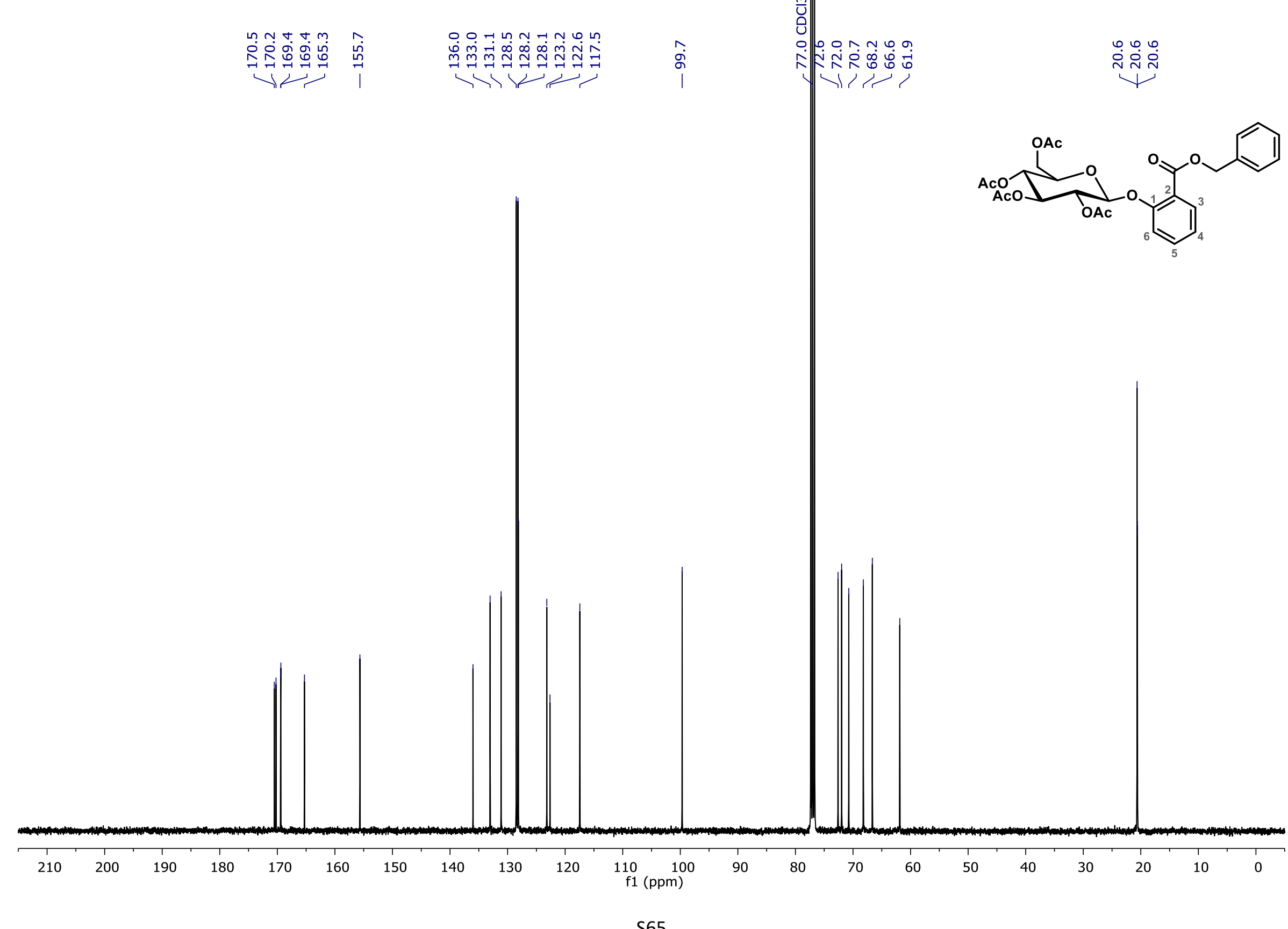


COSY of deoxytrichocarpine tetraacetate (18)

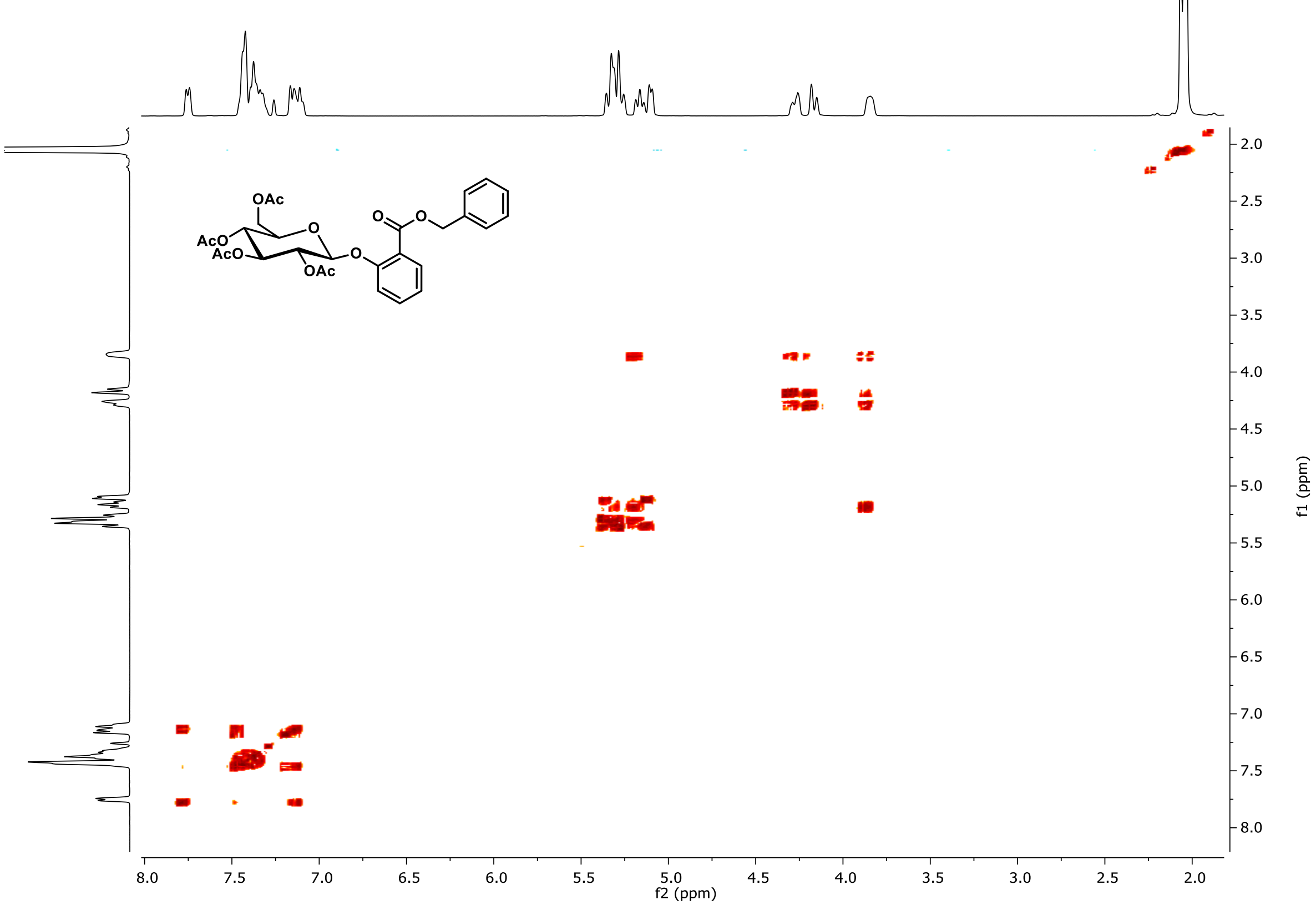


HSQC of deoxytrichocarpine tetraacetate (18)

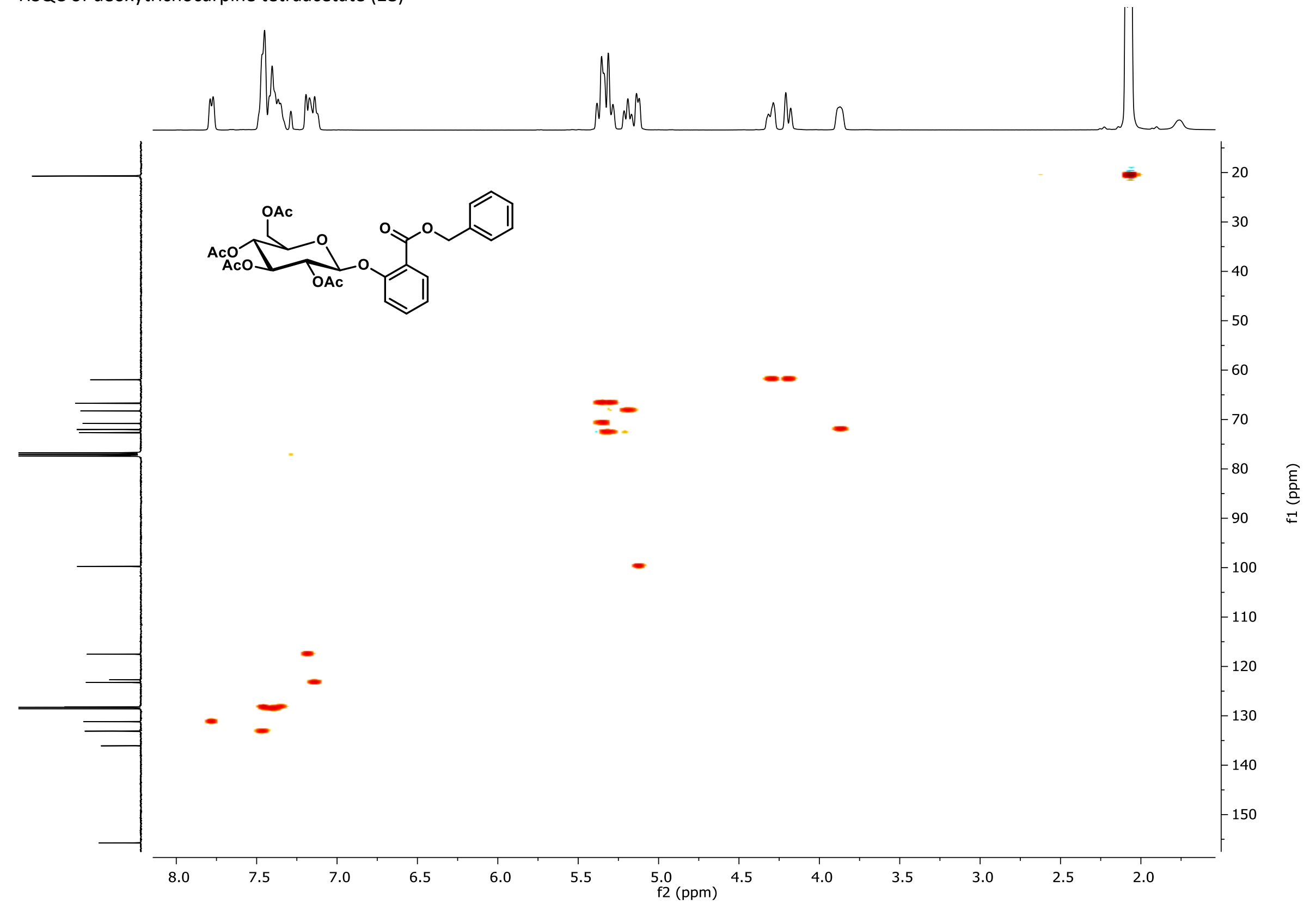


ATR-FTIR and UV of deoxytrichocarpine tetraacetate (18)
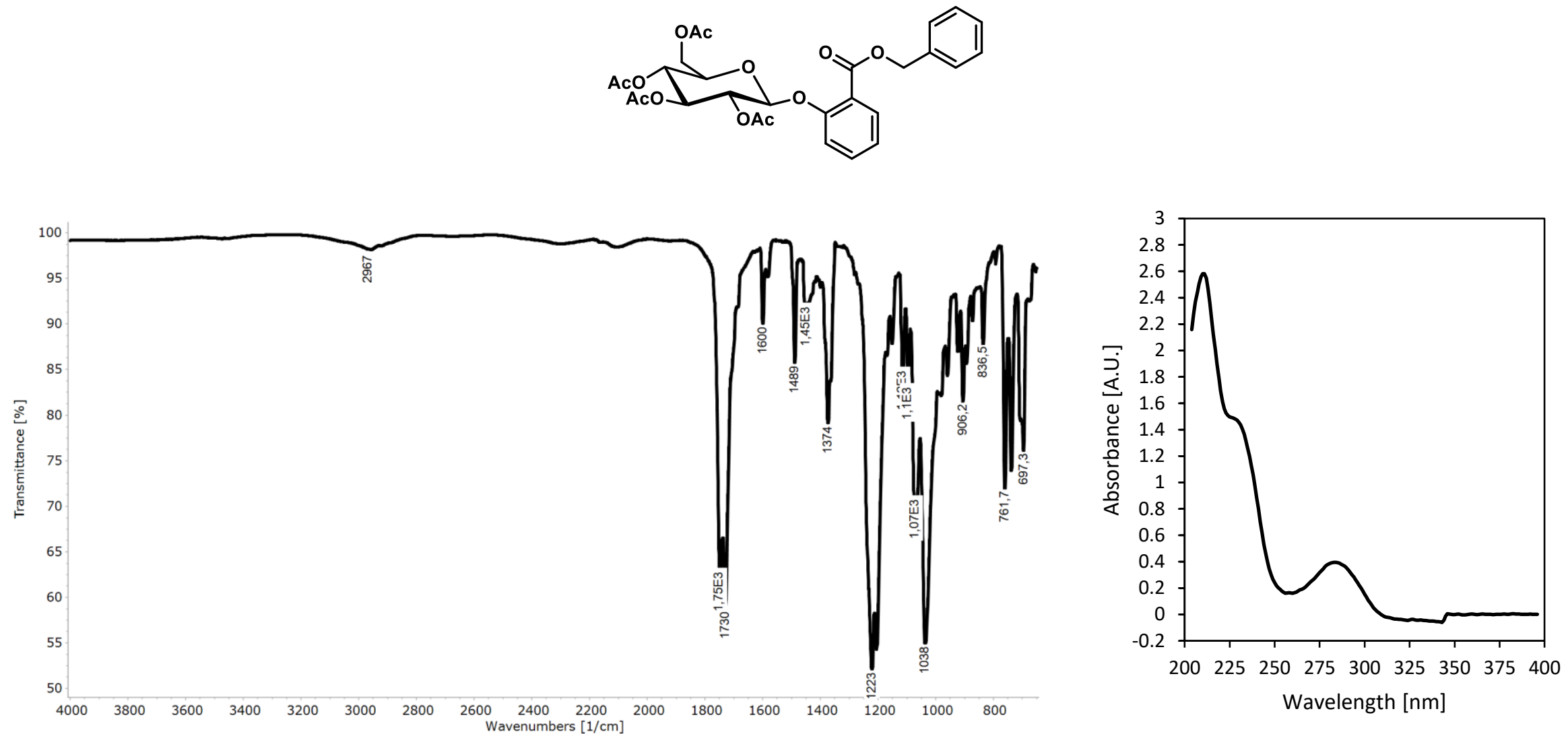


\section{2-O-Acetylisotrichocarpine (19)}

${ }^{1} \mathrm{H}$ NMR (400 MHz, DMSO- $d_{6}$ ) of 2-O-acetylisotrichocarpine (19)
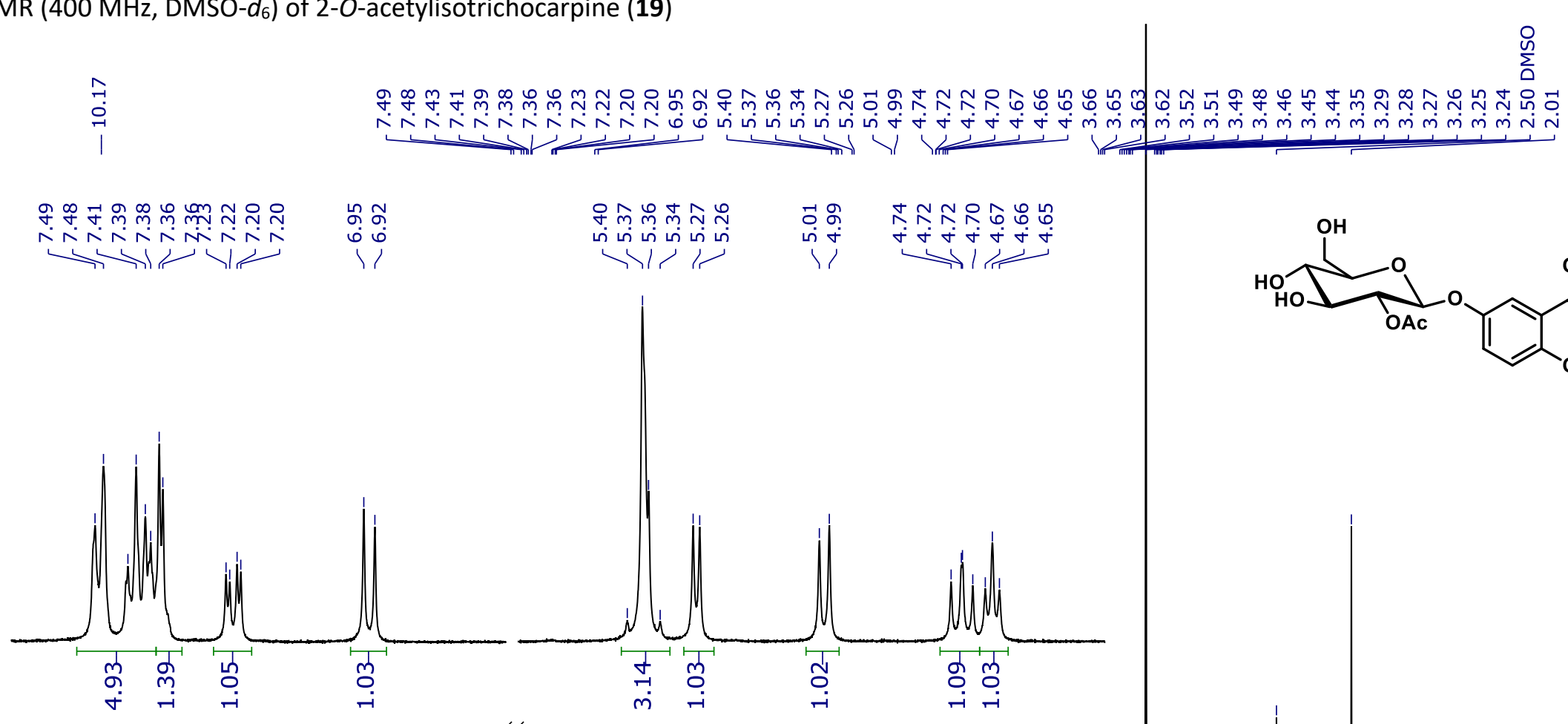

$\begin{array}{lllllllllllllllllllllll}7.6 & 7.5 & 7.4 & 7.3 & 7.2 & 7.1 & 7.0 & 6.9 & 6.8 & 6.7 & 5.6 & 5.5 & 5.4 & 5.3 & 5.2 & 5.1 & 5.0 & 4.9 & 4.8 & 4.7 & 4.6 & 4.5\end{array}$

f1 (ppm)

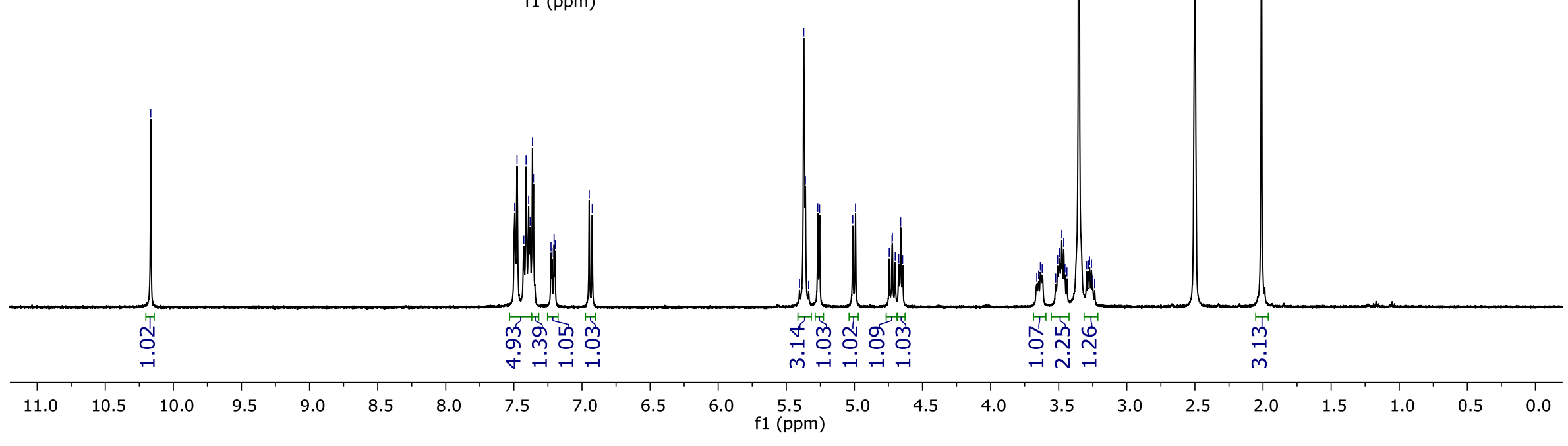


${ }^{13} \mathrm{C}$ NMR (101 MHz, DMSO- $d_{6}$ ) of 2-O-acetylisotrichocarpine (19)

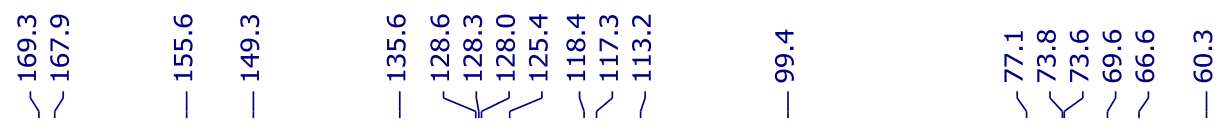
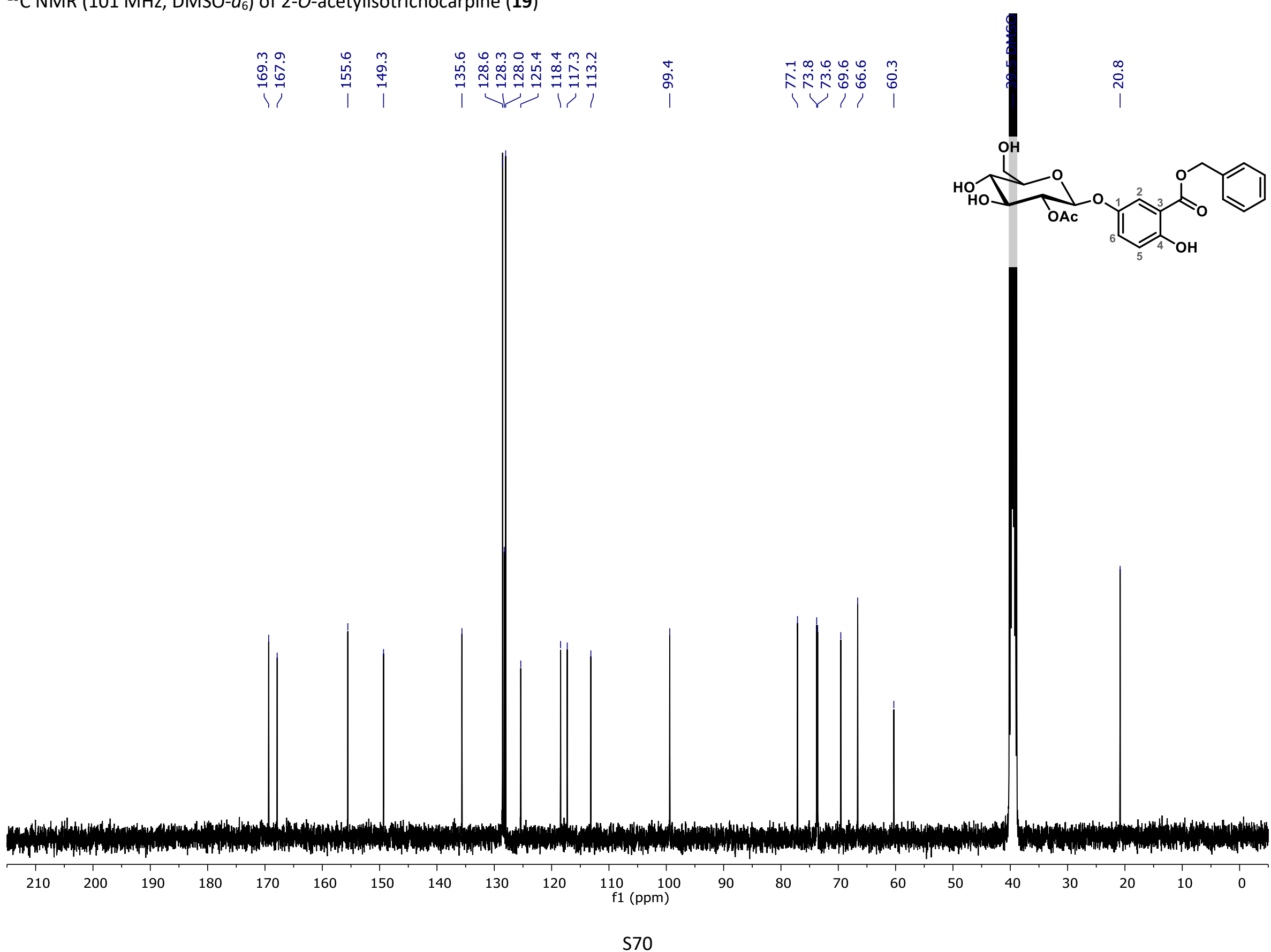
COSY of 2-O-acetylisotrichocarpine (19)

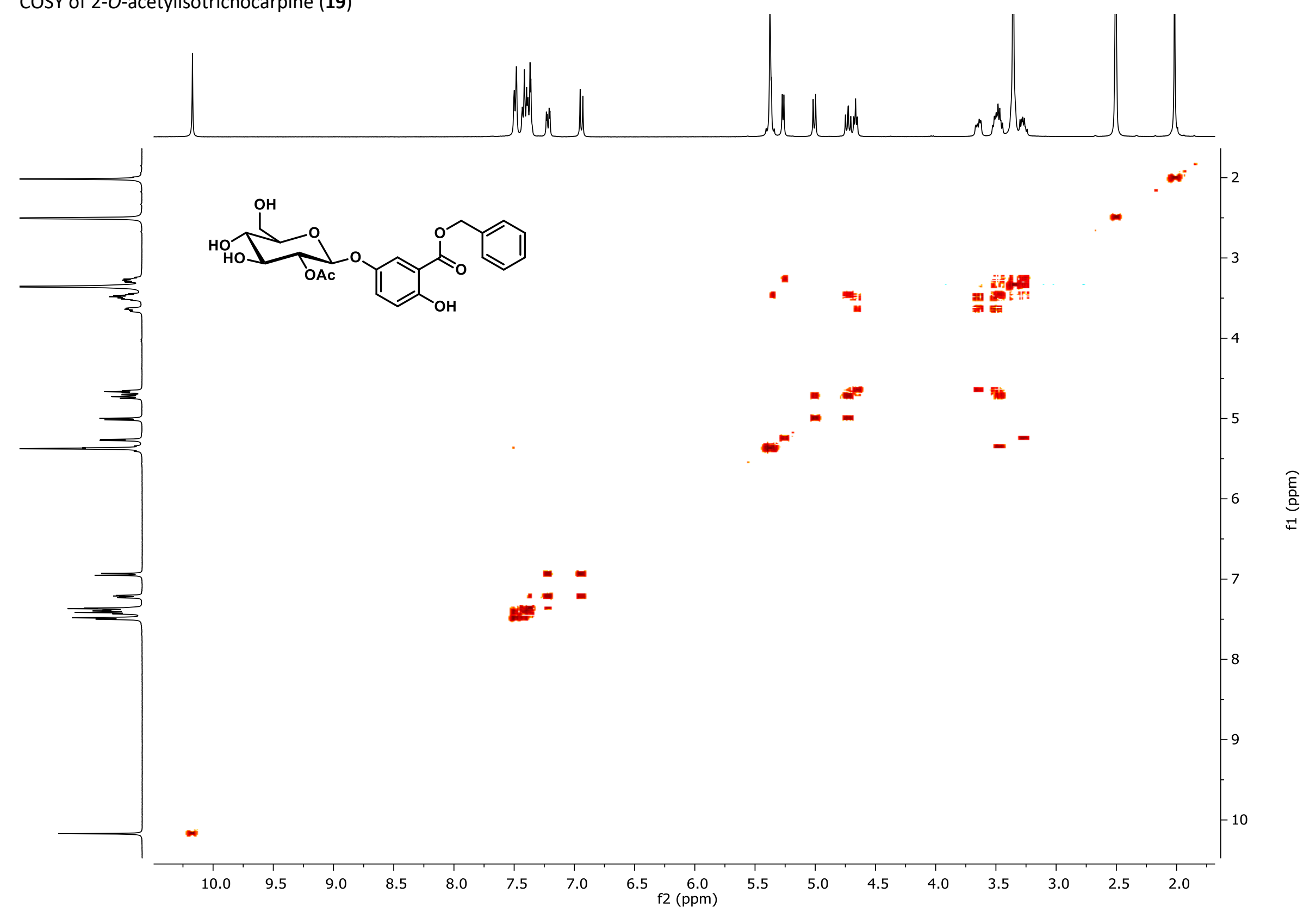


HSQC of 2-O-acetylisotrichocarpine (19)

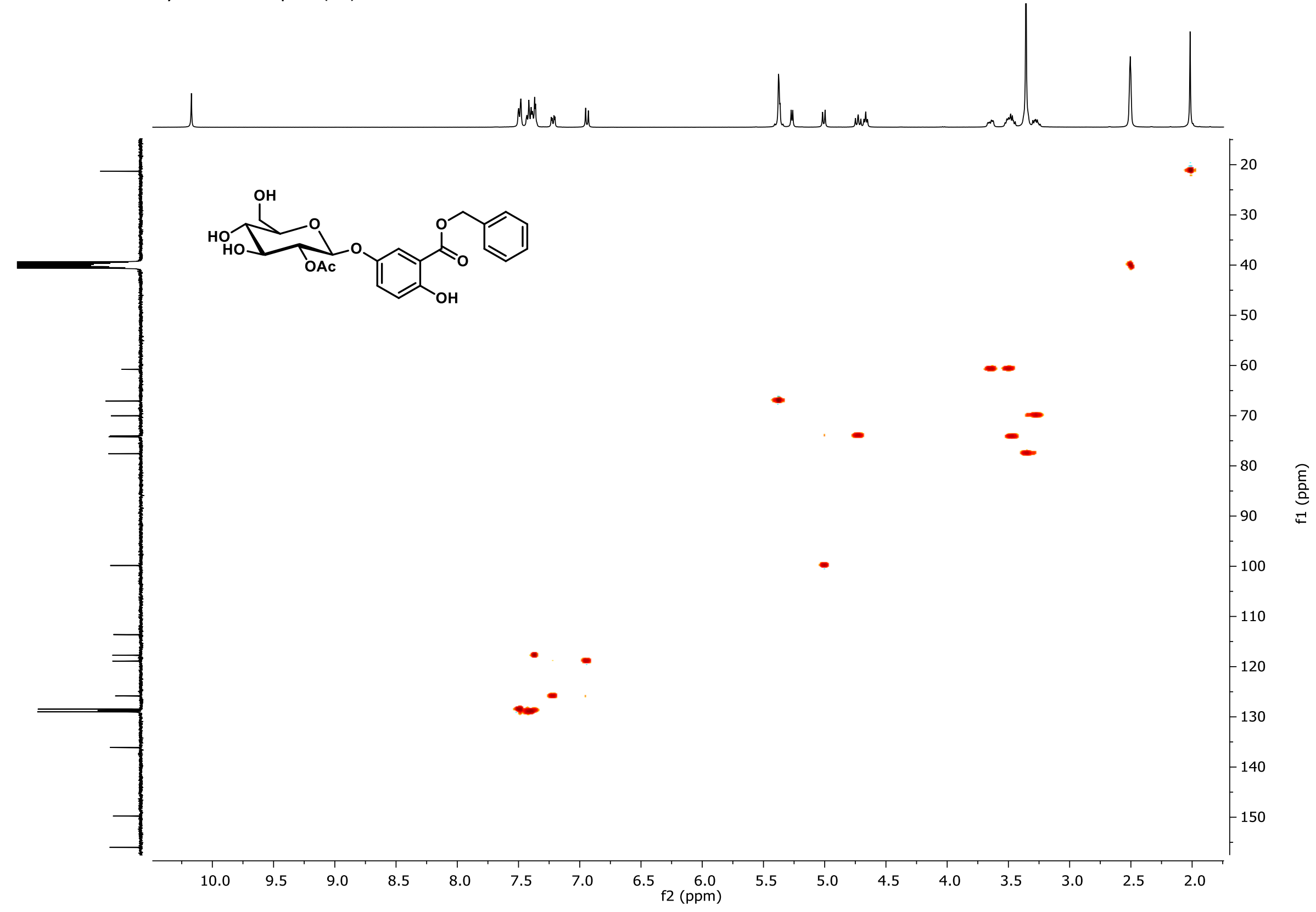



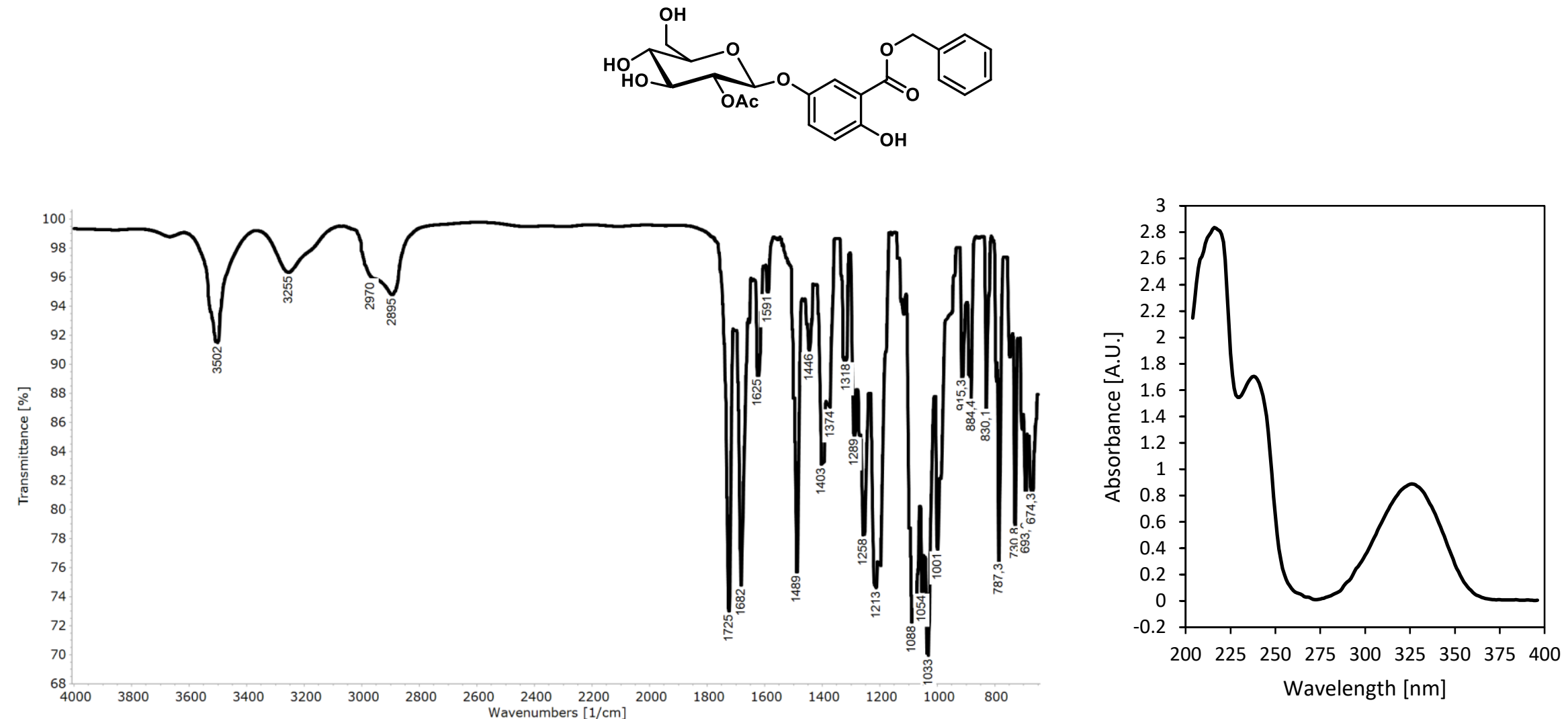


\section{2-O-Acetyltrichoside (20)}

${ }^{1} \mathrm{H}$ NMR (400 MHz, DMSO- $d_{6}$ ) of 2-O-acetyltrichoside (20)

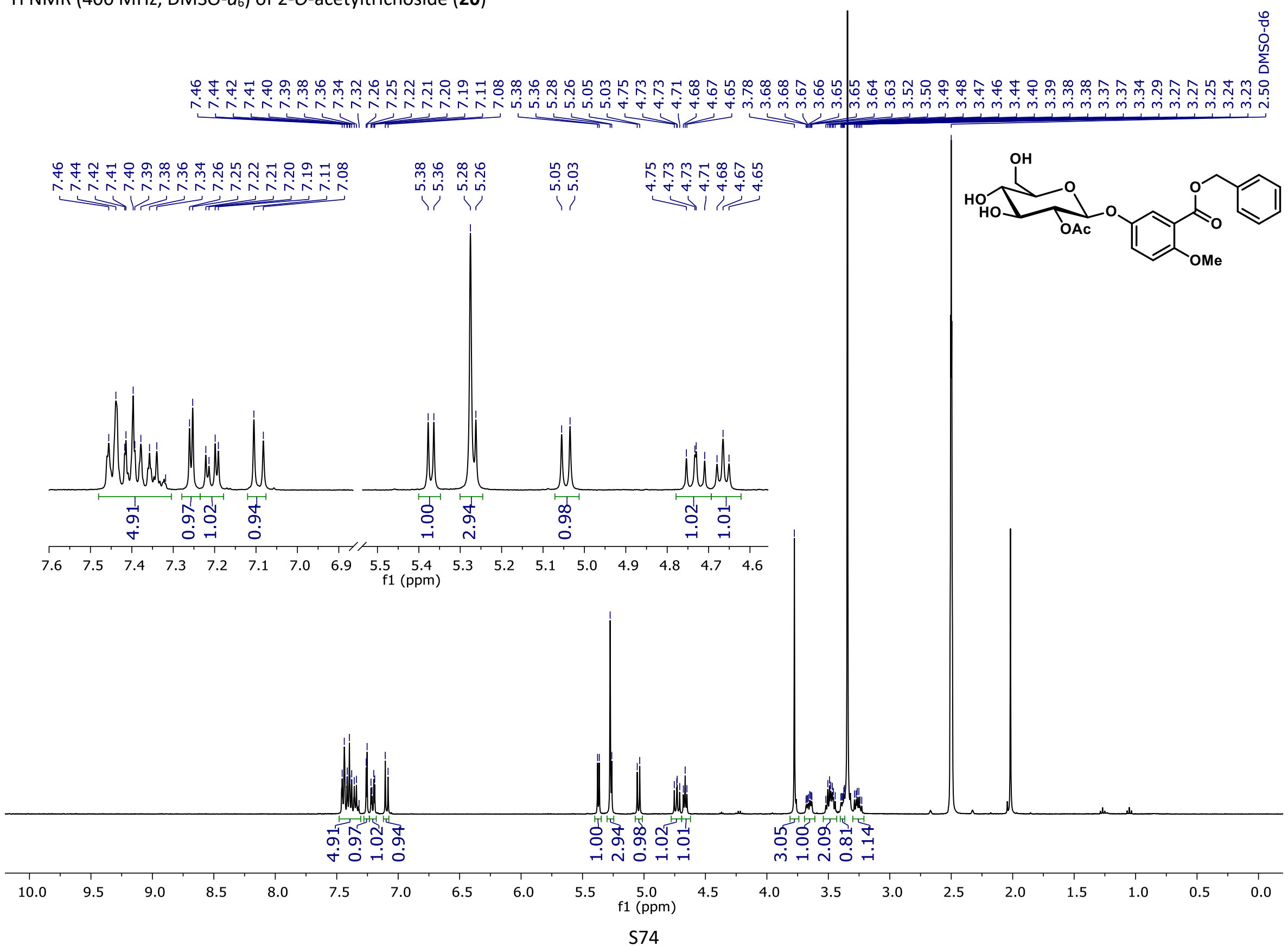



COSY of 2-O-acetyltrichoside (20)

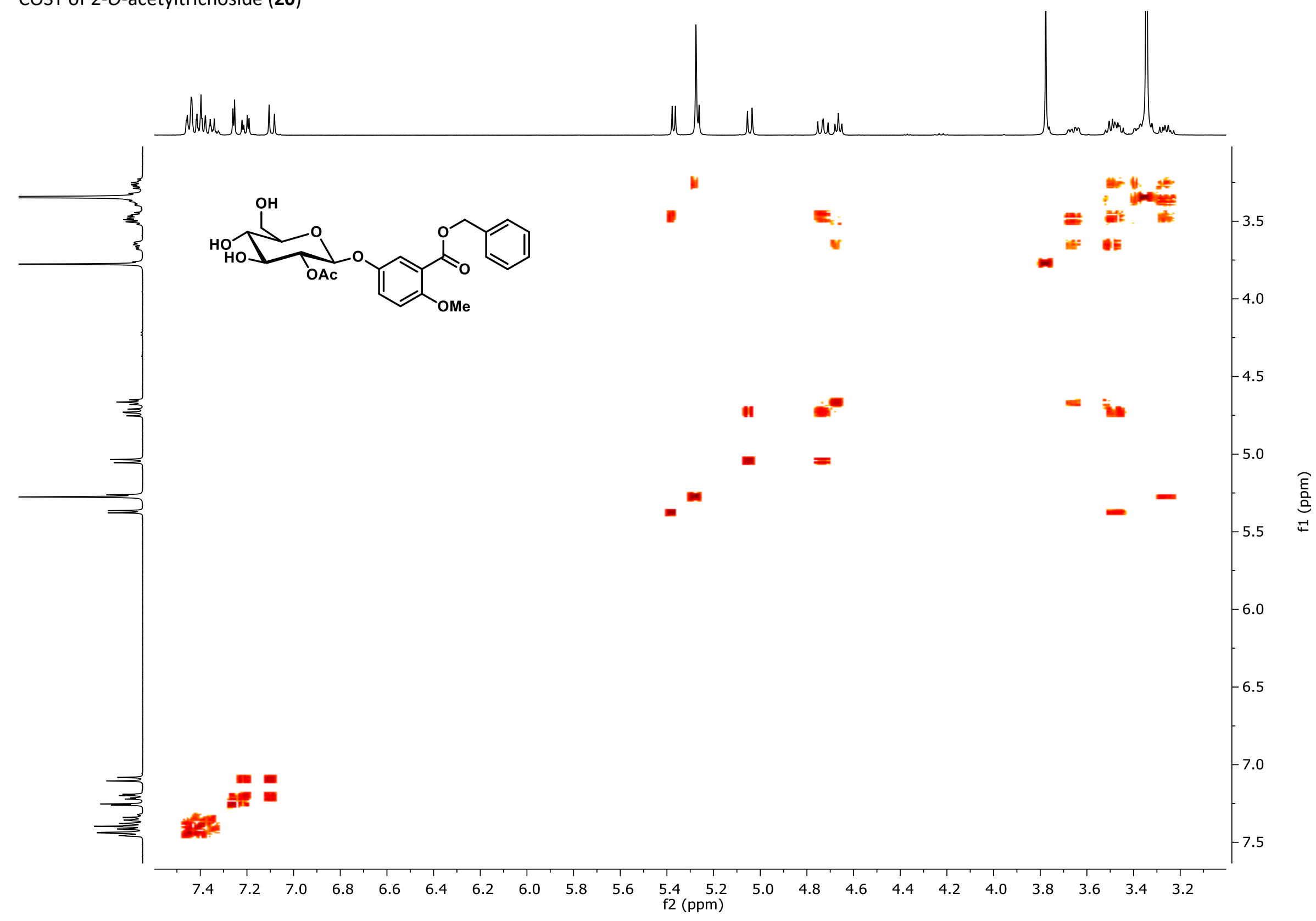


HSQC of 2-O-acetyltrichoside (20)

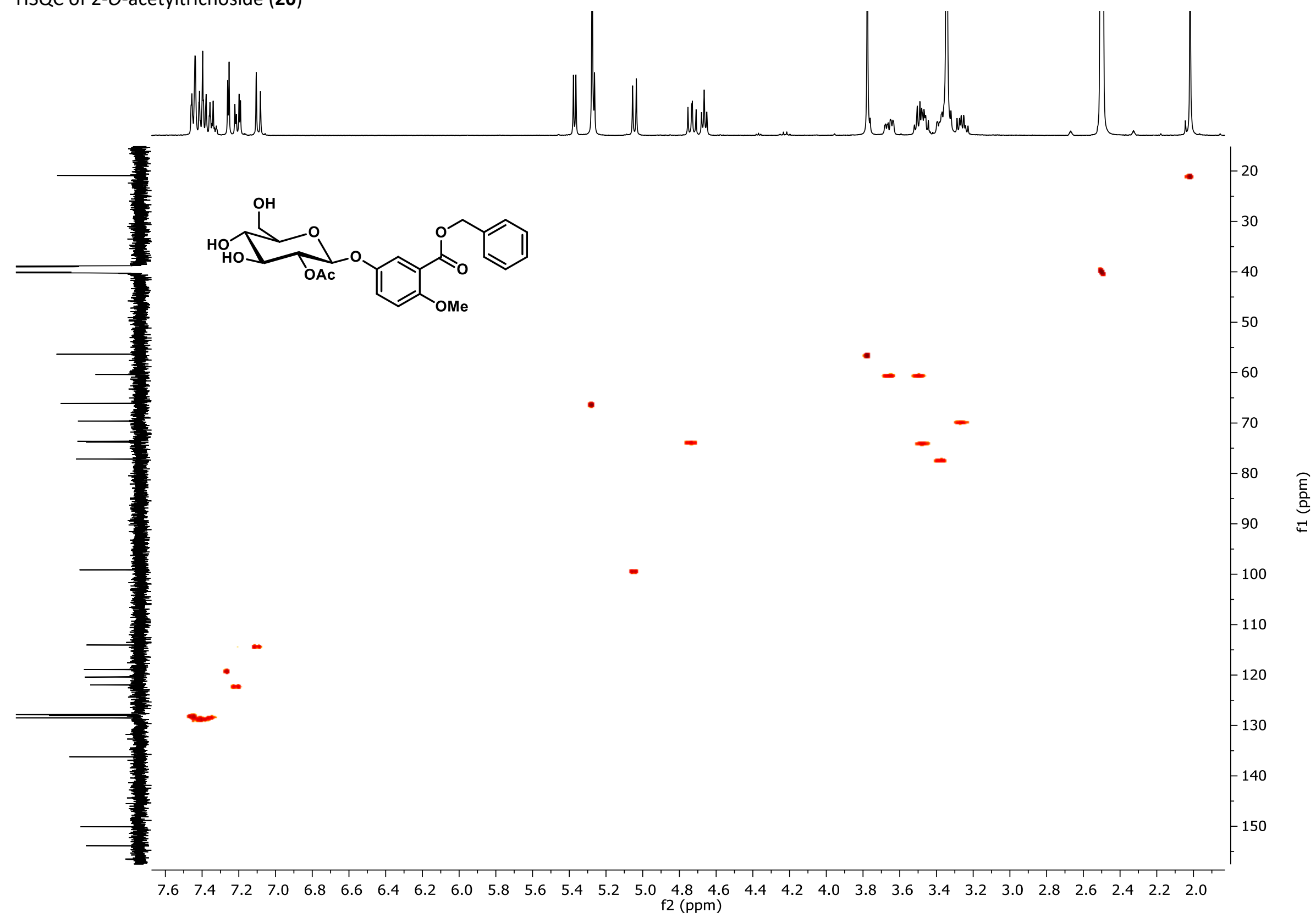



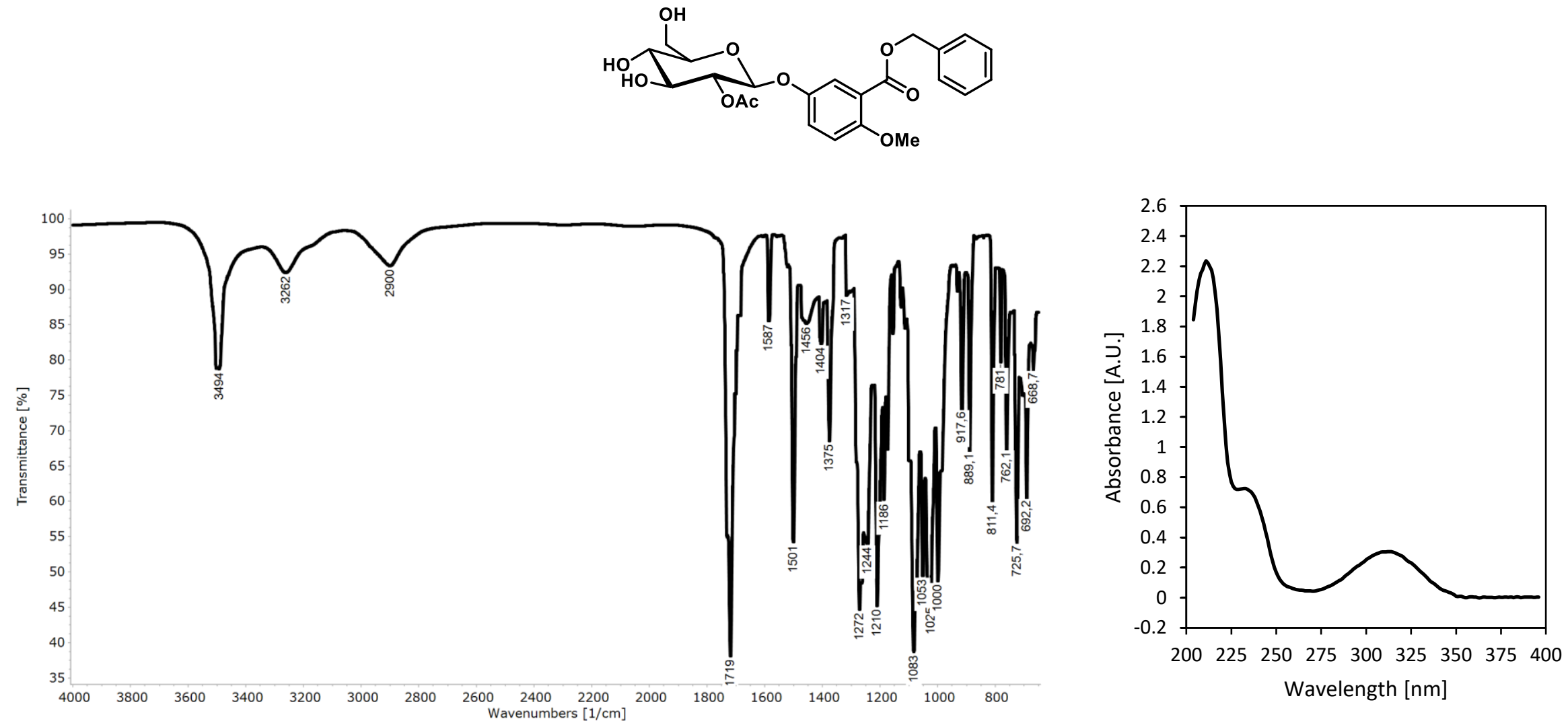


\section{2-O-Acetyldeoxytrichocarpine (21)}

${ }^{1} \mathrm{H}$ NMR (400 MHz, MeOD) of 2-O-acetyldeoxytrichocarpine (21)

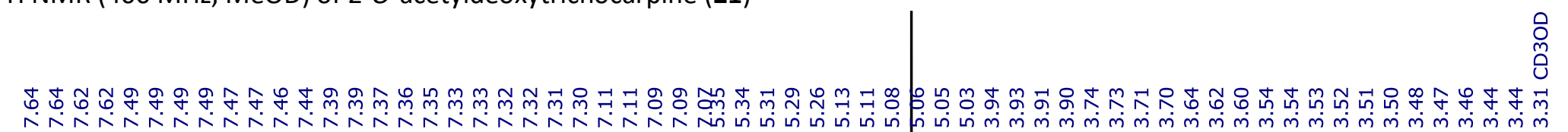

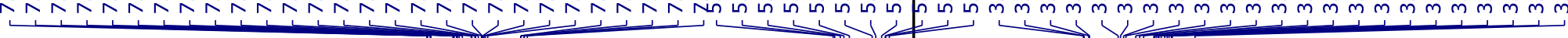

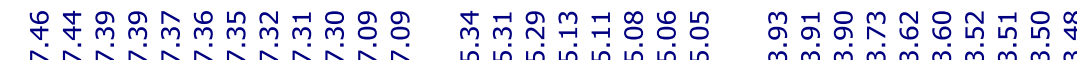
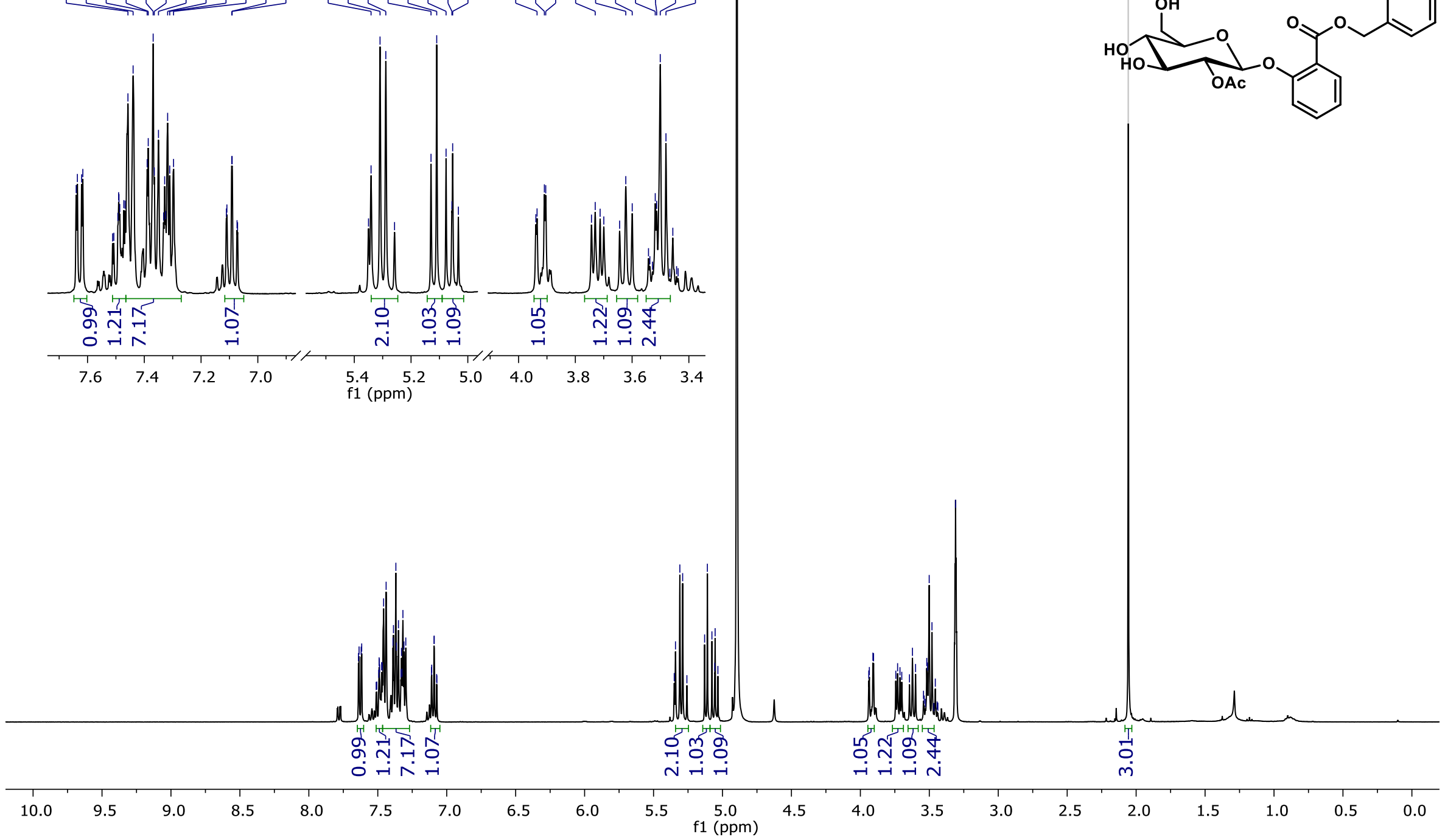
${ }^{13} \mathrm{C}$ NMR (101 MHz, MeOD) of 2-O-acetyldeoxytrichocarpine (21)

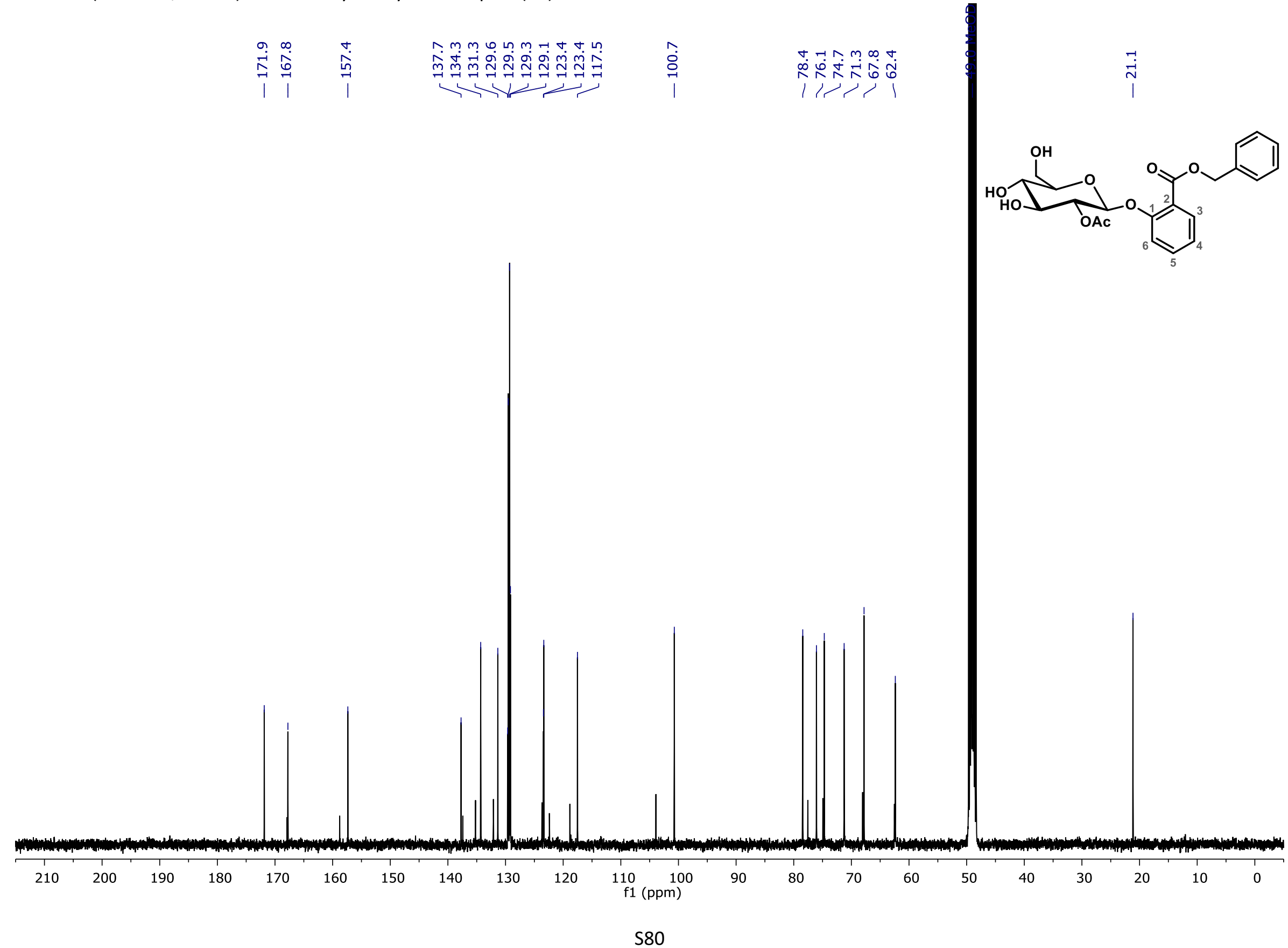


COSY of 2-O-acetyldeoxytrichocarpine (21)

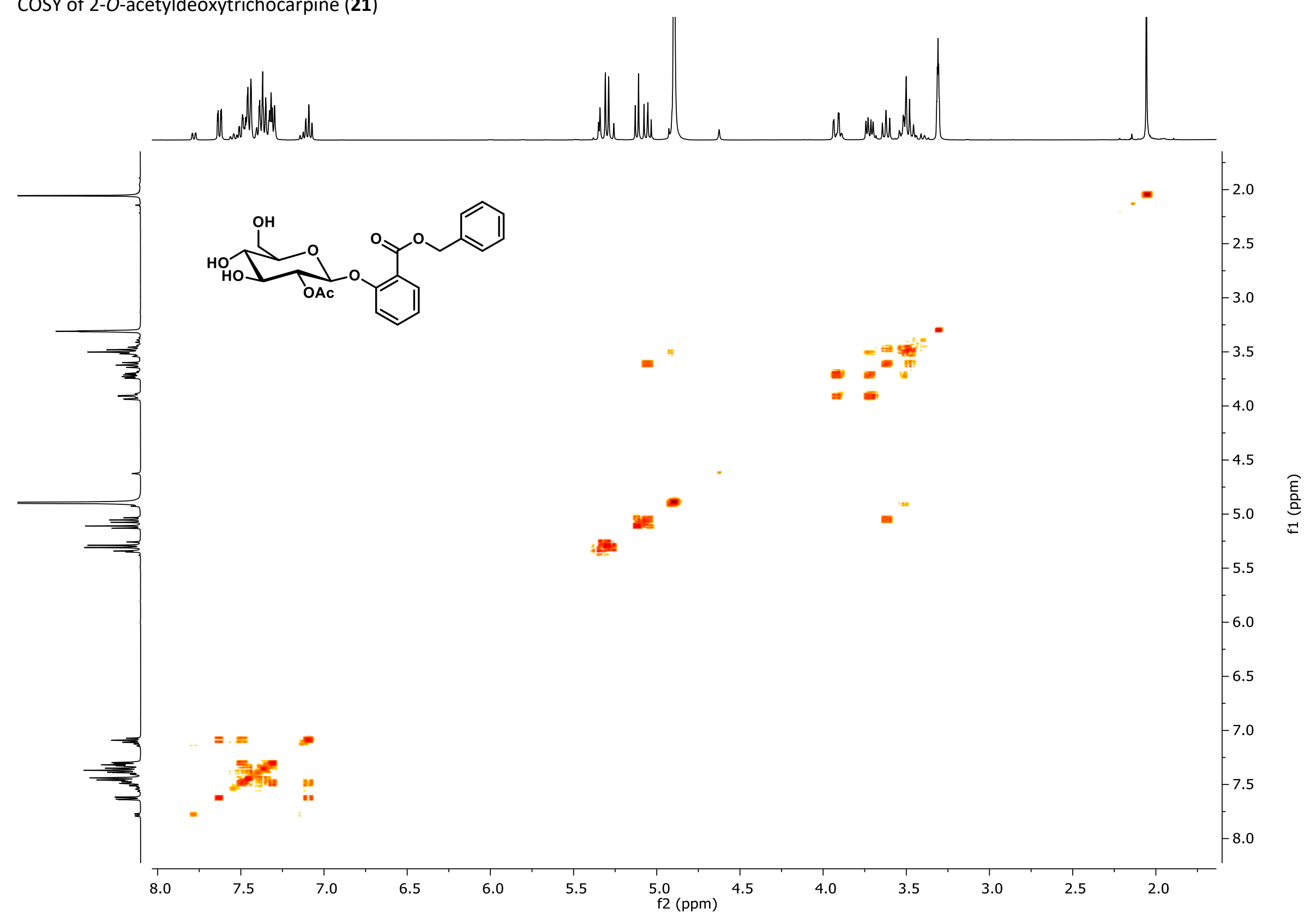


HSQC of 2-O-acetyldeoxytrichocarpine (21)

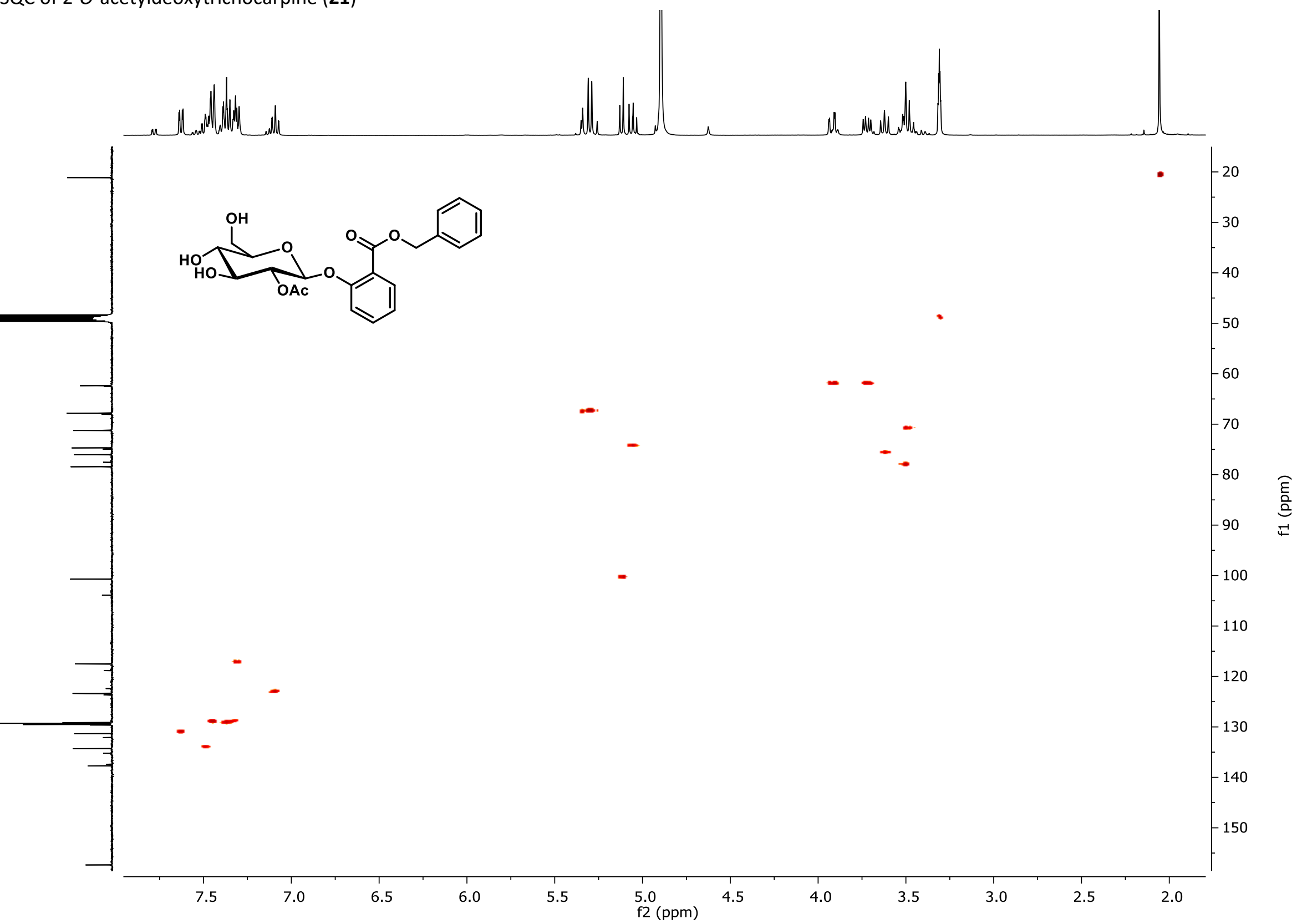



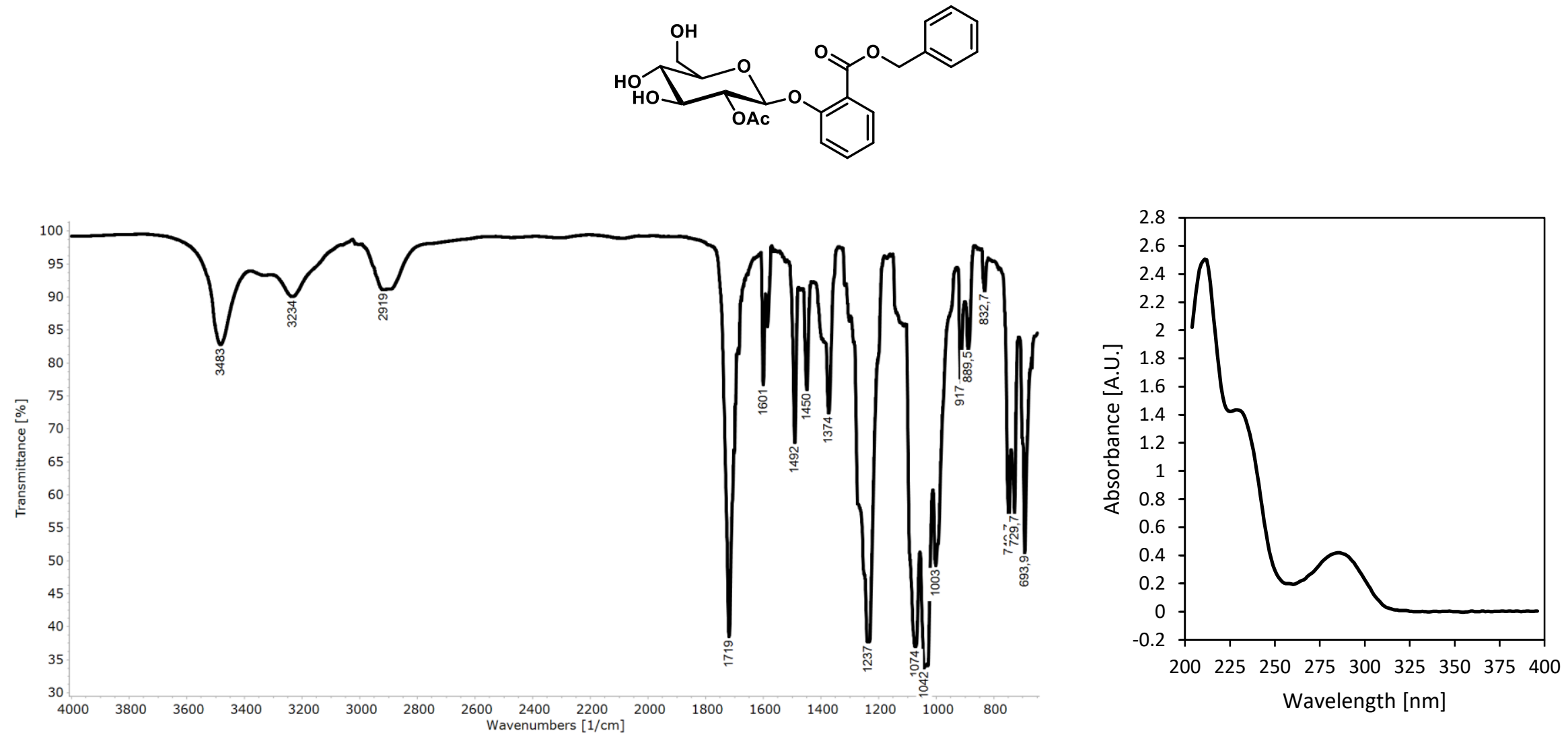